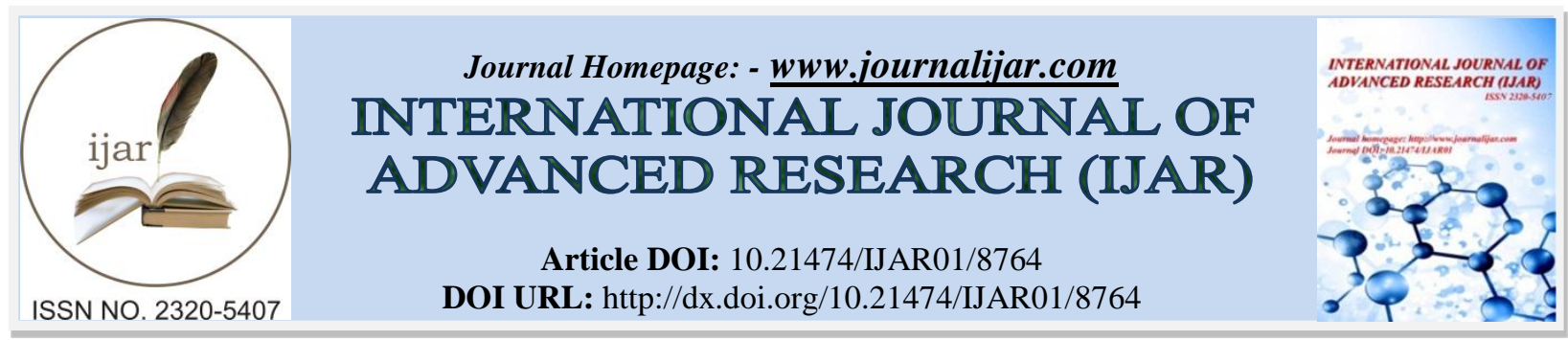

RESEARCH ARTICLE

\title{
INJURIES WITH ALL TYPES OF ANESTHESIA.
}

Dr. Sherif Atef Gomaa Ali ${ }^{1}$ and Dr. Amr Ahmed Abdelrahman Abdelwahed ${ }^{2}$.

1. M.B.B.CH, MSC Anesthesia, F.E.B Anesthesia, Anesthesia department, Hatta Hospital, Dubai, Health Authority.

2. M.B.B.CH, MSC Anesthesia, Anesthesia department, Hatta Hospital, Dubai, Health Authority.

\section{Manuscript Info}

Manuscript History

Received: 20 January 2019

Final Accepted: 22 February 2019

Published: March 2019

\section{Abstract}

Copy Right, IJAR, 2019,. All rights reserved.

\section{Introduction:-}

The anaesthetized patient is at risk of complications resulting from the actions, or inactions, of the anesthetist, from the actions of the surgeon, and from failure or malfunction of anesthetic equipments. The state of anesthesia may be considered to be intrinsically unsafe. Unconsciousness carries with it risks of airway obstruction, soiling of the lungs, and inability to detect peripheral nerve injury (Aitkenhead, 2005).

Injury to airway structures is a constant concern to practicing anesthesiologists. The daily insertion of endotracheal tubes, laryngeal mask airways, oral/nasal airways, gastric tubes, transesophageal echocardiogram (TEE) probes, esophageal (boogie) dilators, and emergency airways all involve the risk of airway structure damage. Common morbidities such as sore throat and dysphagia are usually self-limiting but may also be nonspecific symptoms of more ominous complications (Morgan, 2005).

Perioperative nerve injury is a known complication of both regional and general anesthesia. Peripheral nerve injury is often a debilitating problem. In most cases, these injuries resolve within 6-12 weeks, but some persist for months or even years. Because peripheral neuropathies are commonly associated (sometimes incorrectly!) with patient positioning, a review of mechanisms and prevention is necessary (Morgan, 2005).

Neurological complications related to regional anesthesia vary from parethesia up to spinal cord injury. Direct trauma to nervous system may occur at the level of the spinal cord , nerve root, or peripheral nerve (Auroy et al., 1997).

Neurologic complications associated with spinal or epidural anesthesia can be due to toxic effects of the injected agent, incorrect placement of a needle or catheter causing direct neural tissue damage, infectious agents, or spinal cord compromise due to ischemia or mass effect. Adverse events related to the surgical procedure, positioning, or a patient's underlying medical condition can also present as "complications" of regional anesthesia. Anticipation and prevention of complications, along with their early diagnosis and treatment are the most important factors in dealing with regional anesthetic risks. Several large studies have confirmed the rarity of permanent neurologic injury associated with this type of anesthesia (Wedel, 2000).

Corresponding Author:- Sherif Atef Gomaa Ali.

Address:-M.B.B.CH, MSC Anesthesia, F.E.B Anesthesia, Anesthesia department, Hatta Hospital, Dubai, Health Authority. 
There are relatively few published reports of complications associated with the use of peripheral nerve blocks. Because there is a relative paucity of published information on the mechanisms of neuronal injury after nerve blockade and methods to prevent them (Stan et al, 1995).

In general, distal peripheral blocks are associated with a lower risk of complications. However, intravascular injection can occur, and the usual precautions of incremental injection after aspiration are recommended. The risk of nerve injury is theorized to be higher when more distal peripheral blocks are performed, possibly because of superficial nerve placement between bony and ligamentous structures, thereby offering ready access to the probing needle point (Miller 2005).

\section{Injuries associated with General Anesthesia}

Many injuries sustained during anesthesia are due to human error and may be avoided through high standards of clinical practice. Dental injury occurs during $1 \%$ of general anesthetics and is the commonest cause for litigation against anesthetists. Other injuries can include other injuries such as, ventilation related injuries and ophthalmic injuries. Injuries related to positioning and cannulation can occur with any anesthetics techniques (Morgan et al., 2006).

\section{Anatomy Of The Airway}

There are two openings to the human airway: the nose, which leads to the nasopharynx, and the mouth, which leads to the oropharynx. The pharynx is a U-shaped fibromuscular structure that extends from the base of the skull to the cricoid cartilage at the entrance to the esophagus. It opens anteriorly into the nasal cavity, the mouth and the larynx. At the base of the tongue, the epiglottis functionally separates the oropharynx from the laryngopharynx (or hypopharynx). The epiglottis prevents aspiration by covering the glottis - the opening of the larynx-during swallowing. The larynx is a cartilaginous skeleton held together by ligaments and muscle. The larynx is composed of nine cartilages thyroid, cricoid, epiglottic, and (in pairs) arytenoid, corniculate, and cuneiform (Morgan et al., 2006).

Figure 1-1 :

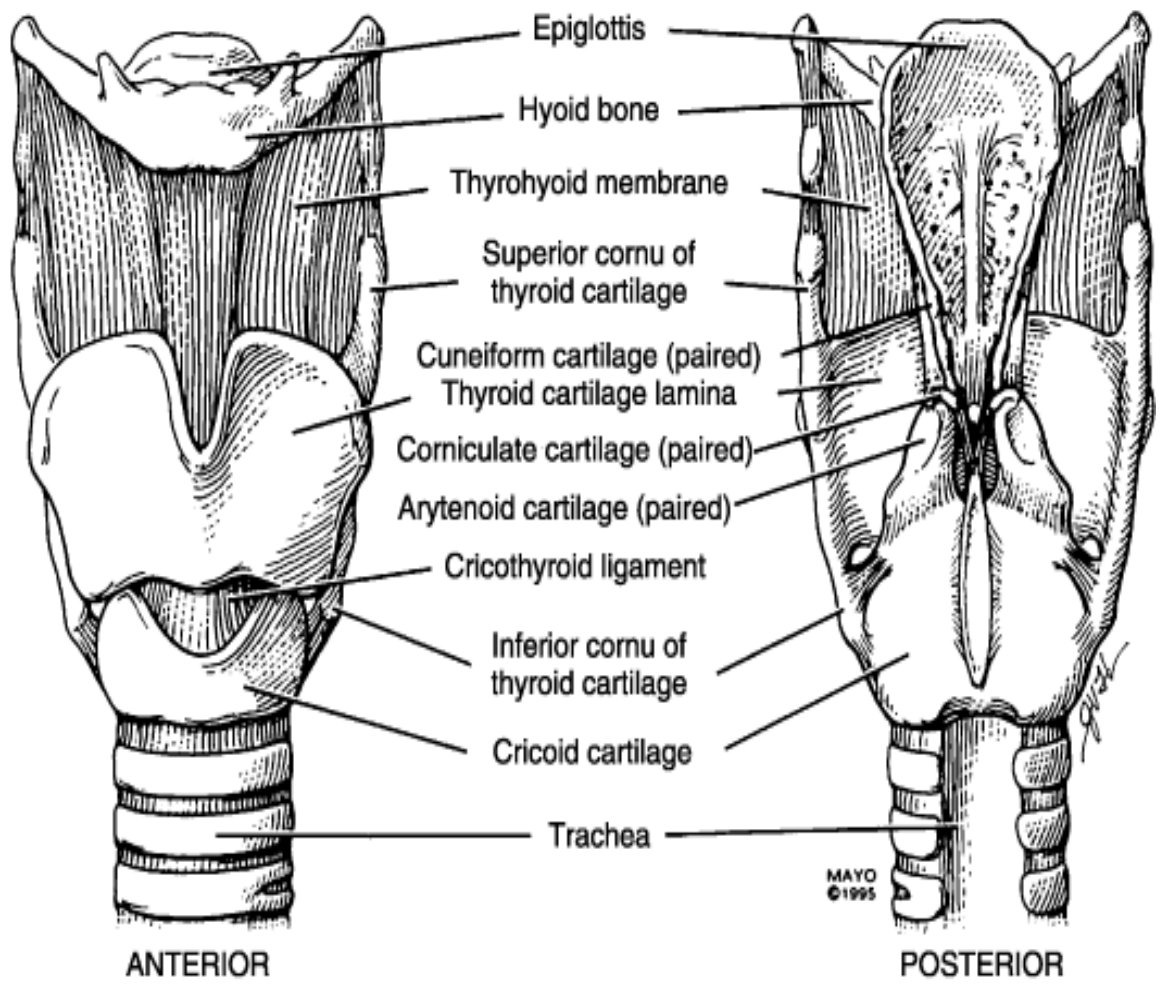

Cartilaginous structures of the larynx (In: Critical Care Medicine: Perioperative Management. Murray MJ, Coursin DB, Pearl RG, Prough DS [editors]. Lippincott-Raven Publishers, 1997.) 
The blood supply of the larynx is derived from branches of the thyroid arteries. The cricothyroid artery arises from the superior thyroid artery itself, the first branch given off from the external carotid artery, and crosses the upper cricothyroid membrane, which extends from the cricoid cartilage to the thyroid cartilage. The superior thyroid artery is found along the lateral edge of the cricothyroid membrane. When planning a cricothyrotomy, the anatomy of the cricothyroid artery and the thyroid artery should be considered but rarely should affect the practice. It is best to stay in the midline, midway between the cricoid and thyroid cartilages (Morgan et al., 2006).

The vagus nerve (the tenth cranial nerve) provides sensation to the airway below the epiglottis. The superior laryngeal branch of the vagus divides into an external (motor) nerve and an internal (sensory) laryngeal nerve that provide sensory supply to the larynx between the epiglottis and the vocal cords. Another branch of the vagus, the recurrent laryngeal nerve, innervates the larynx below the vocal cords and the trachea (Morgan et al., 2006).

The muscles of the larynx are innervated by the recurrent laryngeal nerve with the exception of the cricothyroid muscle, which is innervated by the external (motor) laryngeal nerve, a branch of the superior laryngeal nerve. The posterior cricoarytenoid muscles abduct the vocal cords, whereas the lateral cricoarytenoid muscles are the principal adductors (Morgan et al., 2006).

\section{A-Airway injuries:-}

laryngoscopy and intubation can lead to a range of complications from sore throat to tracheal stenosis. Injury to airway structures is a constant concern to practicing anesthesiologists. The daily insertion of endotracheal tubes, laryngeal mask airways, oral/nasal airways, gastric tubes, transesophageal echocardiogram (TEE) probes, and emergency airways all involve the risk of airway structure damage. Common morbidities such as sore throat and dysphagia are usually self-limiting but may also be nonspecific symptoms of more ominous complications (Schneider T et al., 2007).

Excessive flexion while placing the head in the sniffing position can stresses the retaining ligaments of the ventrally placed odontoid process (C1-C2 complex), most important in atlantoaxial instability. On the other hand, when the anesthetist place one hand on the chin, with the other on the occipitoparietal area of the cranium and pushing the head into extreme extension. (Whylie et al.,1995).

The most common permanent airway injury is dental trauma. Other types of airway trauma are rare. Although there are scattered case reports in the literature, the most comprehensive analysis is the ASA Closed Claims project. This report describes 266 claims, which were grouped by the site of injury. In general, the least serious were tempromandibular joint (TMJ) injuries, which were all associated with otherwise uncomplicated intubations and occurred mostly in females younger than 60 years. Approximately $25 \%$ of these patients had previous TMJ disease. Laryngeal injuries primarily included vocal cord paralysis, granuloma, and arytenoid dislocation. Most tracheal injuries were associated with emergency surgical tracheotomy, but a few were related to endotracheal intubation. Some injuries occurred during seemingly easy, routine intubations. Proposed mechanisms include excessive tube movement in the trachea, pressure necrosis, and inadequate relaxation (Schneider T et al., 2007).

Instrumentation with a metal laryngoscope blade and insertion of a stiff tracheal tube (TT) often traumatize delicate airway tissues. Although tooth damage is the most common cause of malpractice claims against anesthesiologists Postintubation croup caused by glottic, laryngeal, or tracheal edema is particularly serious in children. The efficacy of corticosteroids (e.g., dexamethasone $0.2 \mathrm{mg} / \mathrm{kg}$, up to a maximum of $12 \mathrm{mg}$ ) in preventing postextubation airway edema remains controversial; however, they have been demonstrated to be efficacious in children with croup from other causes. Vocal cord paralysis from cuff compression or other trauma to the recurrent laryngeal nerve results in hoarseness and increases the risk of aspiration. Some of these complications may be decreased by using a TT shaped to conform to the anatomy of the airway (e.g., Lindholm Anatomical Tracheal Tube). The incidence of postoperative hoarseness appears to increase with obesity, difficult intubations, and anesthetics of long duration. Applying a water-soluble lubricant or an anesthetic-containing gel to the tip or cuff of the TT does not decrease the incidence of postoperative sore throat or hoarseness. Smaller tubes (size 6.5 in women and size 7.0 in men) are associated with fewer complaints of postoperative sore throat. Repeated attempts at laryngoscopy during a difficult intubation may lead to periglottic edema and the inability to ventilate with a face mask, thus turning a bad situation into a life-threatening one (Morgan et al., 2006). 


\section{1 - Dental injuries}

The most common permanent airway injury during general anesthesia is dental trauma. In most cases, laryngoscopy and endotracheal intubation are the cause, and the upper incisors were the most frequently injured. Dental trauma may occur less commonly from oral airways. Major risk factors for dental trauma included tracheal intubation, preexisting poor dentition, and patient characteristics associated with difficult airway management (including limited neck motion, previous head and neck surgery, craniofacial abnormalities, and a history of difficult intubation) (Lockhart PB et al., 1996).

\section{2- Lips and oropharyngeal mucous membrane injury}

Lip injuries are frequently encountered during routine practice of GA, conceivably attributable to preexistent desiccation of the lips (in the absence of any direct contact with the instruments and medical conditions that cause xerosis of skin and mucosa). Such injuries are potentially preventable possibly by the local use of emollients for few days prior to the GA. Nonetheless, only modest concerns have been raised in regard to the factors involved in the injuries to the lips and their prevention. Lip injuries have significant physical, aesthetic and emotional consequences following the recovery of the patients from GA (Arnold HL et al., 1990).

Table I-1:-The Effects of Laryngeal Nerve Injury on the Voice.

\begin{tabular}{|l|l|}
\hline Nerve & Effect of Nerve Injury \\
\hline Superior laryngeal nerve & \\
\hline $\begin{array}{l}\text { Unilateral } \\
\text { Bilateral }\end{array}$ & $\begin{array}{l}\text { Minimal effects } \\
\text { Hoarseness, tiring of voice }\end{array}$ \\
\hline Recurrent laryngeal nerve & \\
\hline $\begin{array}{l}\text { Unilateral } \\
\text { Bilateral }\end{array}$ & Hoarseness \\
Acute & Stridor, respiratory distress \\
Chronic & Aphonia \\
\hline Vagus nerve & \\
\hline Unilateral & Hoarseness \\
Bilateral & Aphonia \\
\hline
\end{tabular}

\section{3- Vocal cords}

Phonation involves complex simultaneous actions by several laryngeal muscles. Damage to the motor nerves innervating the larynx leads to a spectrum of speech disorders (Table I-1). Unilateral denervation of a cricothyroid muscle causes very subtle clinical findings. Bilateral palsy of the superior laryngeal nerve may result in hoarseness or easy tiring of the voice, but airway control is not jeopardized (Morgan et al., 2006).

Unilateral paralysis of a recurrent laryngeal nerve results in paralysis of the ipsilateral vocal cord, causing deterioration in voice quality. Assuming intact superior laryngeal nerves, acute bilateral recurrent laryngeal nerve palsy can result in stridor and respiratory distress because of the remaining unopposed tension of the cricothyroid muscles. Airway problems are less frequent in chronic bilateral recurrent laryngeal nerve loss because of the development of various compensatory mechanisms (e.g., atrophy of the laryngeal musculature). Bilateral injury to the vagus nerve affects both the superior and the recurrent laryngeal nerves. Thus, bilateral vagal denervation produces flaccid, midpositioned vocal cords similar to those seen after administration of succinylcholine. Although phonation is severely impaired in these patients, airway control is rarely a problem (Morgan et al., 2006).

\section{4 - Tracheal injuries:}

Most of these are due to prolonged external pressure on sensitive airway structures. When these pressures exceed the capillary-arteriolar blood pressure (approximately $30 \mathrm{~mm} \mathrm{Hg}$ ), tissue ischemia can lead to a sequence of inflammation, ulceration, granulation, and stenosis. Inflation of a TT cuff to the minimum pressure that creates a seal during routine positive-pressure ventilation (usually at least $20 \mathrm{~mm} \mathrm{Hg}$ ) reduces tracheal blood flow by $75 \%$ at the cuff site. Further cuff inflation or induced hypotension can totally eliminate mucosal blood flow (Morgan et al., 2006). 
Tracheal laceration is a rare but potentially devastating complication of endotracheal intubation. Traditional management of intubation-related tracheal laceration is operative. Nonoperative management of a woman noted to have a tracheal laceration during intubation is described. Criteria by which nonoperative treatment can be considered are outlined (Howard M. Ross et al, 1996).

Iatrogenic tracheal rupture is a dangerous complication with potentially high postoperative mortality, mostly influenced by the underlying disease. Early surgical repair must be the preferred treatment (Hofmann H.S. et al, 2002).

\section{5 - Oesophageal injuries:}

Esophageal perforation is a serious life-threatening injury that may occur during inadvertent esophageal intubation or several attempts of difficult endotracheal intubation. Early diagnosis of this complication is vital to successful treatment and requires a high index of suspicion because the symptoms are often nonspecific and may be delayed (Younes Z and Johnson D, 1999).

Esophageal perforations contributed to death in little number of patients, who most often presented with delayedonset subcutaneous emphysema or pneumothorax.Pharyngoesophageal perforation was associated with difficult intubation, age over 60 years, and female gender. As in tracheal perforation, obvious signs were often delayed in onset. Instead, initial sore throat, cervical pain, and cough often progress to fever, dysphagia, and dyspnea as mediastinitis, abscess, or pneumonia develop. Mortality rates of 25-50\% after esophageal perforation have been reported, with the lower percentage attributable to rapid detection and treatment (White RK and Morris DM, 1992)

Minimizing the risk of airway injury begins with the preoperative assessment. A thorough airway examination will help determine the risk for difficulty. Documentation of current dentition (including dental work) should be included. Many practitioners believe preoperative consent should include a discussion of the risk of dental, oral, vocal cord, and esophageal trauma in every patient who could potentially need any airway manipulation. If a difficult airway is suspected, a more detailed discussion of risks (e.g., emergency tracheostomy) is appropriate. In such cases, emergency airway supplies and experienced help should be immediately available, and the ASA algorithm for difficult airway management should be utilized. Follow-up should occur to assess for latent signs of perforation if there is suspicion of airway trauma. If intubation cannot be accomplished by conventional means, the patient or guardian should be informed in case of future airway intervention (T. Schneider et al., 2007).

\section{B-Positioning related injuries}

Changes of body position have physiological consequences that can be exaggerated in disease states . General and regional anesthesia may limit the cardiovascular response to such a change. Even positions that are safe for short periods may eventually lead to complications in persons who are not able to move in response to pain. For example, the alcoholic patient who passes out on a hard floor may waken with a brachial plexus injury. Similarly, regional and general anesthesia abolish protective reflexes and predispose patients to injury (Atlee AL, 1999).

Many complications, including air embolism caused by the physiological changes described above, nerve damage as a result of ischemic injury, and the need for finger amputation following a crush injury, have been associated with improper patient positioning (Table I-2). These complications are best prevented by evaluating the patient's postural limitations during the preanesthetic visit; padding pressure points, susceptible nerves, and any area of the body that will possibly be in contact with the operating table or its attachments; avoiding flexion or extension of a joint to its limit; having an awake patient assume the position to ensure comfort; and understanding the potential complications of each position. it is possible at any stage of the positioning process for a patient to be dropped by attendants involved in either positioning or transfer duties. In positioning the patient, neck injury can be produced in a variety of ways, probably the best known one is whiplash injury which occurs when support for the head is suddenly

Table 1-2. Complications Associated with Patient Positioning. (Morgan et al., 2006).

\begin{tabular}{|l|l|l|}
\hline Complication & Position & Prevention \\
\hline Air embolism & $\begin{array}{l}\text { Sitting, prone, reverse } \\
\text { Trendelenburg }\end{array}$ & $\begin{array}{l}\text { Maintain venous pressure above 0 at the } \\
\text { wound. }\end{array}$ \\
\hline Alonecia & Sunine. lithotomv. & Normotension. padding. and occasional \\
\hline
\end{tabular}




\begin{tabular}{|l|l|l|}
\hline & Trendelenburg & head turning. \\
\hline Backache & Any & $\begin{array}{l}\text { Lumbar support, padding, and slight hip } \\
\text { flexion. }\end{array}$ \\
\hline $\begin{array}{l}\text { Compartment } \\
\text { syndrome }\end{array}$ & Especially lithotomy & $\begin{array}{l}\text { Maintain perfusion pressure and avoid } \\
\text { external compression. }\end{array}$ \\
\hline Corneal abrasion & Especially prone & Taping and/or lubricating eye. \\
\hline Digit amputation & Any & $\begin{array}{l}\text { Check for protruding digits before } \\
\text { changing table configuration. }\end{array}$ \\
\hline Nerve palsies & & $\begin{array}{l}\text { Avoid stretching or direct compression at } \\
\text { neck or axilla. }\end{array}$ \\
\hline Brachial plexus & Any lateral & Pad lateral aspect of upper fibula. \\
\hline Common peroneal & Lithotomy, \\
decubitus & Any & Avoid compression of lateral humerus. \\
\hline Radial & Any & Padding at elbow, forearm supination. \\
\hline Ulnar & Prone, sitting & Avoid pressure on globe. \\
\hline Retinal ischemia & Any & Padding over bony prominences. \\
\hline Skin necrosis &
\end{tabular}

withdrawn. Periods of patient transport pose a particular threat if monitoring is interrupted for any reason. Similarly, monitors often must be disconnected during patient repositioning (Morgan et al., 2006).

Peripheral nerve injuries appear to be more closely related to positioning or surgical procedure. They may involve the ulnar nerve, peroneal nerve, the brachial plexus, or the femoral and sciatic nerves. External pressure on a nerve could compromise its perfusion, disrupt its cellular integrity, and eventually result in edema, ischemia, and necrosis. Pressure injuries are particularly likely when nerves pass through closed compartments formed by dense osseofascial membranes or take a superficial course (e.g., the perineal nerve around the fibula). Lower extremity neuropathies, particularly those involving the peroneal nerve, have been associated with improper, extreme (high), and prolonged (greater than $2 \mathrm{~h}$ ) maintenance of the lithotomy position. Patient risk factors for this complication include hypotension; thin body habitus; old age; and history of vascular disease, diabetes, or smoking. Ulnar nerve injuries are associated with cardiac surgery because rib retraction may promote stretch on the brachial plexus. Similarly, the long thoracic nerve may be severed during pneumonectomy or axillary lymph node dissection, resulting in paralysis of the serratus anterior muscle and winging of the scapula. Some brachial plexus injuries following lateral decubitus positioning may be related to improper positioning of the axillary roll. This roll should be caudad to the axilla to prevent direct pressure on the brachial plexus, and large enough to relieve any pressure from the mattress on the lower shoulder (Morgan et al., 2006).

The data suggest that some peripheral nerve injury is not preventable. Patient, procedure, and position factors contribute a significant amount of risk. Although the risk of peripheral neuropathy should be discussed during informed consent, particularly in patients to be placed in a nonsupine position, other practices may be helpful. When reasonable, patients can be positioned before induction of anesthesia to check for discomfort. Final positioning should be evaluated carefully prior to draping. In most circumstances the head and neck should be kept in a neutral position to minimize neural or vascular compromise. Shoulder braces to support a Trendelenburg position should be avoided if possible, and shoulder abduction and lateral rotation should be minimized to reduce the chance of brachial plexus injury. The upper extremities should not be extended greater than $90^{\circ}$ at any joint and should be supinated to protect the ulnar tunnel. Prolonged pronation of the forearm can compress the ulnar nerve in the cubital tunnel. Lower extremities should not have any obvious pressure points. Although injuries can occur despite the presence of padding, additional padding may be helpful in vulnerable areas. Documentation should include information on positioning, including the presence of padding. Finally, patients who complain of sensory or motor dysfunction in the postoperative period should be reassured that this is frequently a temporary condition. Motor and 
sensory function should be documented and the patient should be referred for neurological evaluation and physiological testing, such as nerve conduction and electromyographic studies (Morgan et al., 2006).

Anatomically, many of the body's muscles exert their force on tendons that glide around bony tubercles or through tendonous retinacula. This enables the muscles to exert their actions around joints whether the joints are extended or flexed. Some nerves follow similar anatomic courses (Coppieters MW et al., 2002).

Five in vivo mechanisms for perioperative peripheral neuropathies are stretch, compression, generalized ischemia, metabolic derangement, and surgical section (Dylewsky W \& McAlpine FS, 1997). As little as $10 \%$ to $15 \%$ elongation of a peripheral nerve is sufficient to cause changes in vital physiologic processes in a peripheral nerve (Coppieters MW et al., 2002).

Metabolic diseases play a role in perioperative neuropathies such as diabetes, and nutritional problems such as pernicious anemia and alcoholic neuritis are common in surgical patients. Arteriosclerosis, drug, or heavy metal exposure and infectious diseases such as polio also can cause neuropathies. Perioperative cigarette smoking is associated with an increased incidence of postoperative neuropathies, presumably because of the vasoconstrictive effects of nicotine (Warner MA et al., 1994).

\section{Upper Extremity Nerve Injury During Anesthesia}

\section{(1) Brachial Plexus:}

Brachial plexus injuries occur primarily in cardiothoracic procedures requiring median sternotomy.

Mechanisms for brachial plexus injury during median sternotomy include stretch or compression of the plexus during sternal separation, direct trauma from fractured 1st ribs, stretching related to internal mammary dissection, and trauma or haematoma related to internal jugular vein cannulation. Brachial plexus injuries constitute $20 \%$ of claims for nerve injury in the ASA Closed Claims Database (Cheney FW et al., 1999).

A prospective study of patients undergoing open heart surgery found the incidence of these injuries to be as high as $4.9 \%$. Lower parts of the plexus are most often involved, producing painless motor deficits that usually resolve over 6 to 8 weeks (Dylewsky W \& McAlpine FS, 1997).

Shoulder braces placed close to the neck of patients undergoing noncardiothoracic procedures in a steep head-down (Trendelenburg) position may put pressure directly on the upper roots and trunks of the brachial plexus. Braces placed more laterally may put excessive traction on the brachial plexus by displacing the shoulder inferiorly. Coppieters and coworkers studied the effect of the brachial plexus tension test (BPTT), a clinical equivalent of straight leg raising in the upper extremity, in asymptomatic male volunteers. Their results led them to strongly discourage the use of shoulder braces in positioning patients for surgery. Nonsliding mattresses were recommended when shoulder braces were thought absolutely required for a steep head-down tilt (Coppieters MW et al., 2002).

The prone position has also been implicated in brachial plexus injuries. Although theoretical advantages may support tucking the arms at the side in prone patients or turning the head to the side of an arm abducted up, no data support this idea. Brachial plexus injuries in prone patients are rare (Coppieters MW et al., 2002).

\section{(2) Ulnar Neuropathy:}

The most common peripheral nerve injury is ulnar neuropathy.Ulnar neuropathy was formerly attributed to intraoperative compression or stretching because of the nerve's vulnerable position at the elbow where it passes around the medial epicondyle of the humerus and under the retinaculum of the cubital tunnel (Contreras MG et al., 1998).

The anatomic boundaries of the cubital tunnel are the floor (i.e., medial ligament of the elbow) and the roof (i.e., arcuate ligament, which extends from the medial epicondyle of the humerus to the medial aspect of the olecranon process of the ulna). The ulnar nerve then passes posteromedially to the tubercle of the coronoid process of the ulna; the arterial supply of the nerve in this area, the posterior recurrent ulnar artery, is very superficial. Anatomically, the tubercle of the coronoid process is 1.5 times larger in men and boys, who also are more likely to have a thicker cubital tunnel retinaculum. Women and girls have a much thicker layer of subcutaneous fat between the skin and the ulnar nerve at the cubital tunnel and the medial aspect of the coronoid process (Contreras MG et al., 1998). 
An exhaustive retrospective study of the Mayo Clinic database for perioperative ulnar neuropathies occurring over a 35-year period (1957-1991) was reported in 1994. Ulnar neuropathies lasting longer than 3 months were identified in 414 of 1,129,692 consecutive patients ( 1 per 2729 patients) undergoing procedures requiring anesthesia or monitored anesthesia care. Seventy percent of the patients developing ulnar neuropathy were male. Almost half of the deficits were sensory only, and $80 \%$ of these patients regained complete sensation in 1 year. Only $35 \%$ of patients with mixed sensory and motor deficits recovered completely in 1 year. Warner and coworkers concluded that perioperative ulnar neuropathies might not be related to patient positioning for several reasons. Symptoms were not observed for more than 24 hours after the procedure in 57\% of patients. Many of the neuropathies occurred in patients who did not receive general anesthesia. Bilateral ulnar neuropathies occurred in $9 \%$. There were strong associations with several patient characteristics: male gender, very obese or very thin body habitus, and prolonged hospitalization (>14 days) (Warner MA et al., 1994).

Two prospective studies further strengthened the suspicion that the injury associated with postoperative ulnar neuropathy does not usually occur intraoperatively. In the first, 1502 surgical patients were carefully assessed for neurologic signs and symptoms before and after surgery. It was found that seven patients ( 1 in 215) developed ulnar neuropathy perioperatively, but that none of the 7 was symptomatic within the first 2 postoperative days. The median onset of symptoms was the fourth postoperative day. Six of seven of the patients were men (Warner MA et al., 1999).

In another prospective study of medical patients requiring no surgical procedures, 2 of 986 patients developed ulnar neuropathy (Warner MA et al., 2000).

It has become accepted that postoperative ulnar nerve palsy can occur without any apparent cause, even when positioning and padding of the patient's arms has been carefully done perioperatively. Postoperative ulnar nerve palsy is not always a preventable complication (Stoelting RK, 1993).

\section{(3) Other Upper Extremity Nerve Injuries:}

The radial nerve may be injured where it wraps laterally around the middle of the humerus or laterally in its groove at the elbow. More muscular patients may be at higher risk because of limitations of extension of the elbow caused by hypertrophied biceps muscles and inflexible tendons. This may put the median or the anterior interosseous nerve on a greater stretch intraoperatively (Contreras MG et al., 2002).

Transient median nerve carpal tunnel symptoms were identified in 27 of 1502 patients followed prospectively by Warner and coworkers[ during the perioperative period. The anterior interosseous nerve contains no sensory fibers, but loss of it produces weakness of the thumb and index finger and a characteristic square pinch rather than an "O" on opposition of the thumb and index finger. Specific sensory and motor deficiencies caused by upper and lower extremity neuropathies are listed in ( Warner MA et al., 1999).

\section{Lower Extremity Neuropathies}

A large, retrospective study found motor neuropathies persisting more than 3 months in 55 patients among those undergoing 198,461 procedures in the lithotomy position, which is an incidence of 1 in 3608 (Warner MA et al., 1994).

Forty-three of the 55 neuropathies involved the common peroneal nerve. Motor function of the affected nerve recovered in $43 \%$ of the patients within 1 year. Three risk factors were strongly associated with increased risk of developing a neuropathy in the lithotomy position: prolonged surgery ( $>3$ hours), very thin body habitus, and recent cigarette smoking (Warner MA et al., 1994).

Warner and coworkers also prospectively studied lower extremity neuropathies in 991 lithotomy patients. Fifteen patients $(1.5 \%)$ developed neuropathies. The obturator, lateral femoral cutaneous, sciatic, and peroneal nerves were involved in five, four, three, and three patients, respectively (Warner MA et al 2000).

Fourteen of 15 patients presented with paresthesias; the symptoms resolved within 6 months in all but 1 patient. Prolonged positioning in the lithotomy position ( $>2$ hours) and the extremes of body weight were found to contribute to an increased risk for lower extremity neuropathies after surgery in the lithotomy position in this prospective study. Transient neurologic symptoms, also referred to as transient radicular irritation, can manifest as 
severe pain radiating down both legs after spinal anesthesia with lidocaine. The lithotomy position is associated with more frequent occurrences of this problem ( Pollock JE et al., 1996).

The common peroneal nerve passes around the head of the fibula laterally, where it can be injured by compression by leg holders used to position patients in a lithotomy position. The sciatic nerve can be stretched by hyperflexion of the hip and extension of the knee in patients in a lithotomy position. The femoral nerve can be injured by compression by abdominal wall retractors. The obturator nerve passes through the pelvis and is occasionally injured by surgical retractors placed deep below the pelvic brim. Excessive flexion can also injure the obturator nerve (Warner MA et al 2000).

The lateral femoral cutaneous nerve is sensory only. Excessive flexion of the hip on the abdomen may occasionally cause compression leading to temporary neuropathies (Warner MA et al 2000).

\section{[C] Ophthalmic injuries}

Eye complications are frustratingly common in patients who have undergone procedures in the prone position and are at risk in other positions as well. Eye complications constitute $3 \%$ of all claims in the ASA Closed Claims Database but have a higher frequency of payment than that for claims for other conditions. Corneal abrasions are most common, often involving the "down eye" in patients who were positioned prone with their heads turned to the side. One study put the prevalence at $0.17 \%$ for prone patients and found taping just after induction of anesthesia to be more effective than ophthalmic ointment used intraoperatively (Cucchiara RF \& Black S, 1988).

Although direct trauma can occur to the cornea, swelling of the dependent eye may be the mechanism causing the down eye to open slightly, allowing a slit-shaped area of cornea to dry. The patient with a corneal abrasion complains of eye pain and a foreign body sensation on awakening from anesthesia. Corneal abrasions usually heal quickly; the affected eye can be patched and antibiotic ointment applied to prevent bacterial infection that could lead to a corneal ulcer (Lee LA et al., 2006).

Pressure on the eyes should always be avoided. Small increases in pressure may occlude venous flow in the retina. If the pressure is slightly higher, arterial flow can be impaired and contribute to ischemia of the retina or optic nerve. Retinal artery occlusion usually manifests as a painless, monocular blindness with a pale edematous retina and a cherry-red spot at the fovea (Lee LA et al., 2006).

Ischemic optic neuropathy (ION) has been recognized as a serious complication of hemorrhage in cardiovascular, spinal, and other types of surgery, leading to blindness in one or both eyes postoperatively (Williams EL et al., 1995).

The association between blood loss, hypotension, and blindness was mentioned by Hippocrates. ION is caused by infarction or ischemia of the anterior or posterior parts of the optic nerve. The blood supply to the posterior optic nerve is a watershed zone where delicate connections exist between the small arterial branches of the central retinal artery and outer pial vessels from branches of the ophthalmic artery (Williams EL et al., 1995).

Many patients with ION do not have atherosclerosis and other cardiovascular risk factors; the complication has been reported in patients as young as 13 years (Brown RH et al., 1994).

In an extensive review in patients undergoing cardiopulmonary bypass, ION was diagnosed in 17 of 27,915 patients $(0.06 \%)$ (Nuttall GA et al., 2001). Four cases occurred in 501,342 anesthetics (0.0008\%) not involving cardiopulmonary bypass at the same institution (Warner ME et al., 2001).

Other factors such as stretch and compression of the optic nerve due to orbital swelling in the prone patient may be involved in ION. Significant swelling often occurs in the orbits after prolonged procedures done in the prone position; this swelling could affect circulation in the orbit posterior to the globe or cause stretching of the ophthalmic nerves (Nuttall GA et al., 2001).

Cortical blindness from hemorrhagic or embolic infarctions of the occipital lobe also occurs in the perioperative period, most commonly in patients after cardiac and neurosurgical procedures. Injuries or bleeding in the optic chiasm can lead to blindness after pituitary surgery (Williams EL et al., 1995). 


\section{Ventilator related injuries:-}

Barotrauma and volutrauma was the most frequent and easily recognized complication of mechanical ventilation in anesthesia. However, now evident is that barotraumas and volutrauma represents only two of the mechanisms underlying the broad category of ventilator-induced lung injury. As the term suggests, the lung injury associated with barotrauma is mediated by increased alveolar pressures and volutrauma is Damage caused by over-distension.

(Pinhu L et al, 2003).

Investigations of barotrauma associated with the use of anesthesia ventilators emphasize the need to bring two problems to the attention of users. The first problem concerns an operating characteristic of anesthesia ventilators with rising bellows. The second problem can develop if the airway hose from the breathing circuit to the ventilator is occluded (Lefor T, 1998).

Other manifestations of ventilator associated injuries have been termed atelectotrauma, and biotrauma (cytokine and chemokine mediated) to reflect the major pathophysiologic events behind the injury (Pinhu $\mathbf{L}$ et al, 2003).

\section{(A)High Pressures from Rising Bellows}

Unlike earlier (descending) bellows, the rising bellows must be filled before patient ventilation can be started. If the ventilator is on and in its inspiratory phase when the flush valve is used to fill the bellows(either at the start of a case or, for example, following disconnection to suction the patient), the patient may be exposed to high pressures in the breathing circuit until the ventilator cycles into exhalation. The problem can be avoided by filling the bellows only when the ventilator is turned off (Lefor T, 1998).

When the ventilator is in its inspiratory phase, driving gas (at a pressure of up to $65-75 \mathrm{~cm} \mathrm{H}_{2} \mathrm{O}$ ) enters the bellows assembly to press the bellows down to deliver a breath. If the flush valve is activated to fill an empty bellows during this phase, the driving gas will keep the bellows from rising until the pressure in the breathing circuit exceeds the driving pressure. This exposes the patient to the maximum available driving system pressure, which some patients will not be able to tolerate (Lefor T, 1998).

If the bellows is filled before the ventilator is turned on or when it is in standby mode, no driving gas pressure is applied to the bellows assembly, so the bellows can be filled with a minimal rise in breathing circuit pressure. This is the only acceptable way to avoid delivering the ventilator's maximum pressure (Lefor T, 1998).

Most anesthesiologists recognize the danger of high breathing circuit pressures from using a flush valve in conjunction with an anesthesia ventilator. However, because the bellows does not rise when users attempt to fill it in the described situation, users may not recognize the high pressures being generated. A user-adjustable high-pressure alarm will give some measure of safety. However, if the alarm is set too high or the user does not respond to it quickly, the protection is lost (Lefor T, 1998).

\section{(B) Breathing Circuit Occlusion:}

If the airway hose from the breathing circuit to the ventilator is occluded, this eliminates all pressure-limiting features from the patient portion of the breathing circuit. (When using an anesthesia ventilator, all pressure-limiting and alarm functions are typically in the ventilator.) Fresh gas flow from the anesthesia unit to the patient circuit can cause a rapid pressure increase because there is no place for the gas to escape (Lefor T, 1998).

A typical fresh anesthesia gas flow $(4-6 \mathrm{~L} / \mathrm{min})$ can raise the breathing circuit pressure to a hazardous level $(90 \mathrm{~cm}$ $\mathrm{H}_{2} \mathrm{O}$ or more) in 10-20 sec. The anesthesiologist's attention may easily be diverted to other functions (e.g., obtaining a manual blood pressure measurement) for this period of time, exposing the patient to these pressures before the problem is recognized and corrective action is taken. If the flush valve is activated when this airway hose is occluded, pressures will rise so high and so quickly that barotrauma can occur before the operator can respond(Lefor T, 1998).

Occlusion of this section of airway hose is highly unlikely in normal use. However, several types of occlusions that occurred in ventilator circuits were reported:

1. A ventilator hose fitting was occluded because of a molding error that resulted in mold flash across the lumen.

2. A misplaced one-way valve disk occluded the ventilator connection to the breathing circuit. 
3. Misconnection of a vacuum line directly to an anesthesia ventilator scavenging port disabled the overflow (and pressure-relief) valve.

4. A breathing circuit filter (such as those often used in anesthesia ventilator hose circuits) became occluded when water entered it and wet the entire filter medium.

5. The occlusion problems described are not unique to any particular manufacturer or anesthesia ventilator model. Occlusion can occur with all types of anesthesia ventilators, not just the rising-bellows type (Lefor T, 1998).

In some cases, occlusion may occur unexpectedly and suddenly (e.g., item 2 above), and there may be no way of preventing the incident. Constant vigilance and readiness to respond may minimize the hazard to the patient. More often, occlusion is likely to be present when the system is set up (e.g., item 1) or to develop over time (e.g., a filter, such as the one described in item 4, that gradually occludes with use). Appropriate pre-use testing must be conducted to identify occlusions present at the time of setup so that they can be corrected before a patient is connected. Gradual occlusions will result in increasing breathing circuit pressure, which must be investigated and corrected (Lefor T, 1998).

\section{Recommendations:-}

1. Always fill the bellows of an anesthesia ventilator before turning the unit on (at the beginning of a case or after an interruption of the case). Reactivate alarms and reset alarm limits as appropriate; some units will revert to default limits when turned on.

2. Confirm proper ventilator operation in the pre-use testing of Anesthesia Units.

3. Make sure that all users are familiar with the hazards of activating the flush valve when an anesthesia ventilator is in use and the importance of maintaining constant vigilance and readiness (Lefor T, 1998).

\section{Cannulation related injuries}

[a] injury related to peripheral venous cannulation:

\section{Hematoma}

Is a collection of blood. Can result from failure to puncture the vein when the cannula is inserted or when the cannula is removed. Selection of an appropriate vein and gently applying pressure slightly above the insertion point as you remove the cannula may prevent this $(\mathbf{R o b} \mathbf{T}, \mathbf{2 0 0 4})$.

\section{Extravasation:}

Extravasation is an unintentional injection or leakage of fluid in the perivascular or subcutaneous space. Extravasation may or may not be associated with tissue injury. Extravasation injury results from a combination of factors, including solution cytotoxicity, osmolality, vasoconstrictor properties, infusion pressure, regional anatomical peculiarities, and other patient factors (Gault DT, 1993). There are surprisingly few data regarding the incidence, consequences, and management of extravasation in the anesthesia and intensive care literature. Also, information regarding the potential of any given drug to cause extravasation injury is not widely available (Boyle DM and Engelking C, 1995).

In the perioperative period, extravasation of hyperosmolar solutions, vasoconstrictive substances, or concentrated electrolyte solutions may cause significant tissue necrosis. Tragically, the extravasation injury can sometimes be worse than the condition that originally brought the patient to the hospital. Even amputation of an extremity, although rare, has been reported as a consequence of extravasation injury (Gault DT, 1993).

Tissue damage after extravasation may be slight and may involve a local and limited inflammatory response or may be large and involve necrosis of the skin and underlying soft tissues (Hastings-Tolsma M and Yucha CB, 1994).

The degree of damage depends on the localization of the extravasation, the physicochemical characteristics of the agent administered, and the duration of soft tissue exposure to the agent, as well as on the patient's general health. Pain, swelling, or local hyperthermia are not reliable predictors of the degree of tissue damage (Boyle DM and Engelking C, 1995).

Furthermore, agents that cause pain even during intravascular injection may not necessarily cause tissue injury upon extravasation. As an example, small amounts of propofol may cause substantial pain upon intravascular injection, but even large amounts of propofol cause no damage upon extravasation (Findlay JY, 1994). In contrast, extravasation of substances that subsequently lead to major tissue injury may cause only minor discomfort in the 
early stages of extravasation. The patient in Case 1 mentioned only "some tension" after extravasation of potassium chloride. Paresthesia, local induration of the skin, and ulcers are late signs of extravasation that may take days to develop (Kassner E, 2000). However, an induration lasting longer than 24 hours is a reliable sign for the formation of necrosis. These peculiarities of extravasation injury show that the severity of extravasation injuries is easily misjudged and that inadequate early treatment may result in severe morbidity (Casanova D et al., 2001).

\section{Embolism:}

Embolism can be caused by air, a thrombus, or fragment of a catheter breaking off and entering the venous system. Such things can go on to lodge in an artery, blocking circulation to the corresponding area. To avoid air embolus, make sure that there is no air in the system. To avoid a thromboembolism use a smaller cannula. Avoid the catheter breaking by never reinserting the needle (Rob T, 2004).

\section{Phlebitis:}

Phlebitis is an inflammation of the vein resulting from mechanical or chemical irritation or from an infection. Phlebitis can be avoided by carefully choosing the site for cannulation and by checking the type of infusate you use

(Rob T, 2004).

\section{[B] INJURY RELATED TO CENTRAL VENOUS CANNULATION:} (1) Inadvertent carotid arterial puncture:

This complication is reported to have an incidence of $2 \%-8 \%$ and usually results in localised haematoma formation. The haematoma may enlarge rapidly if the patient is coagulopathic, or if a large puncture wound is produced by the introduction of the sheath itself into the carotid artery. Airway obstruction, pseudoaneurysm, arterio-venous fistula formation and retrograde aortic dissection have all been reported as a consequence of carotid puncture (Kua JS and Tan IK, 1997). In the presence of occlusive (atheromatous) carotid disease, inadvertent puncture may carry the risk of precipitating a cerebrovascular accident (Applebaum RM et al, 1997).

If carotid puncture occurs immediately before surgery, the question of whether or not to proceed is a difficult one. The serious consequences of carotid puncture occurs with using a large bore cannula, if the carotid artery was punctured by a small bore needle or cannula (18-gauge or smaller), then serious damage was unlikely to result. However, if a dilator or PAC was inserted into a coagulopathic patient then the risks of complication were significant and in such cases, the catheter should be removed during exploratory surgery of the neck (Golden LR, 1995).

On the basis of present evidence, the recommended management plan for inadvertent carotid cannulation (with a large bore cannula) should it occur: Initially, leave the cannula or sheath in the vessel and consider the options; If the patient is judged to be at low risk of complication, remove the cannula and apply firm pressure for ten minutes. Monitor the neurological, haemodynamic and airway state during this time and be prepared for emergency intubation. Contemplate proceeding to surgery according to the considerations outlined above (Eckhardt et al, 1996).

If the patient is judged to be at high risk of complication, consider intubating the patient (in order to prevent airway compression) before removing the sheath. Removal of the sheath can then be performed, or, on the recommendation of some authors, can be carried out at an exploratory operation (Eckhardt et al, 1996).

If the sheath is removed, apply carotid pressure for ten minutes, being mindful that this may not be successful due to a coagulopathic state, extensive damage to the vessel or bleeding from a site distal to the puncture site. Remember that pressure on the carotid artery can in itself precipitate a neurological event. This is a powerful argument for recommending that pressure only be applied in a conscious, cooperative patient. Monitor the general state of the patient. Bleeding can occur proximal to the puncture site producing a rapid deterioration in the susceptible patient (Oropello JM et al,. 1996).

Consult a vascular surgeon as soon as is practical to determine if surgical exploration or repair is warranted. Indications for exploration may include an enlarging haematoma, airway compression, pre-existing carotid artery disease, a neurological event or cardiovascular instability. Some authors advise exploration in all patients who have had puncture with 17-gauge or larger needle. Caution is strongly recommended in the patient group more prone to 
complications, damage to other vessels beyond the puncture site has been reported. Hence vigilance is still advised even if exploration of the puncture site has been undertaken (Oropello JM et al,. 1996).

Seriously consider delaying surgery for twenty four hours to allow stabilisation and observation (Golden LR, 1995).

If repeat cannulation is required, avoid venepuncture at the same site. Puncture of the carotid artery may not always be related to poor technique but to anatomical variation and thus repeat cannulation may produce the same result. Neither is it advisable to attempt insertion via the opposite internal jugular vein. - Right handed operators find left internal jugular venepuncture technically difficult and if the internal carotid artery is damaged on this side as well, the patient's cerebral circulation may be significantly compromised. For this reason it is recommended to use the subclavian vein on the contralateral side unless contraindicated (Golden LR, 1995).

\section{(2)Pneumothorax:}

Pneumothorax can complicate internal jugular venepuncture, but is usually reported more commonly with the subclavian approach. However, in one recently reported large series, the incidence of this complication occurred in $2.6 \%$ of IJV cannulations and only $1.3 \%$ of subclavian attempts (Miller JA, 1999).

\section{(3) Venous air embolism:}

Venous air embolism is a rare complication of jugular venepuncture and can occur whenever there is a subatmospheric pressure in the venous system at a point where it is open to the atmosphere. These conditions are most likely exist in a patient in whom the central venous pressure is low and / or the fluctuations in intrathoracic pressure are large. Such conditions would include:

- Jugular venepuncture of patients in the semi-upright or sitting position - unless the central venous pressure is extremely high.

- Jugular venepuncture of patients with unrelieved inspiratory airway obstruction (snoring).

During central venous cannulation, air embolism can usually be prevented by appropriate positioning of the patient.

Seldinger needles incorporate a valved side-arm for introduction of the wire. These needles allow the user to maintain a completely closed system during wire introduction (Albin MS ,1993).

Venous air embolism should be suspected when there is a sudden onset of tachycardia associated with pulmonary hypertension and systemic hypotension. A new murmur caused by turbulent flow in the right ventricular outflow tract may also be heard. The diagnosis can be confirmed by echocardiography. The clinical picture is determined by the total volume of air entering the circulation and the rate of entrainment of air. These two factors determine the fate of the bubbles - whether they dissipate as they traverse the heart, or coalesce, leading to an obstruction of the right ventricular outflow tract (Albin MS ,1993).

Slow entrainment of small volumes of air presents with a gradually rising CVP and PAP. The ETCO2 shows a gradual fall as the air passing into the pulmonary circulation dilutes the alveolar CO2. The ECG at this stage may either show no change or abnormalities such as ventricular ectopic beats, tachycardia, bradycardia, ST segment changes, $\mathrm{P}$ wave abnormalities or heart block. Systemic hypotension and the classical 'millwheel' murmur are late developments (Albin MS ,1993).

In contrast, the patient who has entrained a large volume of air will usually present with cardiovascular collapse. The signs include a sudden increase in CVP and PAP accompanied by a sudden fall in ETCO2, saturation and systolic blood pressure. ECG changes may include any of the changes listed above. The volume of air thought to be lethal in the adult has been estimated to be between 300 and $500 \mathrm{ml}$, entering at a rate of $100 \mathrm{ml} / \mathrm{sec}$. This is easily achieved through a $14 \mathrm{G}$ cannula with a pressure gradient of $5 \mathrm{cmH} 2 \mathrm{O}$ (Mennim P et al., 1992).

The potential for paradoxical embolism is higher in this presentation due to the sudden rise in right heart pressures which may then open a probe-patent foramen ovale. Surprisingly, the time of greatest risk of air embolism appears to be on catheter removal (Mennim $P$ et al., 1992).

\section{(4) Nerve Injury:}

The brachial plexus, stellate ganglion, vagus, accessory, hypoglossal and phrenic nerves are all closely associated with the internal jugular vein. Any of these structures may be injured during cannulation attempts. More commonly, 
local anaesthetic injected at the time of cannulation may transiently block these nerves (Burns S and Herbison GJ, 1996).

\section{(5) Jugular vein thrombosis:}

Jugular vein thrombosis can complicate both pulmonary arterial and central venous cannulation by the jugular route and occurs more commonly than subclavian thrombosis. It is much more likely to occur with prolonged use of catheters or when catheter-related sepsis complicates the clinical picture. The incidence has probably been reduced by heparin coating of catheters (Timsit JF et al, 1998).

\section{(6) Cardiac tamponade:-}

Cardiac perforation from a catheter misplaced in the right heart chambers is a rare but life threatening complication of central venous cannulation. Perforation can lead to rabidly progressive cardiac tamponade and sudden cardiovascular collapse and the diagnosis can be over looked in the commotion of cardiopulmonary resuscitation. Immediate pericardiocentesis is necessary to confirm the diagnosis and to relive the tamponade. Emergency thoracotomy may be necessary if there is a large tear in the wall of the heart. Repositioning catheters that extend below the right third anterior intercostal space (which marks the junctions of the superior vena cava with the right atrium) should prevent this life threatening complication (Paul.Marino et al, 2007).

\section{(7) Other miscellaneous complications:}

Inadvertent vertebral arterial puncture and vertebral arterial cannulation have both been reported after attempted internal jugular cannulation (Irita K et al, 1999). Vertebral arterial puncture has resulted in vertebral arterio-venous fistula on several occasions (Majeski JA, 1999).

Transient Horner's syndrome complicating internal jugular catheterisation has recently been reported (Taskapan H et al, 2001).

\section{Injuries related to regional anesthesia Anatomy} The vertebral column

The spine is composed of the vertebral bones and fibrocartilaginous intervertebral disks (Figure II-1). There are 7 cervical, 12 thoracic, and 5 lumbar vertebra (Figure II-2). The sacrum is a fusion of 5 sacral vertebra, and there are small rudimentary coccygeal vertebra. The spine as a whole provides structural support for the body and protection for the spinal cord and nerves, and allows a degree of mobility in several spatial planes. At each vertebral level, paired spinal nerves exit the central nervous system (Figure II-2).(Morgan et al., 2006). 

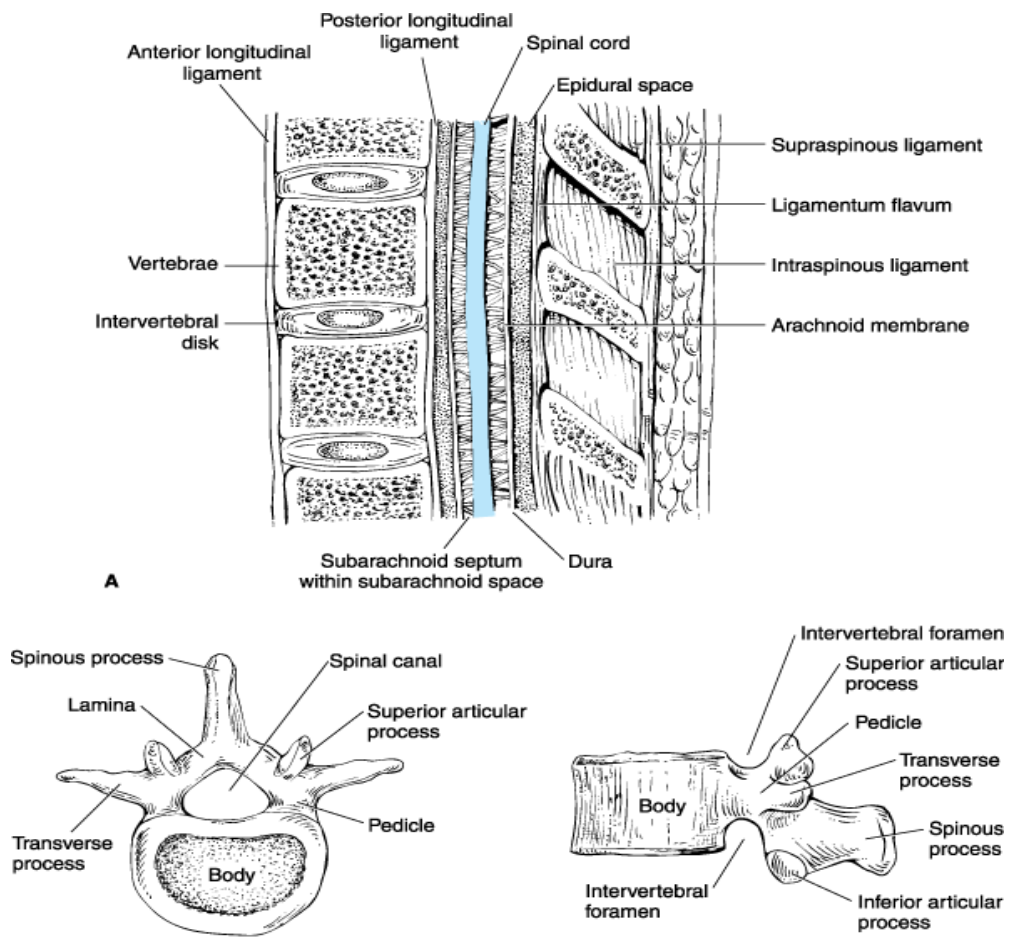

B Superior view
Copyright @2006 by The McGraw-Hill Companies, Inc.
All rights reserved.

c

Lateral view

Sagittal section through lumbar vertebrae (A). Common features of vertebrae (B, C).

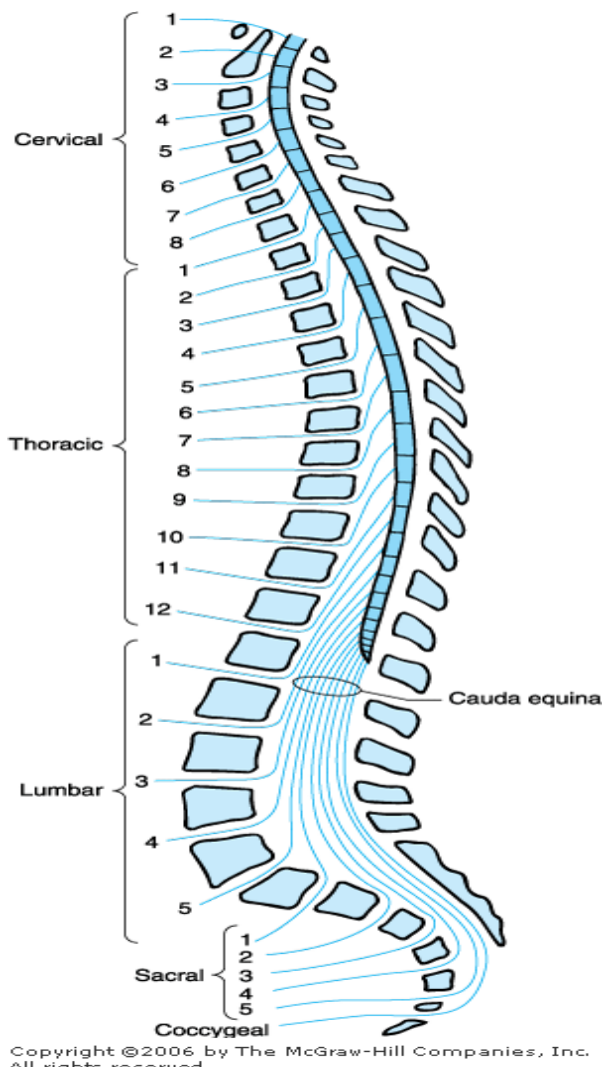

Adapted, with permission, from Waxman SG: Correlative The vertebral column Neuroanatomy, 24th ed 
Vertebra differ in shape and size at the various levels. The first cervical vertebra, the atlas, lacks a body and has unique articulations with the base of the skull and the second vertebra. The second vertebra, also called the axis, consequently has atypical articulating surfaces. All 12 thoracic vertebrae articulate with their corresponding rib. Lumbar vertebrae have a large anterior cylindrical vertebral body. A hollow ring is defined anteriorly by the vertebral body, laterally by the pedicles and transverse processes, and posteriorly by the lamina and spinous processes (Figure II-1B and C). The laminae extend between the transverse processes and the spinous processes and the pedicle extends between the vertebral body and the transverse processes. When stacked vertically, the hollow rings become the spinal canal in which the spinal cord and its coverings sit. The individual vertebral bodies are connected by the intervertebral disks. There are four small synovial joints at each vertebra, two articulating with the vertebra above it and two with the vertebra below. These are the facet joints, which are adjacent to the transverse processes (Figure II-1C). The pedicles are notched superiorly and inferiorly, these notches forming the intervertebral foramina, from which the spinal nerves exit. Sacral vertebrae normally fuse into one large bone, the sacrum, but each one retains discrete anterior and posterior intervertebral foramina. The laminae of S5 and all or part of S4 normally do not fuse, leaving a caudal opening to the spinal canal, the sacral hiatus (Figure II-3). (Morgan et al., 2006).

The spinal column normally forms a double $\mathrm{C}$, being convex anteriorly in the cervical and lumbar regions (Figure II-2). Ligamentous elements provide structural support and together with supporting muscles help maintain the unique shape. Ventrally, the vertebral bodies and intervertebral disks are connected and supported by the anterior and posterior longitudinal ligaments (Figure II-1A). Dorsally, the ligamentum flavum, interspinous ligament, and supraspinous ligament provide additional stability. Using the midline approach, a needle passes through these three dorsal ligaments and through an oval space between the bony lamina and spinous processes of adjacent vertebra (Figure II-4). (Morgan et al., 2006).

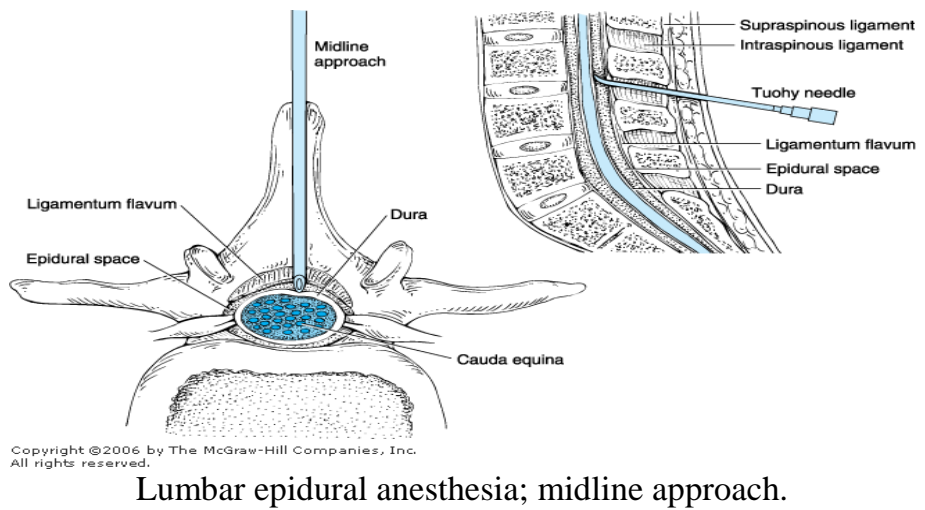

THE SPINAL CORD

The spinal canal contains the spinal cord with its coverings (the meninges), fatty tissue, and a venous plexus (Figure II-5). The meninges are composed of three layers: the pia mater, the arachnoid mater, and the dura mater; all are contiguous with their cranial counterparts (Figure II-6). The pia mater is closely adherent to the spinal cord, whereas the arachnoid mater is usually closely adherent to the thicker and denser dura mater. Cerebrospinal fluid (CSF) is contained between the pia and arachnoid maters in the subarachnoid space. The spinal subdural space is generally a poorly demarcated, potential space that exists between the dura and arachnoid membranes. The epidural space is a better defined potential space within the spinal canal that is bounded by the dura and the ligamentum flavum (Figures II-1 and II-5). (Morgan et al., 2006). 


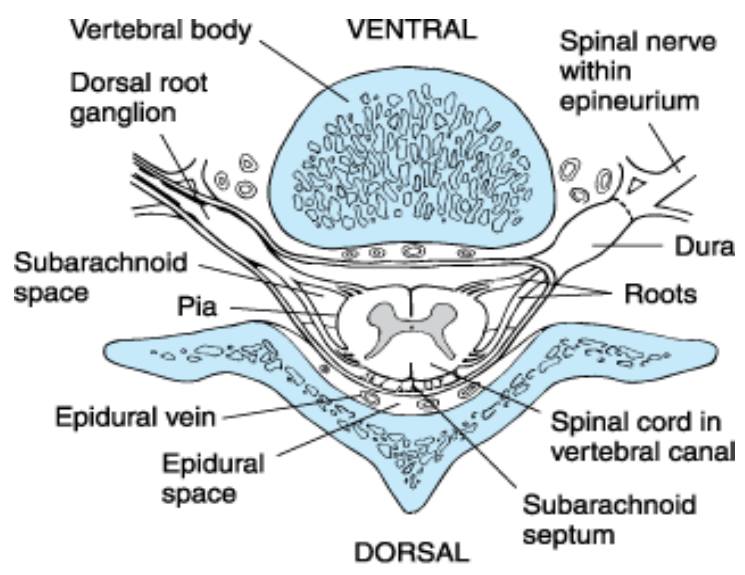

Copyright @2006 by The McGraw-Hill companies, Inc. All rights reserved.

Exit of the spinal nerves.

(Adapted, with permission, from Waxman SG: Correlative Neuroanatomy, 24th ed Figure II-6.

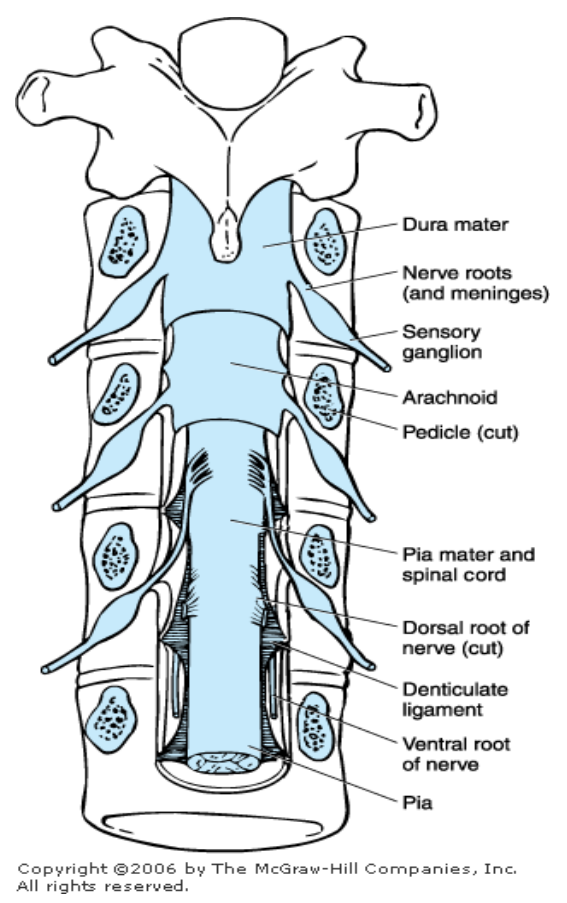

The spinal cord.

The spinal cord normally extends from the foramen magnum to the level of L1 in adults (Figure II-7). In children, the spinal cord ends at L3 and moves up as they grow older. The anterior and posterior nerve roots at each spinal level join one another and exit the intervertebral foramina forming spinal nerves from C1 to S5 (Figure II-2). At the cervical level, the nerves arise above their respective vertebrae, but starting at T1 they exit below their vertebrae. As a result, there are eight cervical nerve roots but only seven cervical vertebrae. The cervical and upper thoracic nerve roots emerge from the spinal cord and exit the vertebral foramina nearly at the same level (Figure II-2). But because the spinal cord normally ends at L1, lower nerve roots course some distance before exiting the intervertebral foramina. These lower spinal nerves form the cauda equina ("horse's tail"; Figure II-2). Therefore, performing a lumbar (subarachnoid) puncture below L1 in an adult (L3 in a child) avoids potential needle trauma to the cord; damage to the cauda equina is unlikely as these nerve roots float in the dural sac below L1 and tend to be pushed away (rather than pierced) by an advancing needle. (Morgan et al., 2006) 


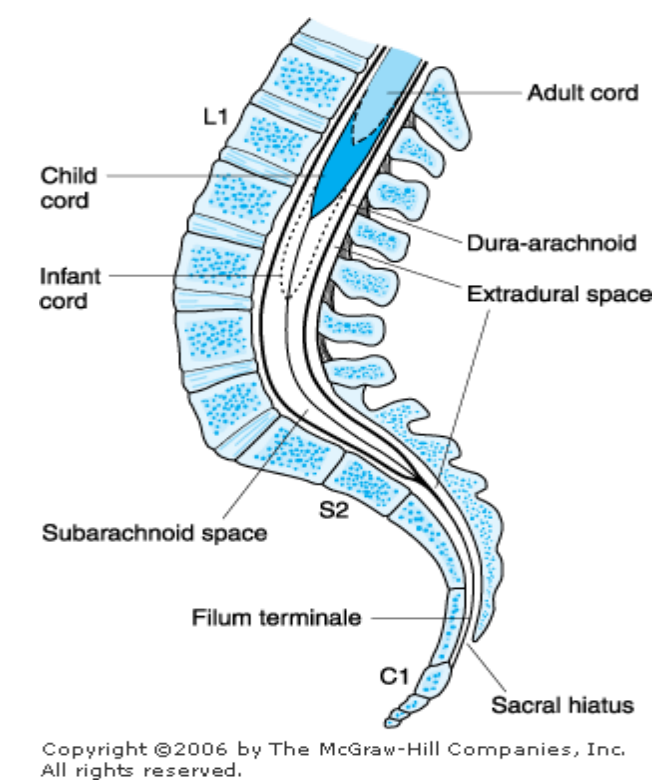

Sagittal view through the lumbar vertebrae and sacrum. Note the end of the spinal cord rises with development from approximately L3 to L1. The dural sac normally ends at S2.

A dural sheath invests most nerve roots for a small distance even after they exit the spinal canal (Figure II-5). Nerve blocks close to the intervertebral foramen therefore carry a risk of subdural or subarachnoid injection. The dural sac and the subarachnoid and subdural spaces usually extend to S2 in adults and often to S3 in children. Because of this fact and the smaller body size, caudal anesthesia carries a greater risk of subarachnoid injection in children than in adults. An extension of the pia mater, the filum terminale, penetrates the dura and attaches the terminal end of the spinal cord (conus medullaris) to the periostium of the coccyx (Figure II-7). (Morgan et al., 2006).

The blood supply to the spinal cord and nerve roots is derived from a single anterior spinal artery and paired posterior spinal arteries (Figure II-8). The anterior spinal artery is formed from the vertebral artery at the base of the skull and courses down along the anterior surface of the cord. The anterior spinal artery supplies the anterior twothirds of the cord, whereas the two posterior spinal arteries supply the posterior one-third. The posterior spinal arteries arise from the posterior inferior cerebellar arteries and course down along the dorsal surface of the cord medial to the dorsal nerve roots. The anterior and posterior spinal arteries receive additional blood flow from the intercostal arteries in the thorax and the lumbar arteries in the abdomen. One of these radicular arteries is typically large, the artery of Adamkiewicz, or arteria radicularis magna, arising from the aorta (Figure II-8A). It is typically unilateral and nearly always arises on the left side, providing the major blood supply to the anterior, lower twothirds of the spinal cord. Injury to this artery can result in the anterior spinal artery syndrome. (Morgan et al., 2006). 


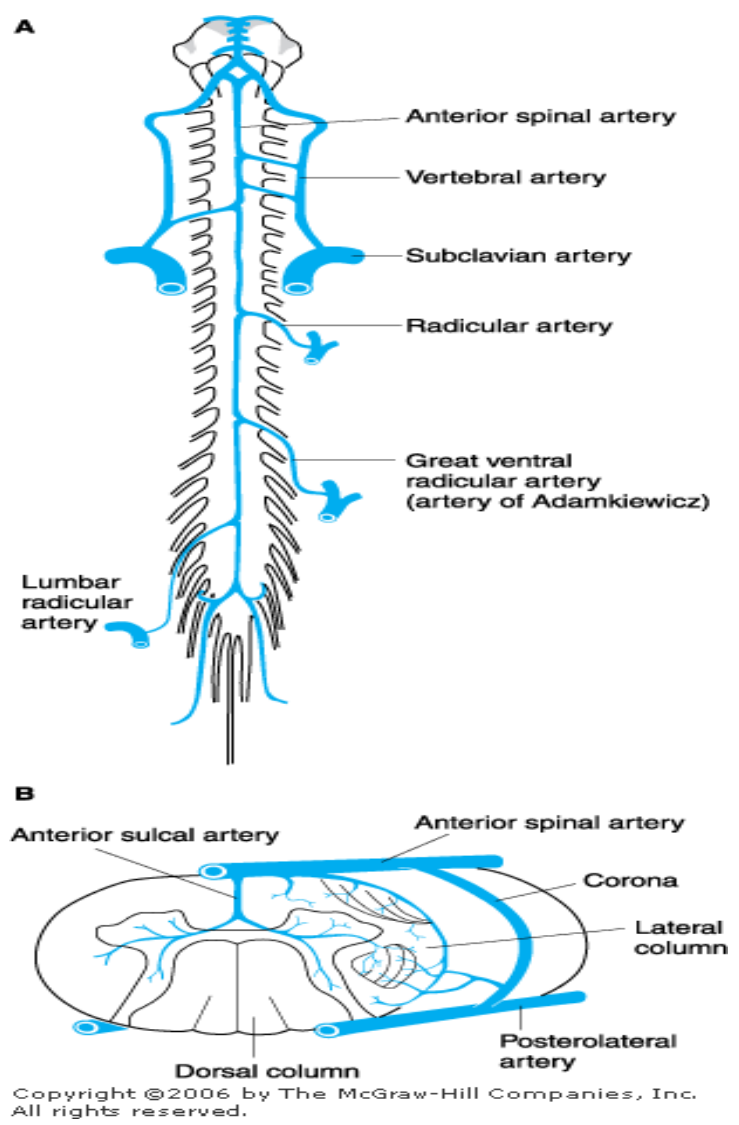

Arterial supply to the spinal cord. Anterior view showing principal sources of blood supply (A). Cross-sectional view through the spinal cord showing paired posterior spinal arteries and a single anterior spinal artery $(\mathbf{B})$.

(Adapted, with permission, from Waxman SG: Correlative Neuroanatomy, 24)

\section{Neurological Injury}

Perioperative nerve injuries may be divided into two categories: those which are unrelated to the regional anesthetic technique (but coincide temporally) and those that are a direct result of the regional anesthetic. Risk factors contributing to neurologic deficit after regional anesthesia include neural ischemia, traumatic injury to the nerves during needle or catheter placement, infection, and choice of local anesthetic solution. However, postoperative neurologic injury due to pressure from improper patient positioning or from tightly applied casts or surgical dressings, as well as surgical trauma are often attributed to the regional anesthetic. Patient factors such as body habitus or a preexisting neurologic dysfunction may also contribute. Prevention of complications, along with early diagnosis and treatment are important in the management of regional anesthetic risks (Auroy Y et al., 1997).

Perhaps no complication is more perplexing or distressing than persistent neurological deficits following an apparently routine neuraxial block in which an epidural hematoma or abscess is ruled out. The nerve roots or spinal cord may be injured. The latter may be avoided if the neuraxial blockade is performed below L1 in adults and L3 in children. Postoperative peripheral neuropathies can be due to direct physical trauma to nerve roots. Although most resolve spontaneously, some are permanent. Some of these deficits have been associated with paresthesia from the needle or catheter or with complaints of pain during injection. Some studies have suggested that multiple attempts during a technically difficult block are also a risk factor. Any sustained paresthesia should alert the clinician to redirect the needle. Injections should be immediately stopped and the needle withdrawn if they are associated with pain. Direct injection into the spinal cord can cause paraplegia. Damage to the conus medullaris may cause isolated sacral dysfunction, including paralysis of the biceps femoris muscles; anesthesia in the posterior thigh, saddle area, or great toes; and loss of bowel or bladder function. Some animal studies suggest catheters can cause inflammation or even demyelination in nerve tissue. (Auroy Y et al, 1997). 
It should be noted that not all neurological deficits occurring after a regional anesthetic are the result of the block. Surveys of complications have reported many instances of postoperative neurological deficits that were attributed to regional anesthesia when in fact only general anesthesia was used. Postpartum deficits including lateral femoral cutaneous neuropathy, foot drop, and paraplegia were recognized before the modern era of anesthesia and still occur in the absence of anesthetics. Less clear are the postanesthetic cases complicated by concurrent conditions such as atherosclerosis, diabetes mellitus, intervertebral disk disease, and spinal disorders. (Auroy Y et al, 2002).

Both prospective and retrospective studies have reported major injuries associated with regional anesthesia. (Auroy Y et al, 2002). Cardiac arrest occurring during spinal blockade has been reported to be as high as 6.4 per 10,000 patients, with many of these arrests attributed partially or completely to the spinal anesthetic. (Biboulet $\mathbf{P}$, et al 2001). Paraplegia, cauda equina syndrome, and seizures resulting from unintentional intravenous injections of local anesthetic are other serious complications that have resulted from regional anesthesia. In addition to these major complications, less severe injuries such as post-dural puncture headache and back pain are frequent sequelae of regional anesthesia. Obstetric patients represent a unique group of patients of similar age, American Society of Anesthesiologists (ASA) physical status, and physiology who routinely receive regional anesthetics. Predominately minor complications such as back pain and post-dural puncture headache from neuraxial blockade have been reported in obstetric patients. We used the database of the ASA Closed Claims Project to identify specific patterns of injury and legal liability associated with regional anesthesia, with a subset comparison between obstetric and nonobstetric neuraxial anesthesia claims. A detailed analysis was performed to examine factors associated with high-severity injuries such as death or brain damage, nerve injuries, and neuraxial complications (Schultz A et al, 1996).

The damaging events in almost half of obstetric and nonobstetric neuraxial anesthesia cases in the ASA Closed Claims database were block related. Obstetric neuraxial anesthesia claims had a significantly greater proportion of claims associated with temporary and low-severity injuries compared with nonobstetric claims. Approximately half of high-severity claims in both groups were block-related complications predominately from neuraxial cardiac arrest and unintentional intravenous injection. Permanent nerve injuries such as lumbosacral nerve root injuries and paraplegia accounted for a significantly lower proportion of obstetric neuraxial anesthesia claims compared with nonobstetric claims. Eye injuries, which were predominately block related, comprised 5\% of the regional anesthesia claims and usually resulted in permanent injury (Webb R and Kantor G, 1992).

\section{Limitations of the ASA Closed Claims Database}

The analysis of data collected from the ASA Closed Claims Project has several limitations. The database does not contain claims on all adverse anesthetic events, nor does it have any denominator data on how many anesthetics are performed per year. Consequently, estimates of risk for specific regional anesthesia procedures or populations cannot be made. Other limitations include the nonrandom, retrospective collection of data provided partially by direct participants instead of impartial observers; the bias toward substandard care designations for poor outcomes; and changing anesthetic practice and standards during the 20-yr time span for this data. Despite these limitations, the ASA Closed Claims database provides useful information on large numbers of rare adverse events that are not amenable to prospective study from single centers (Caplan $\mathbf{R}$ et al, 1991).

\section{Neuraxial anesthesia Claims}

\section{[A]Permanent Nerve Injuries:}

The majority of neuraxial complications associated with regional anesthesia claims resulted in permanent neurologic deficits. However, complications caused by meningitis, abscesses, or herniated discs usually demonstrated good recovery. Hematoma was the most common cause of neuraxial injuries, and the majority (72\%) of these cases were associated with either intrinsic or iatrogenic coagulopathy (Vandermeulen et al, 1994).

They found that 42 of 61 neuraxial hematomas (68\%) were associated with impaired coagulation. Intraoperative anticoagulation for vascular surgery was used in more than half of the neuraxial hematoma claims and was combined with other medications that can impair coagulation (aspirin, urokinase, ketorolac, dextran, coumadin, and subcutaneous heparin) either preoperatively or postoperatively in three claims. Low-molecular-weight heparin was used in only 2 cases of neuraxial hematoma, but this small number of cases may reflect the 3- to 5-yr time lag for claims to be settled and entered into the ASA Closed Claims database. Intrinsic coagulopathy was present in only 2 cases (Vandermeulen $\mathbf{E}$ et al, 1994). 
The benefits of regional anesthesia, particularly for peripheral vascular surgery with reduced graft thrombosis and improved graft blood flow, have been described in several reports. .( Christopherson $\mathbf{R}$ et al, 1993). In addition, the low frequency of epidural hematomas in regional anesthesia for vascular surgery with anticoagulation has also been demonstrated in three studies with no epidural hematomas reported in approximately 6,000 patients cumulatively. A risk-benefit assessment of neuraxial anesthesia in an anticoagulated patient should be made preoperatively with appropriate informed consent. Although many studies and texts state that back pain is the cardinal symptom of an epidural hematoma, our data and several other studies demonstrate that an increased motor block out of proportion to the infused local anesthetic is the most common presenting symptom (Wysowski D et al., 1998).

Back pain may be less prominent in the presence of a local anesthetic, opiate infusion, or both. Other symptoms include increased sensory block and bowel and bladder dysfunction. The critical factor for a favorable outcome with epidural hematoma is time to treatment with decompression of the spinal cord. Improved surveillance for this complication, especially in patients who were anticoagulated at any time during a neuraxial anesthetic, and emergent diagnostic workup and treatment may decrease the number of epidural hematoma cases with permanent neurologic injury (Van Aken V and Vermylen J, 1994).

Four cases of epidural/spinal hematoma after subarachnoid block had evidence of needle trauma in the thoracic region, and one case of thoracic epidural hematoma resulted after a labor epidural. Studies demonstrating considerable anatomical variability between patients in the location of the end of the spinal cord (T12-L3), as well as the variability in the iliac crest alignment with lumbar interspaces (L3-L4 to L5-S1), may partially explain some of these injuries. Body habitus may further complicate correct identification of lumbar interspaces. Given these limitations, placement of spinal anesthetics at the lowest interspace possible might decrease the number of spinal cord injuries caused by direct needle trauma (Kim J, Bahk J and Sung J, 2003).

\section{[B]Temporary Nerve Injuries:}

The majority of claims in the obstetric neuraxial anesthesia group were associated with temporary injuries. A higher proportion of minor injuries for headache, back pain, inadequate analgesia, and emotional distress were present in obstetric patients compared with nonobstetric patients. In contrast, a lower proportion of claims were associated with nerve damage in the obstetric group (17\%) compared with the nonobstetric group (36\%). These differences in the types of claims between obstetric and nonobstetric groups may partially be explained by the physiology of young laboring women who are at high risk for post-dural puncture headache and back pain. Moreover, the higher proportion of claims for inadequate analgesia may partially result from the limitations in administering supplemental systemic anesthesia in the obstetric population. The relatively high proportion of claims for minor injuries in the obstetric group may also reflect an "idealized" and unrealistic expectation of the pregnant patient that childbirth is a beautiful experience, compared with the group of nonobstetric patients who primarily receive anesthesia for surgery for pathologic conditions (Howell C et al,2002).

\section{Incidence and etiology of neurologic complications}

A prospective survey in France recently evaluated the incidence and characteristics of serious complications related to regional anesthesia. ${ }^{1}$ A total of 103,730 regional anesthetics, including 40,640 spinal and 30,413 epidural anesthetics, 21,278 peripheral nerve blocks, and 11,229 iv regional anesthetics, were performed over a five-month period. The incidence of cardiac arrest and neurologic complications was significantly higher after spinal anesthesia than other types of regional procedures. Neurologic complications related to the regional anesthetic technique occurred in 34 patients; recovery was complete within three months in 19 of 34 patients. In 12 of 19 cases of radiculopathy after spinal anesthesia, and in all cases of radiculopathy after epidural or peripheral block, needle placement was associated with either paresthesia during needle insertion, or pain with injection. In all cases, the radiculopathy had the same topography as the associated paresthesia. The authors concluded that needle trauma and local anesthetic neurotoxicity were the etiologies of most neurologic complications (Cheney FW et al., 1999).

Cheney et al. examined the American Society of Anesthesiologists Closed Claims database to determine the role of nerve damage in malpractice claims filed against anesthesia care providers. Of the 4,183 claims reviewed, 670 (16\%) were for anesthesia-related nerve injury. The most frequent sites of injury were the ulnar nerve (190 claims), brachial plexus (137 claims), lumbosacral roots (105 claims), or spinal cord (84 claims). Regional anesthesia was more frequently associated with nerve damage claims. Ulnar nerve injuries were more often associated with general anesthesia. However, spinal cord and lumbosacral nerve root injuries having identifiable etiology were associated predominantly with a regional anesthetic technique, and were related to paresthesias during needle or catheter 
placement or pain during injection of local anesthetic. It is also notable that despite intensive medicolegal investigation, a definite mechanism of injury is rarely determined (Cheney FW et al., 1999).

\section{[1] Nerve injury from needle and catheter placement}

Direct needle- or catheter-induced trauma rarely results in permanent or disabling neurologic injury. A recent retrospective study of 4,767 spinal anesthetics noted the presence of a paresthesia during needle placement in 298 (6.3\%) of patients. Importantly, four of the six patients with a persistent paresthesia postoperatively complained of a paresthesia during needle placement, identifying elicitation of a paresthesia as a risk factor for a persistent paresthesia. Currently, it is unknown whether clinicians should abandon the procedure if a paresthesia is elicited (rather than replacing the needle), in an effort to decrease the risk of nerve injury during neuraxial block. (Horlocker TT et al , 1997).

The passage and presence of an indwelling catheter into the subarachnoid or epidural spaces or a peripheral nerve sheath presents an additional source of direct trauma. Laboratory studies have demonstrated demyelination and inflammation adjacent to the catheter tract in both the spinal root and cord of rats following placement of indwelling subarachnoid catheters. The use of a catheter may indirectly contribute to neurologic injury. Poor mixing resulting from very slow injection rates through spinal microcatheters may increase the risk of developing high concentrations of hyperbaric local anesthetics in dependent areas of the spinal canal. This is the presumed mechanism of cauda equina syndrome following continuous spinal anesthesia (Drasner K et al, 1994).

\section{[2] Local anesthetic toxicity}

Although most local anesthetics administered in clinical concentrations and doses do not cause nerve damage, prolonged exposure, high dose and/or high concentrations of local anesthetic solutions may result in permanent neurologic deficits. There is both laboratory and clinical evidence that local anesthetic solutions are potentially neurotoxic and that the neurotoxicity varies among local anesthetic solutions (Hodgson PS et al., 1999). For example, cauda equina syndrome has been reported after single dose and continuous spinal anesthesia, intrathecal injection during intended epidural anesthesia, and repeated intrathecal injection after failed spinal block with lidocaine. Presumably, injection (or reinjection) results in high concentrations of local anesthetic within a restricted area of the intrathecal space and causes neurotoxic injury. Attention to patient positioning, total local anesthetic dose and careful neurologic examination (evaluating for preferential sacral block) will assist in the decision to inject additional local anesthetic in the face of a patchy or failed block (Rigler ML et al., 1991).

Differences in neurotoxicity are dependent on pKa, lipid solubility, protein binding and potency. In histopathologic, electrophysiologic, and neuronal cell models, lidocaine and tetracaine appear to have a greater potential for neurotoxicity than bupivacaine at clinically relevant concentrations. Additives such as epinephrine and bicarbonate may also affect neurotoxicity. Addition of $5 \mu \mathrm{g} \cdot \mathrm{ml}^{-1}$ of epinephrine increases the toxicity of both lidocaine and bupivacaine. The presence of a preexisting neurologic condition may predispose the nerve to the neurotoxic effects of local anesthetics (Kalichman MW and Calcutt NA, 1992).

\section{[3] Transient neurologic symptoms}

Transient neurologic symptoms (TNS) were first formally described in 1993. Multiple laboratory and clinical studies have been performed in an attempt to define the etiology, clinical significance, and risk factors associated with TNS. The incidence has ranged between $0 \%$ and $37 \%$, and is dependent on anesthetic, surgical and, possibly, undefined patient factors. A large multicentre epidemiologic study involving 1863 patients was recently performed to identify potential risk factors for TNS. The incidence of TNS with lidocaine (11.9\%) was significantly higher than that with tetracaine $(1.6 \%)$ or bupivacaine $(1.3 \%)$. The pain was described as severe in $30 \%$ of patients and resolved within a week in over $90 \%$ of cases. Outpatient status, obesity and lithotomy position also increase the risk of TNS for patients who receive lidocaine. This suggests that the risk of TNS is high among outpatients in the lithotomy position (24.3\%) and low for inpatients having surgery in positions other than lithotomy (3.1\%). However, these variables were not risk factors with tetracaine or bupivacaine. The authors also reported that neither gender, age, history of back pain or neurologic disorder, lidocaine dose/concentration, spinal needle/type/size, aperture direction, nor addition of epinephrine increased the risk of TNS (Freedman JM et al,1998).

The clinical significance of TNS is unknown. While many anesthesiologists believe that the reversible radicular pain is on one side of a continuum leading to irreversible cauda equina syndrome, there are currently no data to support this concept. It is important to distinguish between factors associated with serious neurologic complications, such as 
cauda equina syndrome, and transient symptoms when making recommendations for the clinical management of patients. For example, increasing the concentration/dose of lidocaine and adding epinephrine increases the risk of irreversible neurotoxicity, but has little effect on the risk of TNS. Therefore, the clinician must determine the appropriate intrathecal solution, including adjuvants, given the surgical duration and intraoperative position for each individual patient (Freedman JM et al,1998).

\section{[4] Neural ischemia}

The blood supply to the spinal cord is precarious due to the relatively large distances between the radicular vessels. Systemic hypotension or localized vascular insufficiency with or without a spinal anesthetic may produce spinal cord ischemia resulting in flaccid paralysis of the lower extremities, or anterior spinal artery syndrome. The addition of epinephrine or phenylephrine theoretically may produce local cord ischemia. However, most animal studies fail to show a significant decrease in spinal cord blood flow and large studies have failed to identify the use of vasoconstrictors as a risk factor for temporary or permanent deficits (Hodgson PS et al, 1999). Most presumed cases of vasoconstrictor-induced neurologic deficits have been reported as single case reports, often with several other risk factors present (Dahlgren N and Tornebrandt K, 1995).

Peripheral nerves have a dual blood supply consisting of intrinsic endoneural vessels and extrinsic epineural vessels. A reduction or disruption of nerve blood flow may result in neural ischemia. Intraneural injection of volumes as small as 50-100 $\mu 1$ may generate intraneural pressures which exceed capillary perfusion pressure for as long as ten minutes and thus cause neural ischemia. Endoneural hematomas have also been reported after intraneural injection. Epineural blood flow is also responsive to adrenergic stimuli. The use of local anesthetic solutions containing epinephrine theoretically may produce peripheral nerve ischemia, especially in patients with microvascular disease (Kalichman MW and Calcutt NA, 1992).

\section{[5] Hemorrhagic complications (spinal hematoma)}

Although hemorrhagic complications can occur after virtually all regional anesthetic techniques, bleeding into the spinal canal is perhaps the most serious hemorrhagic complication associated with regional anesthesia because the spinal canal is a concealed and nonexpandable space. The reader is referred to The Consensus Statements on Neuraxial Anesthesia and Anticoagulation published by the American Society of Regional Anesthesia for a more detailed discussion on regional anesthetic management of the anticoagulated patient (Liu SS and Mulroy MF, 1998).

The actual incidence of neurologic dysfunction resulting from hemorrhagic complications associated with neuraxial blockade is unknown; however, the incidence cited in the literature is estimated to be less than one in 150,000 epidural and less than one in 220,000 spinal anesthetics (Vandermeulen EP et al, 1994).

The decision to perform neuraxial anesthesia and the timing of catheter removal in a patient receiving anticoagulants perioperatively should be made on an individual basis, weighing the small, though definite risk of spinal hematoma with the benefits of regional anesthesia for a specific patient. It is generally accepted that except in extraordinary circumstances, neuraxial blockade should be avoided in patients who have known coagulopathies, have significant thrombocytopenia, or have received thrombolytic therapy within the previous $24 \mathrm{hr}$ (Rosenquist RW and Brown DL, 1998). The patient's coagulation status should be optimized at the time of spinal or epidural needle/catheter placement, and the level of anticoagulation must be carefully monitored during the period of epidural catheterization. Prolonged therapeutic anticoagulation appears to increase risk of spinal hematoma formation, especially if combined with other anticoagulants or thrombolytics (Liu SS and Mulroy MF, 1998). Indwelling catheters should be not removed in the presence of therapeutic anticoagulation, as this appears to significantly increase the risk of spinal hematoma. Patients should be closely monitored in the perioperative period for early signs of cord compression such as severe back pain, progression of numbness or weakness, and bowel and bladder dysfunction. A delay in diagnosis may lead to irreversible cord ischemia (Horlocker TT and Wedel DJ, 1998).

\section{[6] CSF leak:}

Persistent cerebrospinal fluid (CSF) leak is an apparently rare complication of dural puncture from spinal or epidural anesthesia. Combined spinal-epidural techniques are increasingly popular but persistent CSF leak has not been reported. $\beta_{2}$-transferrin immunofixation assay can be useful as a diagnostic test for the presence of CSF in this situation (Chan OB et al., 2004). 


\section{[7] Infectious complications}

Infection can complicate any regional technique, but are of greatest concern when infection occurs within the spinal canal. The infectious source can be exogenous due to contaminated equipment or medication, or endogenous secondary to a bacterial source in the patient seeding to the remote site of needle or catheter insertion. In addition, indwelling catheters may be colonized from a superficial site and subsequently serve as a wick for spread of infection from the skin to the epidural space (or other neural sheath). An alternative mechanism may be contamination with viridans streptococci from the operator's buccal mucosa (Du Pen SL et al., 1990).

\section{Patients with preexisting neurologic disorders}

Patients with preexisting neurologic disease present a unique challenge to the anesthesiologist. The presence of preexisting deficits, signifying chronic neural compromise, theoretically places these patients at increased risk for further neurologic injury. The presumed mechanism is a "double crush" of the nerve at two locations resulting in a nerve injury of clinical significance. The double crush concept suggests that nerve damage caused by traumatic needle placement/local anesthetic toxicity during the performance of a regional anesthetic may worsen neurologic outcome in the presence of an additional patient factor or surgical injury. Progressive neurologic diseases may also coincidentally worsen perioperatively, independent of the anesthetic method. If a regional anesthetic is indicated or requested, the patient's preoperative neurologic examination should be formally documented and the patient must be made aware of the possible progression of the underlying disease process (Dahlgren $\mathbf{N}$ and Tornebrandt $\mathbf{K}$, 1995).

Regional anesthetic management is based on the theory that patients with preoperative neurologic deficits may undergo further nerve damage more readily from needle or catheter placement, local anesthetic systemic toxicity, and vasopressor-induced neural ischemia. Dilute or less potent local anesthetic solutions should be used when feasible to decrease the risk of local anesthetic toxicity. The potential risk of vasoconstrictor-induced nerve ischemia must be weighed against the advantages of improved quality and duration of block. Because epinephrine and phenylephrine also prolong the block and therefore neural exposure to local anesthetics, the appropriate concentration and dose of local anesthetic solutions must be thoughtfully considered (Dahlgren $\mathbf{N}$ and Tornebrandt K, 1995).

\section{Diagnosis and evaluation of neurologic complications}

It is imperative that all preoperative neurologic deficits are documented to allow early diagnosis of new or worsening neurologic dysfunction postoperatively. Postoperative sensory or motor deficits must also be distinguished from residual (prolonged) local anesthetic effect. Imaging techniques, such as computed tomography (CT) and magnetic resonance imaging (MRI) are useful in identifying infectious and inflammatory processes as well as expanding hematomas (Horlocker TT, 2001).

Although most neurologic complications resolve completely within several days or weeks, significant neural injuries necessitate neurologic consultation to document the degree of involvement and coordinate further work-up. Neurophysiologic testing, such as nerve conduction studies, evoked potentials, and electomyography (EMG) are often useful in establishing a diagnosis and prognosis. A reduced amplitude in evoked responses indicates axonal loss, while increased latency occurs in the presence of demyelination. Fibrillation potentials are present during active axonal degeneration. They appear two to three weeks after injury and are maximal at one to three months. Because of the decreased number of axons present in patients with neurologic conditions, there is a reduction in neuron recruitment during voluntary effort. The degree of reduction parallels the severity of the disorder. Despite many applications, nerve conduction studies have several limitations. Typically only the large sensory and motor nerve fibers are evaluated; dysfunction of small unmyelinated fibers would not be detected. In addition, abnormalities will not be noted on EMG immediately after injury, but rather require several weeks to evolve. Although it is often recommended to wait until evidence of denervation has appeared before performing neurophysiologic testing, a baseline study (including evaluation of the contralateral extremity) would be helpful in ruling out underlying pathology or a preexisting condition (Horlocker TT, 2001).

In conclusion, major complications after regional anesthetic techniques are rare, but can be devastating to the patient and the anesthesiologist. Prevention and management begin during the preoperative visit with a careful evaluation of the patient's medical history and appropriate preoperative discussion of the risks and benefits of the available anesthetic techniques. Efforts should also be made to decrease neural injury in the operating room through careful 
patient positioning. Postoperatively, patients must be followed closely to detect potentially treatable sources of neurologic injury, including hematoma or abscess, constrictive dressings, improperly applied casts, and increased pressure on neurologically vulnerable sites. New neurologic deficits should be evaluated promptly to document formally the patient's evolving neurologic status, arrange further testing or intervention, and provide long-term follow-up (Horlocker TT, 2001).

\section{Neurologic Complications of Peripheral Nerve Blocks INTRODUCTION}

Although there are relatively few published reports of anesthesia-related nerve injury associated with the use of peripheral nerve blocks (PNBs), it is likely that the commonly cited incidence $(0.4 \%)$ of severe injury is underestimated owing to underreporting (Auroy et al, 2002). Most complications of peripheral nerve blocks were reported with upper extremity blocks. The less frequent clinical application of lower-extremity nerve blocks may be the main reason that there are even fewer reports of anesthesia-related nerve injury associated with lower-extremity PNBs as compared with upper-extremity PNBs. While neurologic complications after PNBs can be related to a variety of factors related to the block (e.g., needle trauma, intraneuronal injection, neuronal ischemia, and toxicity of local anesthetics), a search for other common causes should also include positional and surgical factors (eg, positioning, stretching, retractor injury, ischemia, and hematoma formation). In some instances, the neurologic injury may be a result of a combination of these factors (Volka J et al., 1997).

\section{Functional histology of the peripheral nerves:}

The functional histology of the peripheral nerve is important to understand the mechanisms of peripheral nerve injury. A peripheral nerve is a complex structure consisting of fascicles held together by the epineurium-an enveloping, external connective sheath, (Figure II-1). Each fascicle contains many nerve fibers and capillary blood vessels embedded in a loose connective tissue, the endoneurium.I(Sunderland S, 1978). The perineurium is a multilayered epithelial sheath that surrounds individual fascicles and consists of several layers of perineural cells. Therefore, in essence, a fascicle is a group of nerve fibers or a bundle of nerves surrounded by perineurium. Of note, fascicles can be organized in 1 of 3 common arrangements: monofascicular (single, large fascicle); oligofascicular (few fascicles of various sizes); and polyfascicular (many few fascicles of various sizes) (Millesi H and Terzis JK, 1984).

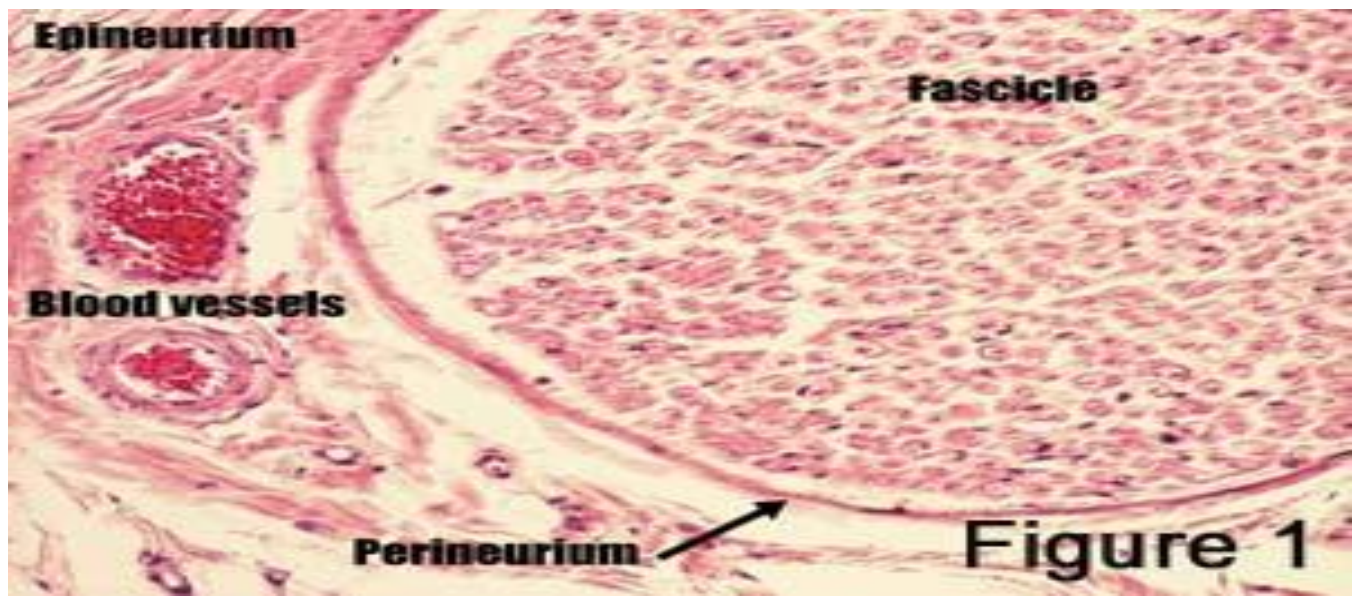

Figure 1. Histology of the peripheral nerve.

Shown are a large fascicle of the peripheral nerve with its axons, surrounded by perineurium, epineurium and nourishing blood vessels.

Nerve fibers can be myelinated or unmyelinated; sensory and motor nerves contain both in a ratio of 4:1, respectively. Unmyelinated fibers are composed of several axons, wrapped by a single Schwann cell. The axons of myelinated nerve fibers are enveloped individually by a single Schwann cell. A thin layer of collagen fibers, the endoneurium, surrounds the individually myelinated or groups of unmyelinated fibers. 
Nerve fibers depend on a specific endoneurial environment for their function. Peripheral nerves are richly supplied by an extensive vascular network in which the endoneurial capillaries have endothelial "tight junctions," a peripheral analogy to the "blood-brain barrier." The neurovascular bed is regulated by the sympathetic nervous system, and its blood flow can be as high as $30-40 \mathrm{~mL} / 100 \mathrm{~g} /$ minute (Selander D, 1988) In addition to conducting nerve impulses, nerve fibers also maintain axonal transport of various functionally important substances, such as proteins, and precursors for receptors and transmitters. This process is highly dependent on oxidative metabolism. Any of these structures and functions can be deranged during a traumatic nerve injury, with the possible result of temporary or permanent impairment or loss of neural function (Selander D, 1988).

The size and the number of the fascicles greatly in a peripheral nerve substantially vary from one peripheral nerve to another. In general, the larger the nerve, the greater the number and the size of the fascicles. Additionally, the larger the fascicle, the greater is the risk of intraneural injection as large fascicles can accommodate the tip of the needle (Sunderland S, 1978). Of note, the fascicular bundles are not continuous throughout the peripheral nerve. They divide and anastomose with one another as frequently, as every few millimeters.9 However, the axons within a small set of adjacent bundles redistribute themselves so that the axons remain in approximately the same quadrant of the nerve for several centimeters. This arrangement is of practical concern to the surgeons trying to repair a severed nerve. If the cut is clean, it may be possible to suture individual fascicular bundles together. In such a scenario, there is a good probability that the distal segment of nerves synapsing with the muscles will be sutured to the central stump of motor axons and the same for sensory axons. In such cases, good functional recovery is possible. If a short segment of the nerve is missing, however, the fascicles in the various quadrants of the stump may no longer correspond with one another, good axial alignment may not be possible and functional recovery is greatly compromised or improbable. This arrangement of the peripheral nerve helps explain why intraneural injections result in disastrous consequences as opposed to clean needle nerve cuts which tend to heal much more readily (Kingsley R, 2000).

The connective tissue of a nerve is tough, compared to the nerve fibers themselves. The connective tissue of a nerve permits a certain amount of stretch without damage to the nerve fibers. The nerve fibers are somewhat "wavy," and when they are stretched, the connective tissue around them is also stretched - giving it some protection. This feature, perhaps, plays a "safety" role in nerve blockade by allowing the nerves to be "pushed" rather than pierced by the advancing needle during nerve localization. For this reason, it is prudent to avoid stretching the nerves and nerve plexii during nerve blockade (such as in axillary brachial plexus block and some approaches to sciatic block) (Sunderland S, 1978).

Nerves receive blood from the adjacent blood vessels running along their course. These feeding branches to larger nerves are of macroscopic size and irregularly arranged, forming anastomoses to become longitudinally running vessel(s) that supply the nerve and give off subsidiary branches. Although the connective tissue sheath enveloping nerves serves to protect the nerves from stretching, it also believed that neuronal injury after nerve blockade may be due, at least partly, to the pressure or stretch within poorly stretchable connective sheaths and the consequent interference with the vascular supply to the nerve (Kingsley R, 2000).

\section{Mechanisms Of Peripheral Nerve Injury}

The etiology of peripheral nerve injury related to the use of PNBs falls in 1 of 4 categories, (Table III-1). Laceration results when the nerve is cut partially or completely, such as by a scalpel or a large-gauge cutting needle. Stretch injuries to the nerves may result when nerves or plexuses are stretched in a nonphysiologic or exaggerated physiologic position, such as during shoulder manipulation under an interscalene block. Pressure, as a mechanism of nerve injury, is relatively common. Typical example of this mechanism is chronic compression of the nerves by neighboring structures, such as fibrous bands, scar tissue, or abnormal muscles where they pass through fibroosseous spaces if the space is too small, such as the carpal tunnel. Such chronic compression syndromes are called entrapment neuropathies. Examples of pressure injuries applicable to PNBs include external pressure over a period of hours (e.g., a "saturday night palsy" resulting from pressure of a chair back on the radial nerve of the insensate arm). The pressure may be repeated and have a cumulative effect (e.g., an ulnar neuropathy resulting from habitually leaning on the elbow). Such a scenario is conceivable, for instance, in a patient who positions the anesthetized arm (e.g., long-acting or continuous brachial plexus block) in a nonphysiologic position for a few hours. Another example of pressure-related nerve injury is prolonged use of a high-pressure tourniquet. Finally, an intraneural injection may lead to sustained high intraneural pressure, which exceeds capillary occlusion pressure, and leads to nerve ischemia (Selander D, 1999). Vascular nerve damage after nerve blocks can occur when there is acute 
occlusion of the arteries from which the vasa nervora are derived or from a hemorrhage within a nerve sheath. With injection injuries, the nerve may be directly impaled and the drug injected directly into the nerve, or the drug may be injected into adjacent tissues, causing an acute inflammatory reaction or chronic fibrosis, both indirectly involving the nerve. Chemical nerve injury is the result of tissue toxicity of injected solutions (e.g., local anesthetic toxicity, neurolysis with alcohol or phenol, etc.) (Selander 1999).

Table III-1. Mechanism of peripheral nerve injury related to peripheral nerve blocks

\begin{tabular}{|lr|}
\hline Mechanical-acute & injection \\
Laceration & ischemia \\
Stretch & \\
Intraneural & Vascular \\
Acute & syndrome \\
Hemorrhage & \\
Pressure & \\
Extraneural & Intraneural \\
Compartment & Chemical \\
Injection of neurotoxic solutions & \\
\hline
\end{tabular}

Clinical Classification of Acute Nerve Injuries:

Classification of acute nerve injuries is useful when considering the physical and functional state of damaged nerves. In his classification, the terms neurapraxia, axonotmesis, and neurotmesis were introduced (Seddon H, 1943). (Table III- 2) Then a 5-grade classification system was subsequently proposed (Sunderland S, 1951).

Neuropraxia refers to nerve dysfunction lasting several hours to 6 months after a blunt injury to the nerve. In neuropraxia, the nerve axons and connective tissue structures remain intact. The nerve dysfunction probably results from several factors, of which focal demyelination is the most important abnormality. Intraneural hemorrhage, changes in the vasa nervora, disruption of the blood-nerve barrier and axon membranes, and electrolyte disturbances all may add to the impairment of nerve function. Because the nerve dysfunction is rarely complete, clinical deficits are partial and recovery usually occurs within a few weeks, although some neurapraxic lesions (with minimal or no axonal degeneration) may take several months to recover (Seddon $\mathbf{H}, \mathbf{1 9 4 3})$.

Axonotmesis consists of physical interruption of the axons but within intact Schwann cell tubes and intact connective tissue structures of the nerve (ie, the endoneurium, perineurium, and epineurium). Sunderland subdivided this group, depending on which of the 3 structures were involved, Table III-2. With axonotmesis, the nerve sheath remains intact, enabling regenerating nerve fibers to find their way into the distal segment. Consequently, efficient axonal regeneration can eventually take place (Seddon H, 1943).

Neurotmesis refers to a complete interruption of the entire nerve including the axons and all connective tissue structures (epineurium included). Clinically, there is total nerve dysfunction. With both axonotmesis and neurotmesis, axonal disruption leads to wallerian degeneration, from which recovery occurs through the slow process of axonal regeneration. However, with neurotmesis, the 2 nerve ends may be completely separated, and the regenerating axons may not be able to find the distal stump. For these reasons, effective recovery does not occur unless the severed ends are sutured or joined by a nerve graft. With closed injuries the only way to distinguish clearly between axonotmesis and neurotmesis is surgical exploration and intraoperative inspection of the nerve (Seddon

H,

1943).

Table III-2. Classification of nerve injuries*

\begin{tabular}{|l|l|l|}
\hline Seddon & Sunderland & Structural and functional processes \\
\hline Neurapraxia & 1 & Myelin damage, conduction slowing, and blocking \\
\hline Axonotmesis & 2 & Loss of axonal continuity, endoneurium intact, no conduction \\
\hline Neurotmesis & 3 & $\begin{array}{l}\text { Loss of axonal and endoneurial continuity, } \\
\text { perineurium intact, no conduction }\end{array}$ \\
\hline
\end{tabular}




\begin{tabular}{|l|l|l|}
\hline & 4 & $\begin{array}{l}\text { Loss of axonal, endoneurial, and perineurial continuity; } \\
\text { epineurium intact; no conduction }\end{array}$ \\
\cline { 2 - 3 } & 5 & Entire nerve trunk separated; no conduction \\
\hline
\end{tabular}
Based on data from Seddon, Sunderland, and Lundborg (Seddon H, 1943 and Sunderland S, 1951 andLundborg G,

It should be noted that most acute nerve injuries are mixed lesions. Different fascicles and nerve fibers typically sustain different degrees of injury, which may make it difficult to assess the type of injury and predict outcome even by electrophysiologic means. Recovery from a mixed lesion is characteristically biphasic; it is relatively rapid for fibers with neurapraxic damage, but much slower for axons that have been totally interrupted and have undergone wallerian degeneration (Seddon H, 1943).

\section{Mechanical nerve injury}

Intraneural Injection

As opposed to a relatively clear injury caused by a sharp needle cut, intraneural injection has the potential to create structural damage to the fascicle(s) that is more extensive and less likely to heal, Figure 2. Indeed, the devastating sequelae of sensory and motor loss after injection of various agents into peripheral nerves has been well documented

(Hudson A et al., 1980).

Nearly all experimental studies on this subject have demonstrated that the site of injection is critical in determining the degree and nature of injury. More specifically, to induce neurologic injury, the injectate must be injected intrafascicularly; extrafascicular injections of the same substance typically do not cause nerve injury. Thus, the main factor leading to a substantial peripheral nerve damage associated with injection techniques is injection of local anesthetic into a fascicle. This causes mechanical destruction of the fascicular architecture and sets into motion a cascade of pathophysiologic changes including inflammation, cellular infiltration, axonal degeneration, and others, all possibly leading to nerve scaring (Mackinnon S and Dellon A, 1988).

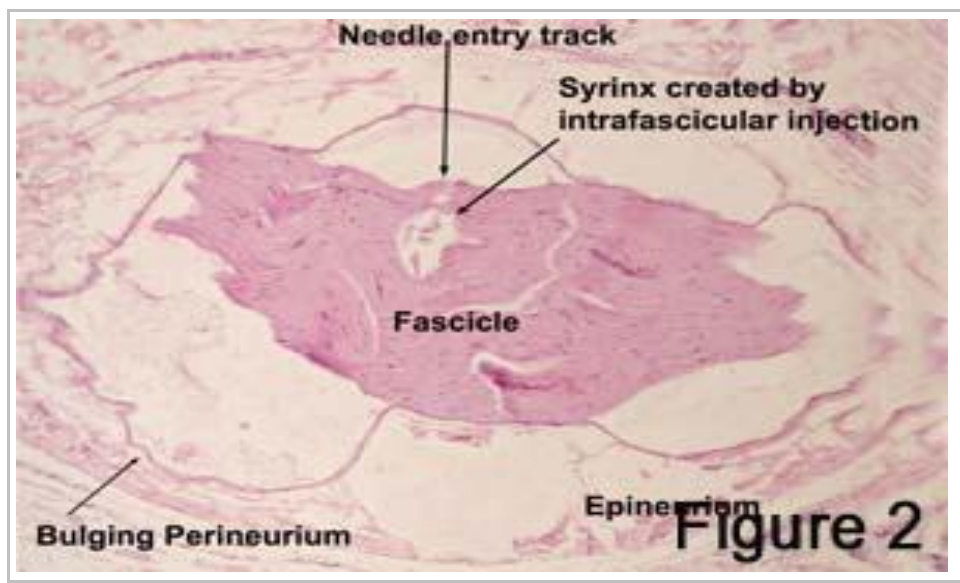

Figure 2. An example of mechanical injection injury to the peripheral nerve.

Shown is a large fascicle, with a needle track,

syrinx created by hydrostatic pressure of the injectate,

as well as the needle track into the fascicle.

Perineurium is seen bulging off the surface of the fascicle.

Histologic features of injury after intraneural injection are rather nonspecific and range from simple mechanical disruption and delamination, to fragmentation of the myelin sheath and marked cellular infiltration, Figure III-3. Using a variety of animal models of nerve injury, a vast array of cellular changes following peripheral nerve trauma have been documented (Mackinnon S and Dellon A, 1988).

The extent of actual neurologic damage after an intrafascicular injection can range from neuropraxia with minimal structural damage to neurotmesis with severe axonal and myelin degeneration, depending upon the needle-nerve relationship, agent injected and dose of the drug used (Strasberg J et al., 1999). In general, subperineural changes 
tend to be more prominent, compared with the central area of the fascicle. Additionally injury to primary sensory neurons which is not detectable hsitologically, causes a shift in membrane channel expression, sensitivity to algogenic substances, neuropeptide production, and intracellular signal transduction, both at the injury site and in the cell body in the dorsal root ganglion. All of this leads to increased excitability and the occurrence of acute or chronic pain often experienced by patients with neurologic injury. It should be noted that intraneural injection and its resultant mechanical injury are merely the inciting mechanisms; a host of additional changes occur involving inflammatory reactions, chemical neuritis, intraneural hemorrhage, all of which eventually leading to nerve scaring and chronic neuropathic pain (Claudio RE et al., 2004).

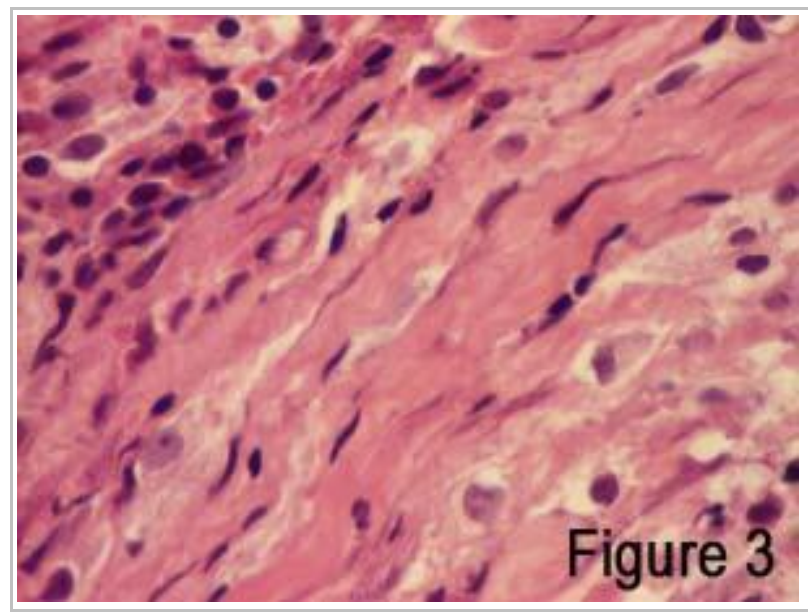

Figure 3. Fascicular injury after an intraneural injection.

Shown is loss axonal degeneration,

extravasation of erythrocytes and inflammatory cell infiltration.

Prevention of Intraneural Injection

Pain on injection

Little is known about how to avoid an intraneuronal injection. Pain with injection has long been thought of as the cardinal sign of intraneuronal injection; consequently, it is commonly suggested that blocks be avoided in heavily premedicated or anesthetized patients. However, numerous case reports have suggested that pain may not be reliable as a sole warning sign of impending nerve injury, and it may present in only a minority of cases (Bhananker $\mathbf{S}$ and Domino K, 2004). However, univariate analysis of potential risk factors for postoperative neurologic dysfunction failed to demonstrate paresthesia as a risk factor (Fenali $\mathbf{G}$ et al., 1999). In addition, the sensory nature of the painparesthesia can be difficult to interpret in clinical practice. For instance, a certain degree of discomfort on injection ("pressure paresthesia") is considered normal and affirmative of impending successful blockade because it is thought that this symptoms indicates that injection of local anesthetic has been made in the vicinity of the targeted nerve. In clinical practice however, it can be difficult to discern when pain-paresthesia on injection is "normal" and when it is the ominous sign of an intraneural injection. Moreover, it is unclear how pain or paresthesia on injection, even when present, can be used clinically to prevent development of neurologic injury (Auroy Y et al, 1997).

\section{Intensity of the stimulating current}

The optimal current intensity resulting in accurate localization of a nerve has been a topic of controversy (Raj P et al., 2002). For instance, stimulation at currents higher than $0.5 \mathrm{~mA}$ may result in block failure because the needle tip is distant from the nerve, whereas stimulation at currents lower than $0.2 \mathrm{~mA}$ theoretically may pose a risk of intraneuronal injection (Voelckel W et al., 2005). Other authors suggest that a motor response with a current intensity between 1.0 and $0.5 \mathrm{~mA}$ is sufficient for accurate placement of the block needle (Raj P et al., 2002), while some advise using a current of much lower intensity (0.5 to $0.1 \mathrm{~mA})($ Jankovic D and Wells C, 2001). Others simply suggest stimulating with currents less than $0.75 \mathrm{~mA}$ (Jankowski CJ et al., 2005), or progressively reducing the current to as low a level as possible while still maintaining a motor response (Chelly J, 1999).

Most recently published reports on nerve blocks have suggested obtaining nerve stimulation with currents of $0.2-0.5$ $\mathrm{mA}(100 \mathrm{msec})$ before injecting local anesthetics, believing that motor response with current intensities lower than 
$0.2 \mathrm{~mA}$ may be associated with intraneural needle placement. However logical these beliefs might sound, there are no published clinical reports substantiating these concerns ( Hadzic A et al., 2007).

Consequently, in current clinical practice, development of nerve localization and injection monitoring techniques to reliably prevent intraneural injection remains elusive (Fremling $M$ and Mackinnon S, 1996). Nerve stimulators are very useful for nerve localization; however, the needle-nerve relationship cannot be adequately precisely and reliably ascertained as early literature suggests (Raj P et al., 2002). Response to nerve stimulation with a commonly used current intensity $(1 \mathrm{~mA})$ may be absent even when the needle makes physical contact with or is inserted into a nerve (Sala-Blanch $X$ et al., 2004). Occurrence of nerve injuries despite using nerve stimulation to localize nerves further suggests that nerve stimulators can at best provide only a rough approximation of the needle-nerve relationship. One fundamental problem with the nerve stimulation is that the current flows in all directions following the path of the least resistance and not necessarily only towards the nerve. Miniscule changes at the needle tip-tissue interface can make a substantial difference on the preferential flow of current away from the nerve. This may results in cessation of the motor response even when needle is in intimate relationship with the nerve or even intraneurally. The current interest for ultrasound-assisted nerve localization holds promise for facilitating nerve localization and administration of nerve blocks; however, the image resolution of this technology is insufficient to visualize nerve fascicles and prevent intrafascicular injection (Auroy Y et al, 2002).

Resistance to injection

Assessing resistance to injection is a common practice, similar to loss of resistance to injection of air or saline using a "syringe feel" during administration of epidural, paravertebral, or lumbar plexus blocks. Similarly, assessing tissue resistance and injection compliance is another means of estimating the anatomic location of the needle tip during the practice of PNBs. For this, clinicians use a "syringe feel" to estimate what may be an abnormal resistance to nerve block injection and thus, reduce the risk of intraneural injection (Weaver M et al., 2002). However, this practice has significant inherent limitations (Claudio RE et al., 2004). For instance, the resistance to injection is greater with smaller needles, introducing additional confusion as to what constitutes "normal" or "abnormal" resistance. Secondly, as opposed to "loss of resistance" in an epidural injection, there is no baseline pressure information or a change in tissue compliance during nerve block injection. In other words, with nerve block injection there is no change in pressure that can be relied upon. For instance, in a study by Claudio and colleagues, all anesthesiologists detected a change in pressure of as little as 0.5 psi during a simulated nerve block injection. However, when gauging the absolute pressure, clinicians substantially varied (by as much as $40 \mathrm{psi}$ ) in their perception of what constituted an abnormal resistance to injection. Finally, no information has been available on what constitutes "normal" and "abnormal" injection pressure during nerve block performance. For these reasons, subjective estimation of resistance to injection is at least as inaccurate as perhaps estimating blood pressure by palpating radial artery pulse; objective means of assessing resistance to injection should be far superior in standardizing injection force and pressure (Claudio RE et al., 2004).

Studies, however, have used clinically more-applicable injection speeds and volumes of local anesthetic in a canine model of nerve injury. The results of these studies suggest that intrafascicular injection is associated with highinjection pressures (> $20 \mathrm{psi}$ ) and carry a risk of neurologic injury, Figures $\mathbf{5}$ and $\mathbf{6}$. Only intraneural injections resulting in pressures greater than $20 \mathrm{psi}$ have been associated with clinically detectable neurologic deficits (Figure 7) as well as histologic evidence of injury to nerve fascicles (Volka J et al., 1997). 


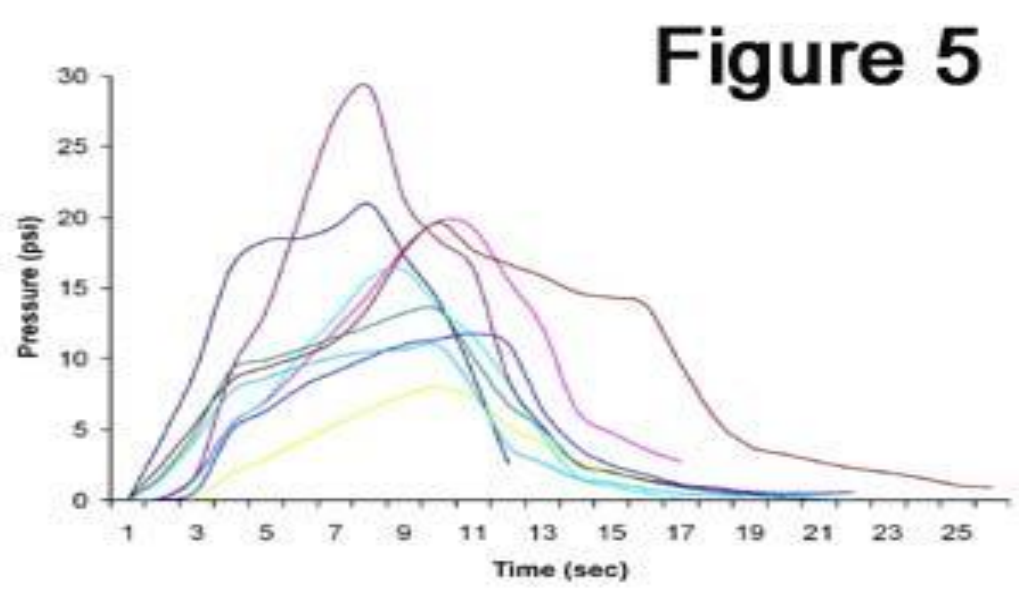

Figure 5. Injection pressures recorded during perineural injection of $2 \%$ lidocaine in a sciatic nerve block model in pigs. Using an injection speed of $15 \mathrm{ml} / \mathrm{min}$ and 25 gauge insulated nerve block needle injections pressures were at or bellow 20 psi in all but one injection.* * Kapur E, et al. 2006 Unpublished data.

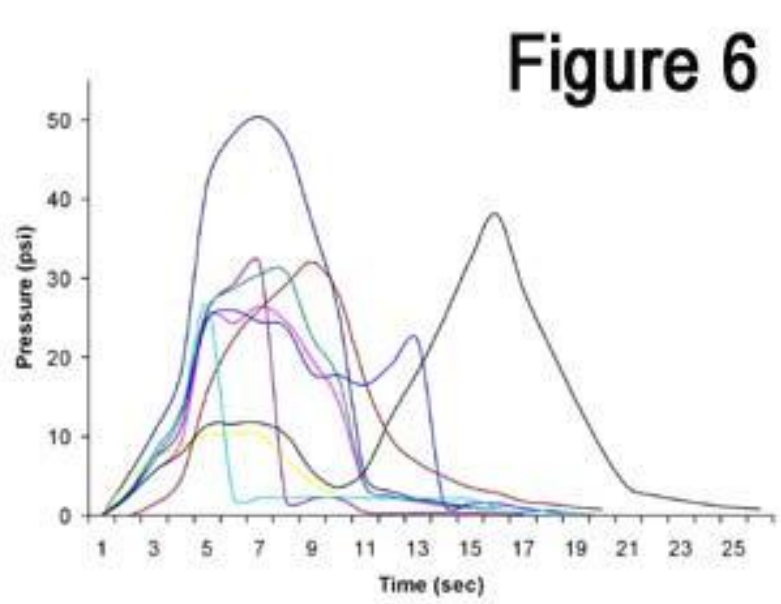

Figure 6. Injection pressures recorded during intraneural injection of $2 \%$ lidocaine in a sciatic nerve block model in pigs. Using an injection speed of $15 \mathrm{ml} / \mathrm{min}$ and 25 gauge insulated nerve block needle injections pressures were at significantly above 20 psi in all but two injections.*

* Kapur E, et al. 2006 Unpublished data.

The current evidence suggests that neurologic injury does not always develop after an intraneural injection (Rice A and McMahon S, 1992). In fact, injection after an intraneural needle placement is more likely to result in deposition of the local anesthetic between and not into the fascicles. Intraneural, but extrafascicular (interfascicular) injection probably occurs more commonly than thought in clinical practice. Such an injection results in a block of unusually fast onset and long duration rather than in a neurologic injury. This is because an intraneural but extrafascicular injection leads to intimate exposure of nerve fascicles to high concentration and doses of local anesthetics. However, permanent neurologic injury does not develop since the local anesthetic is deposited outside the fascicles and the blocks slowly resolve after the injection without evidence of histologic derangement (Sala-Blanch X et al., 2004).

Needle design and direct needle trauma

Needle tip design and risk of neurologic injury have been matters of considerable debate for more than 3 decades. Nearly 30 years ago, Selander and colleagues, suggested that the risk of perforating a nerve fascicle was significantly lower when a short-bevel (e.g., $45^{\circ}$ ) needle was used as opposed to a long-bevel $\left(12^{\circ}-15^{\circ}\right)$ needle. The results of their work is largely responsible for the currently prevalent trend of using short-bevel needles (i.e., angles 
$30^{\circ}-45^{\circ}$ ) for the majority of major peripheral nerve conduction blocks (Selander D et al., 1977). However, the more recent work of Rice and McMahon suggested that when placed intraneurally, short-beveled needles cause more mechanical damage than the long-beveled needles. In their experiment in a rat model, deliberate penetration of the largest fascicle of the sciatic nerve with $12^{\circ}$ - to $27^{\circ}$-beveled needles, with short-beveled needles resulted in the greatest degree of neural trauma. Their work suggests that sharp needles produce clean, more-likely-to-heal cuts, whereas blunt needles produced noncongruent cuts and more extensive damage on the microscopic images. In addition, the cuts produced by the sharper needles were more likely to recover faster and more completely than were the irregular, more traumatic injuries caused by the blunter, short-beveled needles. Although the data on needle design and nerve injury have not been clinically substantiated, the theoretical advantage of short-beveled needles in reducing the risk of nerve penetration has influenced both practitioners and needle manufacturers. Consequently, whenever practical, most clinicians today prefer to use short-beveled needles for major conduction blocks of the peripheral nerves and plexuses. Sharp bevel, small-gauge needles however, continue to be used routinely for many nerve block procedures, such as axillary transarterial brachial plexus block, wrist and ankle blocks, cutaneous nerve block, and others (Rice A and McMahon S, 1992).

Regardless of the considerations related to the needle design and risk of nerve injury, the actual clinical significance of isolated, direct needle trauma remains unclear. For instance, it is possible that both paresthesia and nerve stimulation techniques of nerve localization may often result in unrecognized intraneural needle placement, yet the risk of neurologic injury remains relatively low. Similarly, during femoral arterial cannulation (arterial line insertion), it is likely that the needle is often inadvertently inserted into the femoral nerve, yet injuries to the femoral nerve are rare, and when they occur, they are usually attributed to hematoma formation rather than needle injury (Kent $\mathrm{K}$ et al., 1994). It is possible that a needle-related trauma without accompanying intraneural injection results in injury of a relatively minor magnitude, which readily heals and may go clinically undetected. In contrast, needle trauma coupled with injection of local anesthetic into the nerve fascicles carry a risk of much more severe injury (Claudio RE et al., 2004).

Toxicity of injected solution

Nerves can be injured by direct contact with a needle, injection of a drug into or around the nerve, pressure from a hematoma, or scarring around the nerve (Napiontek M and Ruszkowski K, 1993). Experimental studies have shown that the degree of nerve damage following an injection depends on the exact site of the injection and the type and quantity of the drug used (Gentili $\mathbf{F}$ et al., 1980). The most severe damage is produced by intrafascicular injections, although extrafascicular (subepineurial) injections of some particularly noxious drugs can also produce nerve damage (Mackinnon S et al., 1984). Benzylpenicillin, diazepam, and paraldehyde are the most damaging; however, a number of other medications such as, antibiotics, analgesics, sedatives, and antiemetic medications are also capable of damaging peripheral nerves when injected experimentally or accidentally (Gentili F et al., 1980).

Local anesthetics produce a variety of cytotoxic effects in cell cultures, including inhibition of cell growth, motility, and survival, as well as morphologic changes. The extent of these effects is proportionate to the length of time the cells are exposed to the local anesthetic solution and occur using local anesthetic at normal clinical concentrations. Within normal ranges, the cytotoxic changes are greater as concentrations increase. In the clinical setting, the exact site of local anesthetic deposition plays a critical role in determining the pathogenic potential (Selander D, 1993). After applying local anesthetics outside a fascicle, the regulatory function of the perineural and endothelial bloodnerve barrier is only minimally compromised. High concentrations of extrafascicular anesthetics may produce axonal injury independent of edema formation and elevated endoneural fluid pressure. As with the effects of local anesthetics in cell cultures, the duration of exposure and concentration of local anesthetic determine the degree andincidence of local-anesthetic-induced residual paralysis (Kaneko $S$ et al., 2005).

Neurologic complications following regional anesthesia may also be caused by the direct effects of local anesthetics on the nervous tissue. Toxicity has been reported primarily with the intrathecal use of local anesthetics, but with the increasing popularity of peripheral nerve block anesthesia, reports are surfacing about the direct toxic effects of local anesthetics on peripheral nerves. Several theories regarding the mechanism of injury have been suggested. Prolonged exposure, high doses, high concentrations, body positioning and the specific agent used may cause transient or permanent neurologic injury by a number of intracellular mechanisms. Once the neurologic injury has occurred, it has been suggested that additives such as epinephrine, or the existence of a pre-existing neurologic condition may predispose the patient to neurotoxic effects of local anesthetics (the "double-crush" concept) (Auroy Y et al, 1997). 
Experimental models of neurotoxicity of local anesthetics have included application of local anesthetic to the sciatic nerve in animals, desheathed nerve preparations, and dorsal root ganglion cells in culture using concentration of local anesthetic comparable to those used clinically (Johnson M et al., 2004). These studies have revealed considerable information about the mechanism of injury. Sakura and colleagues discovered that the mechanism did not involve voltage dependent sodium channels. They substituted tetrodotoxin for lidocaine and found that tetrodotoxin blocked these channels as effectively as lidocaine without producing the toxicity associated with lidocaine (Sakura S et al., 1995). Johnson and colleagues discovered that cell toxicity may be related to mitochondrial degradation. Local anesthetics caused the mitochondria to depolarize and stop producing ATP. With the loss of ATP, energy-dependent mechanisms are compromised, leading to the accumulation of calcium intracellularly and activation of enzymes that cause cell degredation. He found that this was unrelated to hypoxia, because lidocaine actually reduced oxygen demand. Cell death or apoptosis was related to the concentration and/or the length of exposure. 1\% lidocaine at an exposure for more than 90 minutes was required to kill $50 \%$ of the cells. Exposures of less than an hour were completely reversible, but exposure to lidocaine at 5\% concentration caused immediate cell death or necrosis (Johnson M et al., 2002).

In addition to electrolyte imbalance (leading to cell death), the loss of ATP has been found to cause failure of axonal transport compromising the ability of the neuron to transport materials synthesized in the perikaryon to the axon terminal (Kanai A et al., 2001).

Fast axonal transport moves neurotransmitters from the cell body to the nerve terminal. Lidocaine has been shown to produce a reversible blockade of rapid axonal transport. Recovery is dependent on the concentration and the exposure time of the local anesthetic on the nerve tissue. High concentrations and/or prolonged exposure has been postulated to cause prolonged or permanent nerve injury (Fagiolini M et al., 1997). Furthermore, the loss of ATP leads to the failure of the sequestration of neurotransmitters within the cells, leading to an increase in the extracellular concentration of glutamate (Kanai A et al., 2001).

Excessive glutamate in the extracellular space through NMDA receptors can exacerbate the elevation of calcium within the cells ultimately leading to further cell degredation. This effect is noted only in the central neuraxis where glutamate is found (Ohtake K, 2000).

Local anesthetics have been shown to cause membrane solubilization at high concentrations. At clinical concentrations, they can form micelles that may act as detergents to disrupt the cell membrane, although this has not been proven in nerve cell membranes (Kitagawa $\mathbf{N}$ et al., 2004). Oda and colleagues demonstrated that $5 \%$ lidocaine and $0.5 \%$ dibucaine were minimum concentrations causing irreversible neurologic damage. No neurologic damage was seen with $2 \%$ lidocaine or $0.2 \%$ dibucaine (Oda M et al., 1998).

Neurotoxicity varies with the local anesthetic solution. In histopathologic, electrophysiologic, and neuronal cell models, lidocaine and tetracaine have been shown to have a greater potential for neurotoxicity than bupivacaine (Hodgson $P$ et al., 1999). Additives, i.e. epinephrine, can increase the toxicity of both lidocaine and bupivacaine. A preexisting neurologic condition, i.e. peripheral neuropathy, injury, surgery, may predispose the patient to nerve injury from toxicity at clinical doses (i.e. double crush concept) (Lynch $\mathbf{N}$ et al., 1996).

In summary, local anesthetics have potentially cytotoxic effects. The mechanisms appear to involve disruption of mitochondrial function, electrolyte imbalance leading to detrimental intracellular calcium accumulation, loss of axonal transport and release of glutamate. The toxicity and ultimate damage to nerve tissue is related to concentration of the agent, site of action, time of exposure and the specific local anesthetic agent used. Most studies have demonstrated a greater effect on the intrathecal use compared to epidural or peripheral nerve exposure. This may be reflect the typically higher baricity, more concentrated dose of local anesthetic bathing the spinal cord for a prolonged period of time as compared to a large volume, less concentrated solution typically used in epidural and peripheral nerve anesthesia (Hadzic A et al., 2007).

Neuronal ischemia 
Lack of blood flow to the primary afferent neuron results in metabolic stress. The earliest response of the peripheral sensory neuron to ischemia is depolarization and generation of spontaneous activity, symptomatically perceived as paresthesias. This is followed by blockade of slow-conducting myelinated fibers and eventually all neurons, possibly through accumulation of excess intracellular calcium, which accounts for the loss of sensation with initiation of limb ischemia. Nerve function returns within 6 hours if ischemic times are less than 2 hours. Ischemic periods of up to 6 hours may not produce permanent structural changes in nerves. However, detailed pathological examination after ischemia initially shows minimal changes, but with 3 hours or more of reperfusion, there develops edema and fiber degeneration that last for 1 to 2 weeks, followed by a phase of regeneration lasting 6 weeks. In addition to neuronal damage, oxidative injury associated with ischemia and reperfusion also affects the Schwann cells, initiating apoptosis (Selander D, 1999).

The perineurium is a tough and resistant tissue layer. An injection into this compartment or a fascicle can cause a prolonged increase in endoneurial pressure, exceeding the capillary perfusion pressure. This pressure, in turn, can result in endoneural ischemia (Selander D, 1999). The addition of vasoconstricting agents theoretically can enhance ischemia because of the resultant vasoconstriction and reduction in blood flow. The addition of epinephrine has been shown in vitro to decrease the blood supply to intact nerves in the rabbit. However, in patients undergoing lowerextremity surgery, addition of epinephrine to the local anesthetic solution used in combined femoral and sciatic nerve blocks has not been shown to be a risk factor for developing postblock nerve dysfunction (Fanelli $\mathbf{G}$ et al., 1999).

Tourniquet Neuropathy

Tourniquet-induced neuropathy is well documented in the orthopedic literature and ranges from mild neuropraxia to permanent neurologic injury (Sharrock $\mathbf{N}$ and Savarese J, 2000). The incidence of tourniquet paralysis has been reported as 1 in 8000 operations (Jankowski C et al., 2003). A prospective study of lower-extremity nerve blockade suggests that higher tourniquet inflation pressure $(>400 \mathrm{~mm} \mathrm{Hg}$ ) was associated with an increased risk of transient nerve injury. 3 Current recommendations for appropriate use of the tourniquet include the maintenance of a pressure of no more than $150 \mathrm{~mm} \mathrm{Hg}$ greater than the systolic blood pressure and deflation of the tourniquet every 90 to 120 minutes (Sharrock $\mathbf{N}$ and Savarese J, 2000). Even with these recommendations, posttourniquet-application neuropraxia may occur, particularly in the setting of preexisting neuropathy (Lichtenfeld N, 1992).

\section{Compressive Hematoma}

Little data exists regarding the safety of PNB in patients treated with anticoagulants. Compressive hematoma formation leading to neuropathy has been associated with needle misadventures when performing lower extremity PNB, particulary with concomitant treatment with anticoagulants (Adam F et al., 2003). However, as opposed to spinal or epidural hematoma, peripheral neuropathy from this etiology typically resolves completely. Regardless, these reports emphasize the important differences in the risk-benefit ratio of PNBs compared with neuraxial blocks in patients receiving anticoagulant therapy (Weller $\mathbf{R}$ et al., 2003).

\section{Peripheral Nerve Blocks In Anesthetized Patients}

Regional anesthesia-associated nerve injury is a significant source of concern for patients, surgeons, and anesthesiologists alike. In addition nerve injury is a potential medico-legal liability for anesthesiologists. Peripheral nerve blocks (PNBs), in particular, are of significant concern because the typical technique involves placing the needle tip in the immediate vicinity of the nerve or plexus. Consequently, any postoperative neurological impairment is automatically, and often unjustly, attributed to the PNB procedure (Jankowski CJ et al., 2003)

Few issues in regional anesthesia have been the subject of as intense controversy as to whether peripheral nerve blocks (PNBs) carry a higher risk of neurological complications when performed in anesthetized patients versus awake patients. Opinions vary from heavy premedication being essential to the success of regional anesthesia63 to its being equated with negligence. Unfortunately, no large-scale controlled studies of the safety of PNBs in awake versus anesthetized patients exists, nor are such studies likely to be available in the future. In the absence of randomized, controlled studies, experts are left to draw conclusions and make logical recommendations solely on their interpretation of the few available case reports, and anecdotal experiences. However, any such recommendations regarding the use of sedation or general anesthesia in patients receiving PNBs could have significant medicolegal repercussions (Hogson P et al., 1999).

\section{SYMPTOMS OF INTRANEURAL INJECTIONS}


The premise behind the common recommendation that PNB should only be performed in awake patients is that an awake patient can provide information that will prevent intraneural injection and therefore avoid neurological injury. This is because it is believed that intraneural injections are excruciatingly painful, and an awake protesting patient is the best available monitor. However, there are three significant problems with this logic (Auroy Y et al, 2002).

(i) The first is that the literature does not support the widespread notion that relying on an a fully awake patient's report of pain on injection is reliable method to prevent nerve injury. In fact, most neurologic complications reported in the literature have not been associated with pain on injection (Shah $\mathbf{S}$ et al., 2005). For instance, of 49 cases of nerve injury found in the literature search (TABLE 3), 48 patients (98\%) were awake. Of these, 42 cases included specific information about the patient's response to the injection; only $4(10 \%)$ patients reported pain on injection. Some reports specifically state that the patient did not have pain, whereas in most others the authors commented that the block performance was uneventful. Interestingly, the pain on administration of local anesthetic (LA) into nerve tissue may be absent even in the central neuraxial area. For instance Kao et al. reported a case of neural injury that was clearly related to spinal cord trauma from a thoracic catheter that had been inserted while the patient was anesthetized; the patient did not have pain during administration of LA postoperatively.84 More recently, Tripathi et al reported a case report of paraplegia after an intracordal injection during attempted steroid injection85, whereas Tsui and Armstrong reported a case of direct spinal cord injury after epidural injection. Both complications occurred in awake patients who did not report pain on needle placement and consequent injection. These report clearly indicate that reliance on pain as a symptom of injection into neurologic tissue is unreliable (Tripathi M et al., 2005).

(ii) The second problem refers to the value of the pain (if present) as monitor in preventing nerve injury, because in cases in which the pain does accompany an intraneural injection it may already be too late to prevent neurologic injury. For example, in the ASA closed-claims study, Cheney and coworkers indicate that on those occasions where pain did occur during injection, the anesthesiologist stopped the injection, however, the patients still went on to develop nerve injury (Cheney F et al., 1999). Similarly, studies utilizing animal models of intraneural injection suggest that nerve fascicles become injured at the very onset of the injection and with injection of very small volume of local anesthetic (as little as $0.5-1 \mathrm{ml}$ ). Thereafter, as injection continues, the fascicle ruptures and the injectate simply leaks out through the ruptured perineurium into the epineurial sheath. At this stage however, the damage to the fascicle(s) may already have been done (Borgeat A et al., 2001).

(iii) Third, pain is notoriously difficult to assess in terms of quality and intensity. Therefore, distinguishing between the discomfort that is commonly seen during local anesthetic injection (which is considered normal) and that of intraneural injection can be difficult (Borgeat $\mathbf{A}$ et al., 2001).

\section{Normal Versus Abnormal Discomfort/Pain On Injection}

Injection of LA in the close proximity to the nerves is often associated with discomfort on injection (KoscielniakNielsen $\mathbf{Z}$ et al., 2004). This is thought to result from "spraying" of the LA in the vicinity of the components of the brachial plexus. Based on his studies of brachial plexus anesthesia, Winnie coined the term "pressure paresthesia" to describe the discomfort patients feel during local anesthetic injection and implied that this was a desirable sign of impending successful blockade (Winnie A, 1970). In actual clinical practice however, the variability of patients' pain thresholds, their ability to verbalize a sensation pain during a procedure, and an anesthesiologist's subjective interpretation of any such response make it very difficult to recommend where a "line" could be drawn between normal and abnormal pain or paresthesia on injection. In fact, a number of published case reports demonstrate that patients' complaints of pain during PNB may not be helpful in preventing the development of the neurological complication. For instance, Barutell et al published a report where a patient communicated discomfort on injection which was perceived as "normal pressure paresthesia" by the anesthesia team and when the injection was carried out; the patient went on to develop permanent neurologic damage (Barutell et al., 1980). Similarly, in the report by Kaufman et al, all 7 patients had discomfort at some point during block injection; however this information could not be used to prevent the development of permanent neurologic injury which occurred in all patients. This however may be an example of case-report bias. In other words, it is possible that patients' reports of pain may have prevented injuries but such events are unlikely to get reported (Kaufman B et al., 2000).

\section{DOGMAS ON COMPLICATIONS OF REGIONAL ANESTHESIA}


Blanket statements and dogmas are common in the field of medicine and regional anesthesia in particular. Much too often, a wide range of recommendations based on a single observation are inappropriately extrapolated. As an example, Walton et al. reported the occurrence of brachial plexus palsy after total shoulder arthroplasty under an otherwise uneventful interscalene block. However, the authors went on to suggest that, "to minimize the risk of brachial plexus injury with interscalene block" PNBs should not be performed in anesthetized patients, and if paresthesia of "unusual severity" occurs, the injection should be immediately stopped. Ironically, their patient was neither anesthetized prior to the block injection, nor did he have paresthesia or pain on injection ( Walton et al., 2000).

Benumof reported 4 cases of severe neurological injury that resulted in cervical paraplegia in patients receiving interscalene brachial plexus blocks under GA (Benumof J, 2000). The discussions that followed however, often recommended that sedation and GA be abandoned to decrease the risk of nerve injury. Such recommendations are based on this case report are inappropriate because none of the patients in this report suffered a peripheral nerve injury. Rather, these patients received an intracordal injection, a complication entirely avoidable with restriction of the needle insertion depth and/or use of more lateral approach to interscalene block (Koscielniak-Nielsen $\mathbf{Z}$ et al., 2004).

Those who base their criticism of "heavy" premedication or GA prior to performing peripheral nerve blocks on the cases reported by Benumof forget that interscalene block is a superficial procedure, devoid of significant discomfort where excessive sedation and analgesia are usually unnecessary except in children who otherwise would not hold still during the procedure. In contrast, many other PNB procedures involve deeper placement of the needle and several attempts at nerve localization that result in significant patient discomfort (Borgeat $\mathbf{A}$ et al., 2003). Therefore, generalized recommendations to avoid premedication during PNBs carry the risk of limiting the use of PNBs because of an inevitable decrease in patient acceptance (Hadzic A et al., 2007).

No study has compared the risk of neurologic complications in awake versus anesthetized patients, and it is unlikely that such studies will ever be done. A review of published reports of injury after PNBs indicates that significant neurologic injury after PNBs in awake patients occurs at a rate of $0.2 \%-0.4 \%$. Most of these reports included brachial plexus blocks only, probably because these techniques are used more frequently than lower extremity nerve blocks (Claudio RE et al., 2004).

In a recent similar prospective evaluation by Bogdanov and Loveland, none of 548 patients who received an interscalene brachial plexus block after induction of GA developed permanent or long-term neurologic complications (Bogdanov A and Loveland R, 2005).

Recent studies indicate that the risk of complications of PNBs after GA may not be substantially more common than complications reported in other similarly powered studies in awake patients. In fact, performance of PNBs in heavily premedicated patients or after induction of GA is undoubtedly a common practice, and a routine in the pediatric anesthesia practice. A recent discussion conducted during the ASRA 2005 session on complications of PNBs indicated that approximately half of the present attendees performed blocks in heavily sedated or anesthetized patients (Hadzic A et al., 2007).

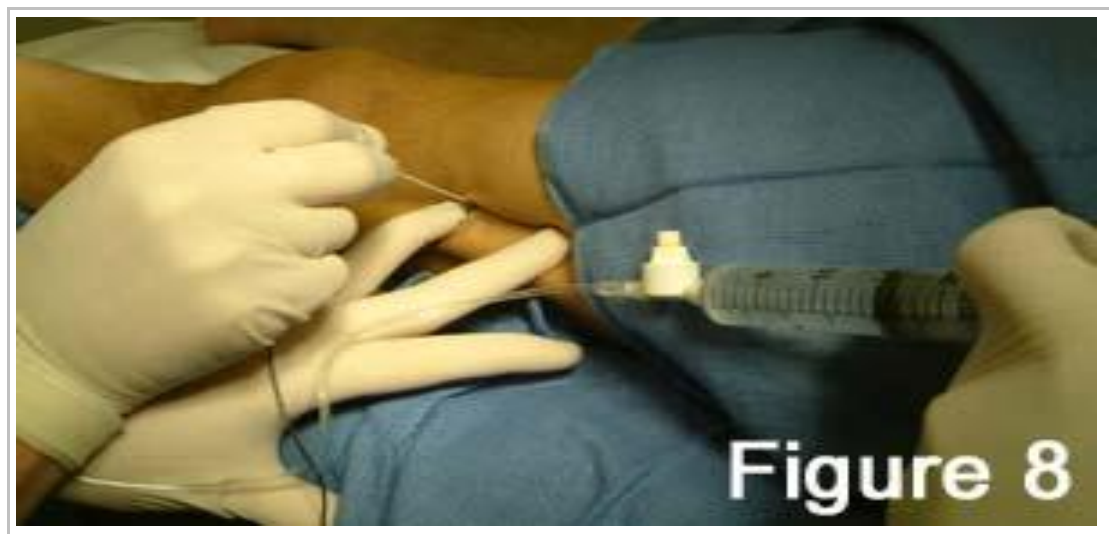


Figure 8. Injection of local anesthetic in lateral approach to popliteal block with in-line monitoring of the injection pressure to avoid pressures $>20$ psi which may be associated with intraneural injection.

RESCUE BLOCKS, MULTIPLE INJECTION TECHNIQUES, AND "REVERSE" AXIS BLOCKS Several PNB techniques that are equivalent of a PNBs in anesthetized patients are accepted as sound practice. These include "rescue" blocks, multiple injection techniques and "reverse" axis blocks; they are all similar to the practice of PNBs in anesthetized patients because the PNB is performed in a partially or fully anesthetized limb.

"Rescue blocks"

Missed nerve blocks may occur from 3\% to $30 \%$ of the time and usually involve only one or two of the terminal nerves.20 Several well established and universally accepted techniques of PNBs, such as repetition of the block, peripheral injection of the nerve and others are often used to rescue failed blocks despite the risk that the needle may be inserted or injection be made into an anesthetized nerve.81 Common examples of rescue blocks after failed axillary or interscalene brachial plexus blocks include elbow and wrist blocks (Mulroy F, 2002).

"Multiple injection techniques"

Multiple injection techniques for both upper and lower limb blockade have been introduced relatively recently in clinical practice with the suggestion that they decrease onset time, increase the success rate, and decreases the required dose of LA. The withdrawal and redirection of the needle to elicit multiple motor responses however, carry a greater risk of direct needle trauma and intraneural injection into already anesthetized nerves. Regardless, this technique has been uniformly accepted by most experts in the field (Koscielniak-Nielsen $Z$ et al., 1997).

\section{"Double blocks" and "repeat" blocks after failed blocks}

Regional anesthesia for elbow surgery has traditionally been a challenge. The most commonly used brachial plexus blocks--the classical approach to interscalene block and the axillary block--are not ideal because they either do not result in reliable block of the ulnar nerve or do not result in adequate analgesia for the tourniquet, respectively. For that reason, in patients undergoing elbow surgery successful regional anesthesia requires the concomitant use of two separate approaches, an interscalene and an axillary approach. Obviously, performance of brachial plexus block at either level precludes the sensory or motor feedback information during performance of the block at the second level (Fanelli G et al., 2001).

\section{AWAKE PATIENTS WILL HAVE SIGNS OF CNS TOXICITY AS A MONITOR BEFORE CVS TOXICITY \\ ENSUES}

Practice of PNBs involves administering large volumes and doses of LAs. A typical clinical presentation of LA toxicity is in an awake or sedated patient during or immediately after injection of LA, followed by sudden onset of confusion, seizure, arrhythmias or cardiac arrest (Mahli A et al., 2002). It has been suggested that heavy sedation or GA increases the risk of severe systemic toxicity of LAs because it diminishes the patient's ability to report early signs and symptoms of rising LA serum levels. However, there are no reports of LA toxicity in adult patients under GA; essentially all reports of toxicity were in awake patients (Ould-Ahmed M et al., 2002). For example, Edde and Deutsch reported an occurrence of cardiac arrest after interscalene brachial plexus block in an awake patient who had no symptoms of toxicity until the entire dose $(20 \mathrm{~mL}$ of $0.5 \%$ bupivacaine) was administered (Edde $\mathbf{R}$ and Deutsch S, 1977). Conversely, others may argue that premedication offers protection because of its anticonvulsive effects. Moreover, since the critical steps in treating patients with severe toxicity is establishment of patent airway, hyperventilation, administration of oxygen, and hemodynamic support, one can argue that anesthetized and ventilated patients who develop systemic toxicity may actually be better off because they already have a secured airway, they are receiving a high concentration of oxygen and they are typically in, an environment that is ideally suited for aggressive resuscitation.

Bernards et al. reported that pigs given an intravenous infusion of bupivacaine failed to demonstrate signs of CNS toxicity prior to cardiovascular collapse if they had been pre-medicated with benzodiazepines (Bernards $\mathbf{C}$ et al., 1989). Indeed, in clinical studies of local anesthetic toxicity, volunteers clearly report an escalating series of symptoms as plasma concentration of local anesthetic rises during continuous, slow intravenous infusion. However, the occurrence of symptoms of local anesthetic toxicity depends on the rate at which drug is injected. The difficulty in extrapolating this into clinical practice is that local anesthetic for the purpose of neuronal blockade is given as a relatively rapid bolus, rather than a slow, escalating continuous infusion as is the case in animal models (Hadzic A et al., 2007). 
For these reason, any suggestion that GA predisposes to a greater risk of severe systemic toxicity of LA is purely theoretical, as there are no data to firmly support this belief. Finally, LA toxicity is of potentially greater concern in the pediatric patient due to lower dose requirements and need for stringent adherence to $\mathrm{mg} / \mathrm{kg}$ dosage rather than a volume dosage as is the common practice in adult anesthesia. Regardless, the practice of regional anesthesia in anesthetized patients is universally accepted in this patient population (Hadzic A et al., 2007).

\section{NERVE BLOCKS IN ANESTHETIZED CHILDREN VERSUS ADULTS}

As opposed to PNBs in adults, performing blocks in anesthetized pediatric patients is a universally accepted practice. This is by necessity, because pediatric patients are unlikely to be cooperative during needle insertion, nerve stimulation and manipulation necessary to accomplish PNB. In addition, most pediatric patients require concomitant administration of GA to allow for immobility during the surgical procedure. However, from the standpoint of risk of complications, this divergence in consensus on PNBs after GA between adults and pediatric patients does not make much sense. In other words, with perhaps the exception of infants, there are no sufficient anatomical, or neurophysiological differences to justify this divergence in recommendations. While one can argue that complications of PNBs in children are rare, PNBs are not routinely used in the pediatric population and there are simply no series comparable to those in adults to allow one to draw a clear conclusion regarding the risk of nerve injury in children. However, a large prospective study performed in France in children demonstrated a small risk of complications with peripheral nerve blocks (Wedel D et al., 1991).

\section{MONITORING POSSIBILITIES DURING PNBs}

Since this discussion focuses on the impact of heavy sedation or GA on the risk of neurologic complications, it is important to discuss the monitoring that is available to reduce the risk of nerve injury during PNBs. In general, there are two phases amenable to monitoring during placement of PNBs. These include needle placement guidance (percutaneous stimulation, ultrasound) and avoidance of intraneural injection (report of pain on injection by the patient (when present), nerve stimulation, and assessment of resistance to injection). With regards to intraneural injection, neither percutaneous stimulation or ultrasound guidance are helpful. Percutaneous stimulation may be helpful with approximating the needle insertion site but it is useless in estimating the needle-nerve relationship. Ultrasound on the other hand, offers real-time needle guidance below the skin level. However, in addition to the required skill, expense and inconvenience of the equipment, the image resolution is simply insufficient to rule out intraneural needle placement. Nerve stimulator-assisted PNBs entered the practice of regional anesthesia with the promise of decreasing the risk of neurologic complications associated with paresthesia technique. However, it soon became apparent that nerve stimulators could not prevent neurologic injury. More recently, it has been suggested that in many circumstances, the motor response to nerve stimulation may be absent at the point at which the needle makes contact with the nerve, and that intraneural needle placement is possible despite the use of nerve stimulators (Gerancher J et al., 2005).

In summary, few issues in the practice of regional anesthesia have evoked such strong and divergent opinion among clinicians as performance of PNBs in anesthetized or deeply sedated patients. This is because administration of PNBs has traditionally been based on individual preferences, clinical impressions, and other subjective criteria, rather then on established practice standards. Avoidance of deep sedation and GA is often suggested to decrease the risk of peripheral nerve injury with PNBs, however, there is no evidence in the literature to suggest that either practice is safer with regards to the risk of nerve injury. Regardless, the serious nature of complications resulting from inadvertent injections into the spinal cord suggests that regional anesthesia techniques close to the centroneuraxis should be practiced with extreme caution and insight into the appropriate depth of the needle insertion whether the patient is awake, sedated or anesthetized. It is the opinion of these authors that proper training, and use of proper techniques and nerve block/monitoring equipment are more likely to decrease the risk of complication than are unfounded blanket statements as to the advisability of performing peripheral nerve blocks in anesthetized patients. This is because such statements are unfounded by the relevant literature and can have a potentially negative impact on the practice of regional anesthesia. Many patients and PNB techniques require appropriate sedation for block performance and patient acceptance, however, clinicians may be reluctant to use them due to the medico-legal concerns engendered by admonitions against performing blocks in sedated or anesthetized patients. Future efforts must be directed toward developing more objective and exacting nerve localization and injection monitoring techniques to more reliably detect and prevent intraneural injection. The results of these efforts will inevitably be of far greater importance to the future of PNBs and their role in practice of modern anesthesiology than over-reaching, unsubstantiated opinions and statements (Hadzic A et al., 2007). 


\section{METHODS AND MEANS TO DECREASE THE RISK PF NEUROLOGIC COMPLICATIONS ASSOCIATED WITH NERVE BLOCKS}

The published data suggests that neurologic complications of PNBs are relatively rare. However, their the severity of consequences and lack of prevention strategies continue to present a source of significant concern for both clinicians and patients. The main inciting mechanism of neurologic injury with PNBs appears to be an intrafascicular or intraneural injection. It is fortunate that peripheral nerves possess an inherent natural protection; intraneural injections often do not result in intrafascicular needle placement and therefore, do not necessarily lead to nerve injury. It is commonly suggested that the use of short-beveled needles and avoidance of excessive sedation and general anesthesia should be employed to decrease the risk of nerve injury. However, these commonly voiced recommendations have been recently challenged. In addition, avoidance of adequate premedication may have a significant negative impact by decreasing the patient's acceptance and satisfaction with PNBs. The relatively low incidence rate of complications with PNBs, coupled with the lack of objective documentation and means to more precisely monitor administration of nerve blocks make retrospective analyses of cases of nerve injury largely speculative with regard to the actual mechanism of nerve injury in clinical practice (Hadzic A et al., 2007).

The following recommendations are suggested to decrease the risk of complications with PNBs:

\section{Aseptic technique}

Most nerve block techniques are merely percutaneous injections. However, infections are known to occur and can result in significant disability. Since this complication is almost entirely preventable, every effort should be made to adhere to strictly aseptic technique (Auroy Y et al., 2002).

\section{Short bevel insulated needles}

Insulated needles are now widely available and result in much more precise needle placement. The short bevel design helps prevent nerve penetration (Rice A and McMahon S, 1992).

\section{Needles of appropriate length for each block technique}

Excessively long needles should not be used for nerve blockade. For instance, never use needles longer than $50 \mathrm{~mm}$ for an interscalene block. In addition to the safety reasons, needles of appropriate length are also advanced with far greater precision than excessively long needles (Rice A and McMahon S, 1992).

\section{Surface localization}

In patients with difficult anatomy, surface localization of superficially seated nerves or plexuses can help reduce the number of needle passes (Auroy Y et al., 2002).

\section{Needle advancement}

During needle localization, advance and withdraw the needle slowly. Keep in mind that nerve stimulators deliver current of very short duration once $(1 \mathrm{~Hz})$ or twice $(2 \mathrm{~Hz})$ a second and that no current is delivered between the pulses. Thus, fast insertions and withdrawal of the needle passages may result in failure to stimulate the nerve because the needle may pass near by, or even through, the nerve between the stimuli without eliciting nerve stimulation (Rice A and McMahon S, 1992).

\section{Fractionated injections}

Inject smaller doses and volumes of local anesthetics (3-5 mL) with intermittent aspiration to avoid inadvertent intravascular injection. Always observe the patient during the injection of local anesthetic because negative aspiration of blood is not always present with an intravenous injection. This approach may allow detection of the signs of local anesthetic toxicity before the entire dose is injected (Auroy $\mathbf{Y}$ et al., 2002).

\section{Accuracy of the nerve stimulator}

Always make sure that the nerve stimulator is operational, delivering the specified current, and that the leads are properly connected to the patient and the needle (Choyce A et al., 2001).

\section{Avoidance of forceful, fast injections}

Forceful, fast injections are more likely to result in channeling of local anesthetic to the unwanted tissue layers, lymphatic vessels, or small veins that may have been cut during needle advancement. Such injections may result in massive channeling of the local anesthetic in the systemic circulation, with consequent risk of severe CNS and 
cardiac toxicity. Finally, forceful, fast injections under excessive pressure are more likely to result in an unrecognized intraneuronal injection. Limit the injection speed to $15-20 \mathrm{~mL} /$ minute (Auroy Y et al., 2002).

\title{
Avoidance of injection against abnormal resistance
}

Intraneuronal needle placement may result in high resistance (pressure) to injection due to the compact nature of the neuronal tissue and its connective tissue sheaths. Always use the same syringe and needle size to develop a "feel" during the injection. As a rule, when injection of the first $1 \mathrm{~mL}$ of local anesthetic proves difficult, the needle should be slightly withdrawn and the injection attempted again. If the resistance persists, the needle should be completely withdrawn and flushed before repeating the insertion. Ultimately, objective injection pressure monitoring will likely become a standard monitor to standardize nerve block injections, reduce the risk of intraneural injection and for medico-legal documentation (Selander D et al., 1978).

\begin{abstract}
Abort injection if pain is reported by the patient
Severe pain or discomfort on injection may signify intraneuronal placement of the needle and should be avoided. When this occurs, the injection should be abandoned, although the chances are that a damage may have already been caused at the time when the pain occurs. Lancinating, "shooting" pain on injection should not be confused with a mild "paresthesia-like" report by the patient when the needle is placed in the immediate vicinity to the nerve or plexus. In this case, local anesthetic can be injected slowly, provided that the resistance to injection is normal $(<20$ psi). Resuming the injection after waiting to see whether the pain will go away should not be done under any circumstances (Hadzic A et al., 2007).
\end{abstract}

\section{Chose your local anesthetic solution wisely}

Always choose a shorter acting (and less toxic) local anesthetic for short procedures where long-lasting postoperative analgesia is not required. Local anesthetic toxicity is the most common complication with neuronal blockade; The risk of sever toxicity is substantially lower with chloroprocaine or lidocaine than with bupivacaine.

\section{Blocks in anesthetized patients}

In the absence of reliable monitoring, blocks in anesthetized patients should still not be a common practice. When it is necessary to place blocks in anesthetized patients, this should be done by practitioners with experience and procons documented in the chart. The introduction of ultrasound-guided nerve blocks and injection pressure monitoring will likely change the practice and allow more routine performance of nerve blocks in anesthetized patients (Auroy Y et al., 2002).

\section{Repeating blocks after a failed block}

Repeating a block after a failed block should be avoided whenever possible. When indicated, it should be done only by those with substantial experience in the planned technique. Avoidance of abnormal resistance to injection or objective injection pressure monitoring is of utmost importance here as the clinician can no longer count on pain on injection as a sign of intraneural injection (Hadzic A et al., 2007).

\section{Management of patients with neurologic injury}

Peripheral nerve injury after peripheral nerve blocks: diagnosis, prognosis, and treatment Nerve injury is recognized as a potential complication of peripheral nerve block anesthesia, but fortunately, severe or disabling injuries are rare. When they do occur, they can be a frightening complication for the patient, the surgeon, and the anesthesiologist. Fortunately, most symptoms of nerve injury usually resolve in four to six weeks in over $95 \%$ of patients, and in $99 \%$ of the patients by one year. Early intervention may help prevent the long-term sequelae that can occur with unrecognized, and therefore improperly treated nerve injuries. With the resurgence of interest and utilization of peripheral nerve block anesthesia, it is important to develop a coinciding plan for management of postoperative nerve injuries. The plan should include recognition, diagnosis, and treatment of the nerve injury. A working knowledge of the long-term prognosis, understanding the importance of appropriate neurology consultations and familiarity with available diagnostic tests and treatment options is essential for the proper management of patients with neurologic injury.

With any peripheral nerve block, inclusive documentation of the block is of utmost importance for diagnostic, therapeutic and medicolegal purposes. Documentation that includes the nerve(s) stimulated, the minimum current used, the number of attempts, the appearance of pain or paresthesia during the procedure and measures taken, resistance to injection and injection pressure if pressure monitoring is used, type/dose of local anesthetic agent, and 
patient condition during the block is essential in understanding the mechanism of the injury. This documentation can help the anesthesiologist and/or consulting specialist determine the possibly etiology of the injury, any associated conditions, and guide treatment modalities (Urmey W and Stanton J, 2002).

\section{Mechanisms of Injury/Symptoms:}

Following peripheral nerve anesthesia, if sensory and/or motor function remains depressed beyond the expected duration of action of the local anesthetic, potential causes for the neurologic deficits should be investigated. Neurologic deficits may be related to vascular injuries, compression injuries, local anesthetic action, or traumatic nerve injury with fascicular disruption. When faced with a neurologic deficit, especially in a patient in whom a peripheral nerve block has been placed, it is important to remember that there are many causes of nerve injury unrelated to the performance of the regional anesthetic.

Other factors, i.e. tourniquet use, improper positioning, postoperative swelling, surgical trauma, and pre-existing neurologic deficits may be contributing or causative influences. Proper and objective documentation of the nerve block procedures can go a long way in deciphering whether the injury was caused by a nerve block, surgery or other factors.

\section{a. Vascular Injuries}

When symptoms occur early in the postoperative period (i.e. within minutes to hours), the anesthesiologist should suspect a vascular or compression-type injury due to disruption of the blood supply from an insult to the vascular structures, from hematoma at the surgical or block site, or from deep venous thrombosis. This is particularly true should a neurologic deficit appear after apparent resolution of the block (Ben-David B and Stahl S, 1999).

\section{b. Compression Injuries}

Compression neuropathies usually present as a focal mononeuropathy at sites where nerves pass through tissue tunnels, i.e. median through carpal tunnel, spinal nerve through vertebral foramina. Acute compression injuries can develop secondary to limb tourniquet paralysis, retractors used for surgical procedures, from expanding hematomas, or from improper intraoperative positioning, i.e. stretching of the cords of the brachial plexus during sternal retraction, or extending/pronating the forearm causing ulnar nerve compression. A compression injury can also occur with peripheral nerve block anesthesia from increased endoneural fluid pressure. This can occur with injection into a tight tissue compartment or when high injection pressures are used during nerve block administration. Metabolic diseases such as diabetes mellitus may make nerves more susceptible to compression injuries (Burns T, 2005).

\section{c. Local Anesthetic Action}

It is important to remember that neurologic deficits may be related to the residual neurologic blockade. Long-acting local anesthetics such as ropivacaine or bupivacaine have been reported to last up to twenty-four hours. It is nowadays well recognized that intraneural injections can and do occur without obligatory neurologic injury. Most such injections result in intraneural, but perifascicular injection of local anesthetic and their hallmark is a block of much longer duration than expected. When additives are included in the local anesthetic mixture, particularly in the setting of an intraneural injection, the block can be prolonged up to forty-eight hours. In the elderly and in those with pre-existing neurologic diseases, local anesthetic action can be prolonged secondary to abnormal uptake or reduced perfusion to the nervous tissue. The site of the block may have an effect on the duration of action of the local anesthetic. Therefore, in patients with known or occult preexisting neurologic diseases, the elderly, and in those treated with long-acting local anesthetics, sufficient time should be allowed for the local anesthetic to be thoroughly metabolized. Physical examination of these patients will usually reveal a slowly receding block. Once the return of motor function occurs, the block will usually recede in an accelerated fashion. If motor function returns with persistent sensory deficits, or motor dysfunction persists, further evaluation is necessary (Finucane B and Terblanche 0,2005$)$.

\section{d. Traumatic/Toxic Nerve Injury}

Nerve injury following peripheral nerve blocks may be caused by direct needle trauma, intraneural injection or local anesthetic neurotoxicity. Several animal studies have demonstrated that the frequency of nerve injury is greater with long beveled needles, but the duration and severity of the injury is greater with short beveled needles. When the bevel orientation is perpendicular instead of parallel to the nerve fibers, studies have shown that nerve injury can be more severe. Intraneural intrafascicular injection is characterized by high injection pressures at the onset of 
injection. Such injections are characterized by a prolong blockade followed by incomplete resolution and residual neurologic deficit. Because miniscule amount of local anesthetic (as little as $0.5 \mathrm{ml}$ ) is required to rupture the fascicle, intraneural injection should be suspected whenever there is resistance to injection or when the patient experiences severe pain on injection (Hadzic A et al., 2004).

All of the local anesthetics are potentially neurotoxic. Neurotoxicity is dependent on the dose, concentration, and length of exposure to the nerve tissue. Vasoconstrictive additives such as epinephrine that can affect neural blood flow may potentiate nerve injuries. Local anesthetic neurotoxicity is more prevalent in patients with pre-existing neurologic deficits, especially those with demyelinating diseases. In several studies of patients with occult or diagnosed multiple sclerosis, local anesthetic action was prolonged. The prolonged duration was thought to be related to an abnormal uptake of local anesthetic in demyelinated nerves (Finucane B and Terblanche $\mathbf{0 , 2 0 0 5 ) .}$

\section{Magnitude of Neural Injury:}

The degree of neural insult can be defined by the the terms neurapraxia, axonotmesis, and neurotmesis. The mildest form, neurapraxia, denotes a mild degree of neural insult with failure of impulse conduction across the affected segment. The electromyogram (EMG) is normal, but conduction velocity as demonstrated by a nerve conduction study (NCS) is decreased. Usually when the offending cause has been removed, recovery occurs over a variable amount of time that may be as long as several weeks. Complete recovery can usually be anticipated. Axonotmesis, an intermediate injury, occurs when there is axonal disruption with preservation of the endoneurium. Recovery is dependent on the rate of axonal regeneration (1-3 mm/day). If recovery occurs, it is most likely to be prolonged and incomplete. The prognosis is better for young, healthy patients with distal lesions. The most severe form of neural injury is neurotmesis; a complete transection or crushing of the nerve with disruption of the endoneurium. Surgical repair is usually indicated, but even then, recovery is usually incomplete. Prognosis for full recovery is poor (Lambert L et al., 1994).

\section{PATIENT EVALUATION/PHYSICAL EXAMINATION SYMPTOMS OF NERVE INJURY}

The symptoms of a nerve lesion after peripheral nerve block manifest after the block has receded; usually within 48 hours. The perception of symptoms is influenced by the origin of the nerve lesion and other confounding factors, such as postoperative pain, immobility, effects of surgery, position, application of casts, dressing, bandaging, and so forth. The intensity and duration of symptoms may also vary with the severity of the injury, from a light, intermittent tingling and numbness lasting a few weeks to a persistent, painful paresthesia, neuropathic pain, sensory loss, and/or motor weakness lasting for several months or years. Some nerve injuries may even evolve into a severe causalgia or reflex sympathetic dystrophy. It should be kept in mind that although dermatomes can provide clues to the location of injuries, the loss of sensation at the skin does not provide precise information concerning the site of injury because the boundaries of dermatomes are not precise, clearly defined lines. More useful information can be obtained from the loss of motor function on the basis of the origin and assessment of motor performance (Auroy $\mathbf{Y}$ et al., 1997).

The symptoms of neurological injury may range from mild to severe. Typical symptoms include dyesthesia, paresthesia, numbness, weakness, and pain. Symptoms of nerve injury may be acute or may not become clinically apparent for several days to several weeks. The approach to patient evaluation should be indistinguishable regardless of the timing of the suspected injury. Physical examination should include an assessment of vascular integrity, sensory/motor function, and reflexes.

\section{a. Vascular Mediated}

Vascular-mediated injuries include injury to arteries, veins, the formation of deep vein thrombosis, or a combination of any of these, creating vascular compromise.

Vascular injury with associated ischemia should be immediately. The examination should include an assessment of both venous and arterial circulation, skin color changes, the presence of engorgement or edema, hematoma or pain at the surgical or block site. If a vascular injury is suspected, the surgical team should be consulted immediately to manage and treat the problem. For example, patients with lower extremity joint replacement surgeries are prone to the development of deep venous thrombosis. Patients may be completely asymptomatic, or they may complain of limb pain, limb swelling, or leg pain on dorsiflexion of the foot. If the patient is receiving a continuous femoral nerve block, (typical after this type of surgery), and complains of pain behind the knee (sciatic distribution), the 
anesthesiologist must be cognizant of the possibility of a deep venous thrombosis vs incisional pain not covered by the femoral block. Occlusive dressing should be evaluated to make sure that they are not contributing to the vascular insult. With arterial compromise, the patient will complain of intense pain, paresthesia, and cold. The onset is usually rapid. A doppler/vascular study is the most common diagnostic test used to aid in the diagnosis. Arterial occlusion or compromise will usually require emergent surgical exploration (Auroy $Y$ et al., 1997).

\section{b. Neurologically Mediated}

If the physical examination demonstrates intact circulation, then a more thorough neurologic examination should be initiated. This includes a comprehensive motor and sensory examination as well as assessment of the reflexes. In multiple studies evaluating the incidence and prognosis of nerve injury after peripheral nerve block anesthesia, the majority of mild symptoms such as dyesthesia, mild paresthesia or numbness, or mild pain resolved within the first few days or weeks of the insult. Further diagnostic testing was not indicated (Auroy Y et al., 1997).

If the neurologic deficit is persistent or more severe (i.e. moderate to severe numbness, weakness and/or pain) then an evaluation by a neurologist may be indicated. For the hospitalized patient, the neurology consultation and subsequent examination should take place during the hospitalization. If the patient has already been discharged, a neurologist can be contacted for an outpatient evaluation. The magnitude or the rate of progression of symptoms will influence the urgency of the neurology consultation. Temporizing measures for pain control may be initiated until the patient can be evaluated. The neurology assessment will involve a more meticulous clinical examination to try to evaluate the degree of deficit and to determine what further diagnostic tests are indicated (Auroy Y et al., 1997).

\section{Diagnostic Tests}

There is a variety of diagnostic tests available for the detection of nerve injury. Each test has specific indications, advantages and disadvantages depending on the injury, the patient characteristics, the availability of the diagnostic equipment, and the expertise of the operator. The diagnostic tests currently available include electrophysiology, high-resolution ultrasound, and magnetic resonance imaging.

\section{a. Electrophysiology Testing}

Electrophysiology testing is important for defining the neurogenic basis of weakness, for localizing the site of the lesion, and to aid in determining the site of the lesion. Nerve conduction studies evaluate the sensory and motor signal amplitude and conduction velocity in myelinated nerves. In sensory nerves, changes in amplitude relate to the degree of axonal damage, while changes in conduction velocity relate to myelin damage. In motor nerves, changes in amplitude and conduction velocity are more difficult to localize because the signal involves the entire motor unit (i.e. the motor axon, neuromuscular junction, muscle fibers). If an isolated motor deficit is elicited, then the presence of disease involving the neuromuscular junction or muscle itself may need to be evaluated.

The indication for a nerve conduction study is weakness, numbness, or paresthesia. If nerve injury has occurred, changes in the nerve conduction study can be seen within days. If the complaint is strictly pain, a nerve conduction study is unlikely to detect any injury because only the signals transmitted via myelinated fibers are evaluated by this test; the function of unmyelinated axons. i.e. those responsible for pain transmission, is not evaluated. Therefore, the patient can have a normal nerve conduction study and still suffer from pain.

If the initial study is normal, there is a strong probability that the patient has neuropraxia which should resolve over time. Therefore, the patient should be followed clinically for improvement of symptoms. If symptoms persist or worsen, the study may be repeated in four to six weeks. If the initial study is abnormal, the contralateral limb should be evaluated to rule out systemic neurological disease such as diabetic neuropathy. If the study in the contralateral limb is normal, then electromyography may be considered (Hadzic A et al., 2007).

Electromyography involves recording of the electrical activity in muscle via a needle electrode inserted within the muscle. When an EMG needle is placed in a normal muscle at rest, there is no electrical activity. When nerve injury occurs, spontaneous muscle fasciculation appear in a resting muscle within two to three weeks of the insult. As muscle is released from control by the nerve and contractions begin to occur spontaneously, the specific changes seen on the electromyography are positive waves and fibrillations. A positive nerve conduction study detects the presence of a lesion, whereas the EMG can further localize the lesion to a specific site of injury on the nerve. For example, a patient develops weakness in the hand grip after an open reduction, internal fixation of the distal radius under infraclavicular brachial plexus block. A nerve conduction study demonstrates altered conduction in the 
distribution of the median nerve. An EMG may be able to localize the lesion to the surgical site or to the block site by evaluating groups of muscles innervated by the median nerve. If the intrinsic muscles of the hand are affected, but the intrinsic muscles of the forearm are unaffected, then the site of injury would appear to be at the wrist and not at the brachial plexus. Although some authors promote early EMG testing with repeat EMG's at intervals, in reality, most patients will not agree to repeat testing. The major disadvantage of EMG's are that they are painful procedures performed on awake patients. This test should be utilized when the highest probability of capturing signs of deinnervation are present, i.e. two to four weeks after the initial injury. If the test is delayed beyond that time interval, signs of re-innervation, i.e. long-duration, high-amplitude, polyphasic motor unit potentials, become present (Hadzic A et al., 2007).

\section{b. Doppler Ultrasound/High Frequency Ultrasound}

Doppler ultrasound provides both functional and structural information about the image being scanned. Most commonly it is used to study the carotid arteries and major arteries and veins of the lower extremities. It can be also be used to examine the arteries at the wrist, in the palms, and in the digits. It is the most common diagnostic test for deep venous thrombosis (Fornage B, 1988).

A newer type of ultrasound, high frequency ultrasound, is an effective imaging modality for identifying morphologic changes in the peripheral nerves. It is being used to evaluate nerve rupture, inflammatory processes, and compressive syndromes in peripheral nerves. It is an excellent test to localize the lesion, possibly providing an alternative to EMG. But because is does not test for the functional capacity of the nerve, it should probably be combined with a nerve conduction study. It may be a useful tool for initial imaging of a suspected peripheral nerve injury or for follow-up imaging. It is faster and more cost-effective than magnetic resonance imaging, and avoids the problems associated with claustrophobia. But it has limitations in that the test is operator dependent, requires an extended learning curve to obtain and interpret images, and is limited to nerves within $2 \mathrm{~cm}$ of the skin surface. At greater depths, coarser quality ultrasound nerve images can be obtained but they are difficult to distinguish from other tissues such as tendons (Silvestri E et al., 1995).

\section{c. Magnetic Resonance Imaging/Magnetic Resonance Neurography}

Magnetic resonance imaging, specifically magnetic resonance neurography, is a relatively new imaging technique that can reliably and selectively image peripheral nerves.135 By manipulating MRI parameters, nerves can be made to show up as three-dimensional "neurographic" images, comparable to angiograms. When it is properly implemented, MR neurography is capable of providing high-quality information about nerve compression, nerve inflammation, nerve trauma, systemic neuropathies, and recovery of nerves from pathologic states (Filler A et al., 1996).

Direct nerve imaging has been shown to demonstrate nerve continuity, distinguish intraneural from perineural masses, and localize nerve compressions prior to surgical exploration. Like ultrasound, its indication for acute peripheral nerve injury is in localizing the lesion. In some studies, MR signal alterations were shown to occur as early as twenty-four hours after an axonal nerve lesion, possibly making it the earliest form of detection of nerve injury. MR studies are limited by access to the patient, cost, longer scanning times and equipment availability (Filler A et al., 2004).

\section{Treatment Options For Peripheral Nerve Injury}

Once a nerve injury has occurred, treatment of the patient depends on the severity of the injury. It is prudent to remember that the majority of these injuries resolve with time. The care of the patient with a nerve injury can be divided into two groups, those with minor deficits and those with major deficits. Regardless of the type of injury, the patient should be evaluated clinically to ascertain that a more serious or occult injury or correctable contributing condition is not overlooked.

\section{Minor Deficits}

If the examination reveals minor deficits, reassurance is key to relieving the patient's anxiety. Further diagnostic testing or therapy is usually not indicated. Weekly to bi-weekly telephone follow-up to assess resolution of the injury by the pain management team will provide reassurance, allow early detection of more serious problems, and instill confidence in the patient. It is important to remember that one bad experience with regional anesthesia will often create a negative attitude toward future application for the patient, his family, and possibly the surgical team. 
Providing the patient with consistent, caring follow-up, despite the injury, will help to rebuild patient confidence in future anesthetic experiences.

\section{Major Deficits:}

If the clinical examination of the patient reveals a major neurologic deficit, early neurologic and/or neurosurgery consultation is advisable. In addition to a complete neurologic examination, the neurologist will determine the most appropriate diagnostic test for the patient. For example, if a compression injury is suspected, ultrasonography or MRI of the involved plexus may be performed. In patients with pain, medication for pain should be started early to prevent nerve sensitization. Physical and occupational therapy consults will be obtained by neurology with arrangements made for treatment after the patient is discharged. A consultation for social services may be necessary if the injury will impact the patient's ability to perform activities of daily living. Similarly, early consultation with physical therapy is a must to reduce the risk of contractures, muscle atrophy and prolonged disability. Close followup by neurology should be arranged, and well as continued follow-up by anesthesiology until the injury is completely resolved or is stable.

\section{Drug Therapy}

Multiple drugs are effective to treat neurally-mediated pain. The more commonly prescribed medications include tricyclic antidepressants, serotonin reuptake inhibitors, anticonvulsants, opiods, and capsaicin cream. These drugs should be administered under the direction of a chronic pain specialist or a neurologist who has evaluated the patient and has the capacity to do follow-up evaluations.

a. Tricylic Antidepressants

Amitriptyline is among the more efficacious drugs. It has multiple effects including blocking the reuptake of serotonin and norepinephrine, blocking the NMDA receptor, and blocking voltage dependent sodium channels. A more recent study demonstrated that amitriptyline has an ability to maintain spinal cord GABA(B) receptor activity. The drug can be started at $10 \mathrm{mg}$ to $25 \mathrm{mg}$ daily, and escalated by $25 \mathrm{mg}$ every week to an effected level. Doses should be limited to $100 \mathrm{mg}$ daily. Side effects include decreased salivation, constipation, urinary hesitancy, blurred vision, orthostatic hypotension, sedation and cognitive impairment. One very serious side effect in cardiac patients is torsade-de-point from prolongation of the Q-T interval, but this has been shown to occur only in higher doses (Ke M et al., 2005).

b. Selective Serotonin Reuptake Inhibitors:

Paroxetine is the only selective serotonin reuptake inhibitor that might be effective in the treatment of neurallyderived pain. In a study comparing paroxetine to imipramine (another TCA), paroxetine was as effective in 60-70\% of the patients receiving the drug. Because of its marginal effectiveness, it is reserved only for those patients unable to tolerate other classes of medications (Sindrup S et al., 1992).

\section{c. Anticonvulsants}

Commonly prescribed anticonvulsants in the treatment of minor neural changes, i.e. pain and paresthesia, are gabapentin and carbamazepine. Gabapentin is an amino acid derivative of GABA (gamma-amino butyric acid). The proposed mechanism of analgesic action is related to its effect on sensitized neurons. It is thought to directly or indirectly inhibit excitatory neurotransmitters, block neuronal calcium channels, and augment central nervous system inhibitory pathways by increasing GABA transmission. Gabapentin is usually started at $300 \mathrm{mg}$ daily with increasing doses to 1300 to $1800 \mathrm{mg}$ per day in divided doses. Side effects of gabapentin include drowsiness, anxiety, visual disturbances, hypertension, and ataxia. Other anticonvulsants that have been used in the treatment of neurally-mediated pain are carbamazepine, topiramate, levetiracetam, and oxcarbazepine (Rogawski M and Loscher W, 2004).

\section{Opioids}

Opioid therapy is controversial, but may to be effective in alleviating pain of peripheral nerve injury origin. In five randomized, controlled trials of opioid therapy used for neuropathic pain, all demonstrated benefits of opioids in controlling pain. Commonly prescribed opiods for short-term therapy include oxycodone, oxycontin, or fentanyl patches. To prevent opioid dependence or tolerance, other therapeutic agents, i.e. amitriptyline, gabapentin, should be titrated up while titrating off the opioids (Watson C and Babul N, 1998). 


\section{Tramadol}

Tramadol is approved for use in the United States for moderate to severe pain. It binds to mu opioid receptors contributing to its analgesic properties. It inhibits the reuptake of serotonin and norepinephrine the the central nervous system. In recent literature, tramadol has been shown to be effective in the treatment of arthrosic and neuropathic pain, and in mixed nociceptive-neuropathic pain. It is tolerated by elderly patients, has a low incidence of constipation, is devoid of immunosuppressive activity, and has a low tendency toward tolerance. It has a minimum risk of addiction and abuse (Rowbotham M, 2005).

\section{Capsacion Ointment}

Capsaicin is a vanniloid compound most commonly found in hot peppers. Many of the neuropathies with pain as the predominant symptom involve small afferent fibers resistant to the analgesic action of the more commonly prescribed medications. Capsaicin can deplete the transmitter contents of these small afferent fibers leading to their degeneration and subsequent pain-generating ability. Its application is primarily for cutaneous hyperalgesia states such as postherpetic neuralgia. It is probably not effective for most painful neuropathies of deeper origin (Rowbotham M, 2005).

After starting medication therapy, consultant follow-up should be scheduled for two weeks after the injury to determine if the deficit has resolved, persisted, or worsened. If the deficit has resolved, the medication will most likely be discontinued. The patient should be instructed to notify the consultant if any neurologic changes develop within the next month. If the deficit persists, the dose of medication will be adjusted as described. During follow-up visits, the patient should be asked about any side effects associated with the prescribed medication. Subsequent follow-up depends on the nature of the deficit. If it is stable but persistant, consultant follow-up should be arranged for three to six months. If it has worsened, adjustments will be made in the drug therapy to try to alleviate symptoms. Follow-up will be determined by the consultant based on the patient's condition and symptoms (Hadzic A et al., 2007).

Despite meticulous care, patient selection, and experience, neurologic deficits after regional anesthesia may occur. The debate over performing blocks in awake or sedated/asleep patients is inconsequential since nerve injuries have occurred in both groups. Understanding the mechanism of injury, the typical clinical course following injury, the prognosis, and obtaining appropriate consults is essential knowledge for the regional anesthesiologist. A basic understanding of diagnostic tests and treatment modalities will provide the clinician with information necessary to participate in meaningful discussions with the patient and other specialists involved in the patient's care. Regardless of the consults obtained, the importance of follow-up by anesthesiology cannot be over-emphasized. Complete documentation of the initial consult, the block procedure, the post-operative care and follow-up will be extremely important in the event of any legal action as well as in the professional care of the patient (Hadzic A et al., 2007).

In closing, few publications have had a greater impact on the clinical practice of anesthesiology then the American Society of Anesthesiologists (ASA) practice guidelines.These practice guidelines have been designed to enhance and promote the safety of anesthetic practice and have made the practice of general anesthesia much safer. Such guidelines are much needed but currently do not exist with regards to the practice of PNBs. This is most likely because administration of PNBs has been traditionally based on individual preferences, clinical impressions and other subjective methods. Future efforts should be directed toward developing more objective and exacting nerve localization and injection monitoring techniques to more reliably detect and prevent intraneural intrafascicular injection. The results of these efforts will inevitably be of crucial importance to the future of PNBs and their role in practice of modern anesthesiology (Rowbotham M, 2005).

\section{References:-}

1. Adam F, Jaziri S, Chauvin M: Psoas abscess complicating femoral nerve block catheter. Anesthesiology 2003; 99: 230-231

2. Albin MS: Air Embolism. Anesthesiology Clinics of North America 11(1) :1-24,1993

3. Al-Nasser B, Palacios J: Femoral nerve injury complicating continuous psoas compartment block. Reg Anesth Pain Med 2004; 44: 361-63

4. Applebaum RM, Adelman MA, Kanschuger MS, Jacobowitz G, Kronzon I : Transesophageal echocardiographic identification of a retrograde dissection of the ascending aorta caused by inadvertent cannulation of the common carotid artery. J Am Soc Echocardiogr 1997 Sep;10(7):749-51 
5. Arnold HL, OdomRB, JamesWD: Andrew's Diseases of the skin-Clinical dermatology, 8th edition, An HBJ International Edition 1 Children with Tracheostomies Resource Guide, by Marilyn K. Kertoy, page 15,1990

6. Atlee AL: Complications in Anesthesia. W.B. Saunders, 1999.

7. Auroy Y, Narchi P, Messiah A, Litt L, Rouvier B , Sami K : Serious complications related to regional anesthesia : Results of a prospective survey in France . Anesthesiology 1997 ; $87: 479-86$

8. Auroy Y, Benhamou D, Bargues L: Major complications of regional anesthesia in France: The SOS regional anesthesia hotline service. 2002; Anesthesiology: 1274-80.

9. Barash, Cullen \& Stoelting: (Clinical Anesthesia) 5th Edition. Copyright 2001, Lippincott, Williams \& Wilkins, Philadelphia, PA. Ch. 24, pp 639-665

10. Barutell C, Vidal F, Raich M, Montero A: A neurological complication following interscalene brachial plexus block. Anaesthesia 1980; 35: 365-7

11. Bashein G, Robertson H, Kenndey W: Persistent phrenic nerve paresis following interscalene brachial plexus block. Anesthesiology 1985; 63: 102-104

12. Ben-David B, Stahl S: Axillary block complicatged by hematoma and radial nerve injury. Regional Anesthesia and Pain Medicine 1999; 24: 264-266

13. Bendszus M, Wessig C, Solymosi L, Reiners K, Koltzenburg M: MRI of peripheral nerve degeneration and regeneration: correlation with electrophysiology and histology. Experimental Neurology 2004; 188: 171 177

14. Bentley F, Schlapp W: The effects of pressure on the conduction in peripheral nerves. Journal of Physiology 1943; 102: 72

15. Benumof J: Permanent loss of cervical spinal cord function associated with interscalene block performed under general anesthesia. Anesthesiology 2000; 93: 1541-1544

16. Bernards C, Carpenter R, Rupp S, Brown DL, Morse B, Morell R, Thompson G: Effect of midazolam and diazepam premedication on central nervous system and cardiovascular toxicity of bupivacaine in pigs. Anesthesiology 1989; 70: 318-323

17. Bhananker S, Domino K: What actions can be used to prevent peripheral nerve injury. In Evidence-Based Practice of Anesthesiology. Edited by LA F. Philadelphia, Elsevier Inc, 2004, pp 228-235

18. Biboulet $\mathbf{P}$, Aubas $\mathbf{P}$, Dubourdieu $\mathbf{J}$, Rubenovitch $\mathbf{J}$, Capdevila X , d'Athis $\mathbf{F}$ : Fatal and non-fatal cardiac arrests related to anesthesia . Can J Anesth $2001 ; 48: 326-32$

19. Bogdanov A, Loveland R: Is there a place for interscalane block performed after induction of general anesthesia? Eur J Anesthesiol 2005; 22: 107-110

20. Bonner S, Pridie A: Sciatic nerve palsy following uneventful sciatic nerve block. Anaesthesia 1997; 52: 1205-1211

21. Borgeat A, Ekatodramis G, Kalberer F: Acute and non-acute complications associated with interscalene block and shoulder surgery. A prospective study. Anesthesiology 2001; 95: 875-880

22. Borgeat A, Dullenkopf A, Ekatodramis G, Nagy L: Evaluation of the lateral modified approach for continuous interscalene block after shoulder surgery. Anesthesiology 2003; 99: 436-42

23. Boyle DM, Engelking C: Vesicant extravasation: myths and realities. Oncol Nurs Forum 1995;22:57-67. Brown D: Local anesthetics and regional anesthesia equipment, Atlas of regional anesthesia. Edited by DL B. Philadelphia, W.B. Saunders Company, 1992, pp 3-11

24. Breslin D, Martin G, Macleod D, D'Ecole F, Grant S: Central nerveous system toxicity folloing the administration of levobupivacaine for lumbar plexus block: A report of two cases. Reg Anesth Pain Med 2003; 28: 144-7

25. Bromage P: Masked Mischief. Reg Anesth Pain Med 1996; 21: 62-3

26. Brown A, Parker G: The use of a "reverse" axis (axillary-interscalene) block in a patient presenting with fractures of the left shoulder and elbow. Anesth Analg 2001; 93: 1618-20

27. Brown RH, Schauble JF, Miller NR: Anemia and hypotension as contributors to perioperative loss of vision. Anesthesiology 1994;80:222.

28. Burns S; Herbison GJ : Spinal accessory nerve injury as a complication of internal jugular vein cannulation. Ann Intern Med, 125:700, 1996

29. Burns T: Mechanisms of acute and chronic compression neuropathy, Peripheral Neuropathy, 4th Edition. Edited by Dyck P, Thomas P. Philadephia, Elsivier-Saunders, 2005, pp 1391-1402

30. Candido K, Sukhani R, Doty R, Nader A, Kendall M, Yaghnour E, Kataria T, McCarthy R: Neurologic sequelae after interscalene brachial plexus block for shoulder/upper arm surgery: the association of patient, anesthetic, and surgical factors to the incidence and clinical course. Anesthe Analg 2005; 100: 1489-95 
31. Capdevila X, Macaire P, Dadure C, Choquet, Biboulet P, Ryckwaert Y, D'Athis F: Continuous psoas compartment block for postoperative analgesia after total hip arthroplasty: New landmarks, technical guidelines and clinical evaluation. Anesth Analg 2002; 94: 1606-1613.

32. Caplan R , Posner K , Cheney F : Effect of outcome on physician judgments of appropriateness of care . JAMA $1991 ; 265: 1957-60$

33. Caplan RA: Will we ever understand perioperative neuropathy? A fresh approach offers hope and insight (editorial). A nesthesiology 1999; 91: 335-6

34. Casati A, Fanelli G, Beccaria P: The effects of the single or multiple injection technique on the onset time of femoral nerve blocks with $0.75 \%$ ropivacaine. Anesth Analg 2000; 91: 181-4

35. Casati A, Fanelli G, Beccaria P: The effects of single or multiple injections on the volume of $0.5 \%$ ropivacaine required for femoral nerve blockade. Anesth Analg 2001; 93: 183-6

36. Chazotte B, Vanderkooi G: Multiple sites of inhibition of mitochondrial electron transport by local anesthetics. Biochemisty Biophysics Acta 1981; 636: 153-161

37. Chan OB and Michael JP : Persistent Cerebrospinal Fluid Leak: A Complication of the Combined SpinalEpidural Technique. Anesth Analg 2004;98:828-83

38. Chelly J: Nerve stimulator, Peripheral nerve blocks. A color atlas. Edited by Chelly J. Philadelpia, PA, Lippincot Williams \& Wilkins, 1999, pp 7-10

39. Cheney FW, Posner KL, Caplan RA, Gild WM: Burns from warming devices in anesthesia. Anesthesiology 1994;80:806.

40. Cheney FW, Domino KB, Caplan RA, Posner KL: Nerve injury associated with anesthesia: A closed claims analysis. Anesthesiology 1999; 90: 1062-9

41. Choyce A, Chan V, Middleton W, Knight P, Peng P, McCartney C: What is the relationship between paresthesia and nerve stimulation for axillary brachial plexus block? Reg Anesth Pain Med 2001; 26: 100-4

42. Christopherson R, Beattie C , Frank S, Norris E, Meinert C, Gottlieb S, Yates H, Rock P, Parker S , Perler B : Perioperative morbidity in patients randomized to epidural or general anesthesia for lower extremity vascular surgery. Perioperative Ischemia Randomized AnesthesiaTrial Study Group . Anesthesiology $1993 ; 79: 422-34$

43. Claudio RE, Hadzic A, Shih H, Vloka JD, Castro J, Koscielniak-Nielsen Z, Thys DM, Santos AC: Injection pressures by anesthesiologists during simulated peripheral nerve block. Reg Anesth Pain Med 2004; 29: 201-5

44. Contreras MG, Warner MA, Charboneau WJ: The anatomy of the ulnar nerve at the elbow: Potential relationship of acute ulnar neuropathy to gender differences. Clin Anat 11:372-378, 1998.

45. Contreras MG, Warner MA, Carmicheal SW: Perioperative anterior interosseous neuropathy. Anesthesiology 96:243-245, 2002.

46. Coppieters MW, Van De Velde M, Stappaerts KH: Positioning in anesthesiology. Toward a better understanding of stretch-induced perioperative neuropathies. Anesthesiology 2002;97:75.

47. Cucchiara RF, Black S: Corneal abrasion during anesthesia surgery. Anesthesiology 69:978-979, 1988 .

48. Dahlgren N, Tornebrandt K: Neurologic complications after anaesthesia. A follow-up of 18000 spinal and epidural anaesthetics performed over three years. Acta Anaesthesiol Scand 1995; 39: 872-80.

49. David JS, Thomas JG: Airway management, Anesthesia, Miller RD (Editor), 3rd edition 1990;1287

50. Dawson D, Hallet M, Wilbourn A: Entrapment Neuropathies, 3rd Edition. Philadelphia, LippincottRaven, 1999

51. Drasner K, Sakura S, Chan VW, Bollen AW, Ciriales R: Persistent sacral sensory deficit induced by intrathecal local anesthetic infusion in the rat. Anesthesiology 1994; 80: 847-52

52. Du Pen SL, Peterson DG, Williams A, Bogosian A: Infection during chronic epidural catheterization: diagnosis and treatment. Anesthesiology 1990; 73: 905-9

53. Dutton RP, Eckhardt WF, Sunder N: Total spinal anesthesia after interscalene blockade of the brachial plexus. Anesthesiology 1994; 80: 939-41

54. Dylewsky W, McAlpine FS: Peripheral nervous system. In Martin JT, Warner MA (eds): Positioning in Anesthesia and Surgery, 3rd ed. Philadelphia, WB Saunders, 1997.

55. Eckhardt WF, Iaconetti DJ, Kwon JS: Inadvertent Carotid Artery Cannulation During Pulmonary Artery Catheter Insertion. J Cardiothorac Vasc Anesth 1996, 283-290

56. Edde R, Deutsch S: Cardiac arrest after interscalene brachial plexus block. Anesth Analg 1977; 56: 446-7

57. Ediale K, Myung C, Neuman G: Prolonged Hemidiaphragmatic paralysis following interscalene brachial plexus block. J Clin Anesth 2004; 16: 573-575 
58. Fagiolini M, Caleo M, Strettoi E, Maffei L: Axonal gtransport blockade in the neonatal rat optic nerve induces limited retinal ganglion cell death. Journal of Neuroscience 1997; 17: 7045-7052.

59. Fanelli G, Casati A, Garancini P, Torri G: Nerve stimulator and multiple injection technique for upper and lower limb blockade: Failure rate, patient acceptance, and neurologic complications. Anesth Analg 1999; 88: $847-52$

60. Fanelli G, Casati A, Beccaria P: Interscalene brachial plexus anaesthesia with small volumes of ropivacaine $0.75 \%$ : effects of the injection technique on the onset time of nerve blockade. Eur J Anaesthesiol 2001; 18: 54-8

61. Filler A, Hayes C, Kliot M, Winn H, Bell B, Tsuruda J: Magnetic resonance neurography. Lancet 1993; 341: 659-661

62. Filler A, Kliot M, Howe F, Hayes C, Saunders D, Goodkin R, Bell B, Winn H, Griffiths J, Tsuruda J: Application of magnetic resonance neurography in the evaluation of patients with peripheral nerve pathology. Journal of Neurosurgery 1996; 85: 299-309

63. Filler A, Maravilla K, Tsuruda J: MR neurography and muscle MR imaging for image diagnosis of disorders affecting the peripheral nerves and musculature. Neurology Clinics 2004; 22: 643-682

64. Findlay JY: White veins after propofol. Anaesthesia 1994;49:838.

65. Finucane B \& Terblanche O: Prolonged duration of anesthesia in a patient with multiple sclerosis following paravertebral block, Canadian Journal of Anesthesia, 2005, pp 454-8

66. Fornage B: Peripheral nerves of the extremities: imaging with US. Radiology 1988; 167: 179-182

67. Fortunato, Nancymarie: (Berry \& Kohn's, "Operating Room Technique") 9th Edition, Copyright 2000, Mosby,Inc. St. Louis, Missouri. Ch. 28, pp 487-507

68. Fournier R, Weber A, Gamulin Z: Posterior labat vs. olateral popliteal sciatic block: posterior sciatic block has quicker onset and shorter duration of anaesthesia. Acta Anaesthesiology Scandinavia 2005; 49: 683-6

69. Freedman JM, Li D, Drasner K, Jaskela MC, Larsen B, Wi S: Transient neurologic symptoms after spinal anesthesia. An epidemiologic study of 1863 patients. Anesthesiology 1998; 89: 633-41

70. Fremling M, Mackinnon S: Injection injury to the median nerve. Ann Plast Surg 1996; 37: 561-7

71. Gaertner E, Estebe J, Zamfir A, Cuby C, Macaire P: Infraclavicular plexus block: multiple injection versus single injection. Reg Anesth Pain Med 2002; 27: 590-4

72. Gault DT: Extravasation injuries. Br J Plast Surg 1993;46:91-6.

73. Gentili F, Hudson A, Hunter D: Clinical and experimental aspects of injection injuries of peripheral nerves. Can J Neurol Sci 1980; 7: 143-151

74. Gentili F, Hudson A, Kline D, Hunter D: Early changes following injection injury of peripheral nerves. Can J Surg 1980; 23: 177-82

75. Gerancher J, Viscusi E, Liguori G, McCartney C, Williams B, Ilfeld B, Grant S, Hebl J, Hadzic A: Development of a standardized peripheral nerve block procedure note form. Reg Anesth Pain Med 2005; 30: 67-71

76. Giaufre E, Dalens B, Gombert A: Epidemiology and morbidity of regional anesthesia in children: a oneyear prospective survey of the French-Language Society of Pediatric Anesthesiologists. Anesth Analg 1996; 83: 904-912

77. Gillespie J, Menk E, Middaugh R: Reflex sympathetic dystrophy. A complication of interscalene block. Anesth Analg 1987; 66: 1316-7

78. Goldberg M, Gregg C, Larijani G, Norris M, Marr A, Seltzer J: A comparison of three methods of axillary approach to brachial plexus blockade for upper extremeity. Anesthesiology 1987; 66: 814-6

79. Golden LR: Incidence and Management of Large-Bore Introducer Sheath Puncture of the Carotid Artery. J Cardiothorac Vasc Anesth 1995, 425-428

80. Hadzic A, Vloka J, Kuroda M : The practice of peripheral nerve blocks in the United States: a national survey. Reg Anesth Pain Med 1988; 23: 241-6

81. Hadzic A, Dilberovic F, Shah S : Combination of Intraneural Injection and High Injection Pressure Leads to Severe Fascicular Injury and Neurologic Deficits in Dogs. Reg Anesth Pain Med 2004; 29: 417-23

82. Hastings-Tolsma M, Yucha CB: IV infiltration: no clear signs, no clear treatment? RN 1994;57:34-8

83. Hodgson P, Neal J, Pollock J, Liu S: The neurotoxicity of drugs given intrathecally (spinal). Anesthesia and Analgesia 1999: 797-809

84. Hofmann H.S. , G. Rettig, J. Radke, H. Neef, R.E. Silber: Iatrogenic ruptures of the tracheobronchial tissue. Eur J Cardiothorac Surg 2002;21:649-652

85. Horlocker TT, McGregor DG, Matsushige DK, Schroeder DR, Besse JA: A retrospective review of 4767 consecutive spinal anesthetics: central nervous system complications. Anesth Analg 1997; 84: 578-84 
86. Howard M, Florence J, Roger S, Michael E: Nonoperative Management of Tracheal Laceration During Endotracheal Intubation Ann Thorac Surg 1997;63:240-242

87. Hudson A, Kline D, Gentili F: Management of peripheral nerve problems, Peripheral nerve injection injury. Edited by Omer G, Spinner M. Philadelphia, WB Saunders, 1980, pp 639-653

88. Hutschala D, Mascher H, Schmetterer L, Klimscha W, Fleck T, Eichler H, Tschernko E: Clonidine added to bupivacaine enhances and prolongs analgesia after brachial plexus block via a locl mechanism in healthy volunteers. European Journal of Anesthesia 2004; 21: 198-204

89. Irita K, Noda E, Karashima Y, Okamoto H, Takahashi S: Tension hemothorax caused by inadvertent insertion of an introducer dilator into the vertebral artery. J Cardiothorac Vasc Anesth. 1999 Apr;13(2):241-2.

90. Jankovic D, Wells C: Brachial plexus, Regional nerve blocks, 2nd Edition. Edited by Jankovic D, Wells C. Vienna, Blackwell Science Berlin, 2001, pp 58-86

91. Jankowski C, Keegan M, Bolton C, Harrison B: Neuropathy following axillary brachial plexus blocks: Is it the tourniquet? Anesthesiology 2003; 99: 1230-1232.

92. Jankowski C, Stuart M, Hebl J, Rock M, Pagnano M, Beighley C, Schroeder D, Horlocker T: A comparison of psoas compartment block and spinal and general anesthesia for outpatient knee arthroscopy. Anesth Analg 2003; 97: 1003-9

93. Johnson M, Saenz J, DaSilva A, Uhl C, Gores G: Effect of local anesthetic on neuronal cytoplasmic calcium and plasma membrane lysis (necrosis) in a cell culture model. Anesthesiology 2002; 96: 1466-1476

94. Johnson M, Uhl C, Spittler K, Wang H, Gores G: Mitochondrial injury and caspase activation by the local anesthetic lidocaine. Anesthesiology 2004; 101: 1184-1194

95. Kalichman MW, Calcutt NA: Local anesthetic-induced conduction block and nerve fiber injury in streptozotocin-diabetic rats. Anesthesiology 1992; 77: 941-7

96. Kanai Y, Katsuki H, Takasaki M: Graded irreversible changes in crayfish giant axon as manifestations of lidocaine neurotoxicity in vitro. Anesthesia and Analgesia 1998; 86: 569-573

97. Kaneko S, Matsumoto M, Tsuruta S, Hirata T, Gondo T, Sakabe T: The nerve root entry zone is highly vulnerable to intrathecal tetracaine in rabbits. Anesth Analg 2005; 101: 107-14.

98. Kassner E: Evaluation and treatment of chemotherapy extravasation injuries. J Pediatr Oncol Nurs 2000;17:135-48.

99. Katz J: Median nerve: Block at the elbow, Atlas of regional anesthesia, 2nd Edition. Edited by Katz J. East Norwalk, CT, Appleton \& Lange, 1994, pp 82-3

100. Kaufman B, Nystrom E, Nath S, Foucher G, Nystrom A: Debilitating chronic pain syndromes after presumed intraneural injections. Pain 2000; 85: 283-286

101. Kent K, Moscucci M, Mansour K, DiMattia S, Gallagher S, Kuntz R, Skillman J: Retroperitoneal hematoma after cardiac catheterization : Prevalence, risk factors, and optimal management. J Vasc Surg 1994; 20: 905-910.

102. Ke M, Ralya A, Reisman S, Enna S: Amitriptyline prevents thermal hyperalgesia and modifications in rat spinal cord GABA (B) receptor expression and function in an animal model of neuropathic pain. Biochemical Pharmacology 2005; 123-28

103. Kim J , Bahk J , Sung J : Influence of age and sex on the position of the conus medullaris and Tuffier's line in adults . Anesthesiology $2003 ; 99: 1359-63$

104. Kingsley R: The gross structure of the nervous system, Concise text of neuroscience. Edited by Kingsley R. Philadelphia, Lippincott, Williams, and Wilkins, 2000, pp 1-15

105. Kitagawa N, Oda M, Totoki T: Possible mechanism of irreversible nerve injury caused by local anesthetics: detergent properties of local anesthetics and membrane disruption. Anesthesiology 2004; 100: 962-7

106. Klein S, D'Ercole F, Greengrass R, Warner D: Enoxaparin associated with psoas hematoma and lumbar plexopathy after lumbar plexus block. Anesthesiology 1997; 87: 1576-1579

107. Koscielniak-Nielsen Z, Stens-Pedersen H, Lippert F: Readiness for surgery after axillary block: single or multiple injection techniques. Eur J Anaesth 1997; 14: 164-71

108. Koscielniak-Nielsen Z, Rotboll Nielsen P, Sorensen T, Stenor M: Low dose axillary block by targeted injections of the terminal nerves. Can J Anaesth 1999; 46: 658-664

109. Koscielniak-Nielsen Z, Rasmussen H, Nielsen P: Patients' perception of pain during axillary and humeral blocks using multiple nerve stimulations. Reg Anesth Pain Med 2004; 29: 328-32

110. Kua JS; Tan IK: Airway obstruction following internal jugular vein cannulation. Anaesthesia, 52:776-80, 1997 
111. Lambert L, Lambert D, Strichartz G: Irreversible conduction block in isolated nerve by high concentrations of local anesthetics. Anesthesiology 1994; 1994: 1082-1093

112. Lavoie J, Martin R, Tetrault J : Axillary plexus block using peripheral nerve stimulator: single or multiple injections. Can J Anaesth 1992; 39: 583-6

113. Lee LA, Roth S, Posner KL, Cheney FW, Caplan RA, Newman NJ, Domino KB: The American Society of Anesthesiologists Postoperative Visual Loss Registry: Analysis of 93 spine surgery cases with postoperative visual loss. ANESTHESIOLOGY 2006; 105:652-9

114. Lefor T : Barotrauma from Anesthesia Ventilators. Health Devices Hazard, Nov 1998;17(11):354-5

115. Lichtenfeld N: The pneumatic ankle tourniquet with ankle block anesthesia for foot surgery. Foot Ankle 1992; 13: 344-349

116. Lim E, Pereira R: Brachial plexus injury following brachial plexus block. Anesthesia and Analgesia 1984; 39: 691-4

117. Liu SS, Mulroy MF: Neuraxial anesthesia and analgesia in the presence of standard heparin. Reg Anesth Pain Med 1998; 23: 157-63

118. Lockhart PB, Feldbau EV, Gabel RS, Connolly SF, Silversin JB: Dental complications during and after tracheal intubation. J Am Dent Assoc 1996; 112: 480-3

119. Lundborg G: Nerve injury and repair. New York, Churchill Livingstone, 1988

120. Lynch N, Cofield R, Silbert P, Hermann R: Neurologic complications after total shoulder arthroplasty. Journal of Shoulder and Elbow Surgery 1996; 5: 53-61

121. Mackinnon S, Hudson A, Gentili F, Kline D, Hunter R: Peripheral nerve injury with steroid agents. Plast Reconstr Surg 1982; 69: 482-9

122. Mackinnon S, Hudson A, Llamas F, Dellon A, Kline D, Dan A, Hunter R: Peripheral nerve injury by chymopapain injection. J Neurosurg 1984; 61: 1-8

123. Mackinnon S, Dellon A: Classification of nerve injuries as the basis of treatment, Surgery of the peripheral nerve. Edited by Mackinnon SE DA. New York, Thieme Medical Publishers, Inc, 1988, pp 35-63

124. Mahli A, Coskun D, Akcali D: Aetiology of convulsions due to stellate ganglion block: a review and report of two cases. Eur J Anaesthesiol 2002; 19: 376-80

125. Majeski J: Vertebral arteriovenous fistula as a result of Swan-Ganz catheter insertion: surgical correction in a symptomatic patient. Int Surg 1999 Jan-Mar;84(1):74-7

126. Meier G, Bauereis C, Heinrich C: Interscalene brachial plexus catheter for anesthesia and postoperative pain therapy. Experience with a modified technique. Anaesthesist 1997; 46: 715-9

127. Mennim P; Coyle CF; Taylor JD: Venous air embolism associated with removal of central venous catheter. BMJ, 305:171-2, 1992 Jul 18

128. Middleton R, Varian J: Tourniquet paralysis. Aust N Z J Surg 1974; 44: 124-127

129. Miller JA, Singireddy S, Maldjian P, Baker SR: A reevaluation of the radiographically detectable complications of percutaneous venous access lines inserted by four subcutaneous approaches. Am Surg 1999 Feb;65(2):125-30

130. Millesi H, Terzis JK: Nomenclature in peripheral nerve surgery. Clin Palast Surg 1984; 11: 3-8

131. Morgan GE, Mikhali MS, Murray JM : Clinical Anaesthesiology, 5th edition McGraw-Hill, 2006; 77-79.

132. Mulroy F: Brachial plexus blocks, Regional anesthesia: An illustrated procedureal guide, 3rd Edition. Edited by Mulory F. Philadelphia, PA, Lippincott Williams \& Wilkins, 2002, pp pp157-82

133. Myers R, Heckman H: Effects of local anesthesia on nerve blood flow: studies using lidocaine with and without epinephrine. Anesthesiology 1989: 757-62

134. Napiontek M, Ruszkowski K: Paralytic drop foot and gluteal fibrosis after intramuscular injections. J Bone Joint Surg Br 1993; 75: 83-85

135. Nuttall GA, Garrity JA, Dearani JA, Abel MD, Schroeder DR, Mullany CJ: Risk factors for ischemic optic neuropathy after cardiopulmonary bypass: A matched case/control study. Anesth Analg 2001; 93:14106

136. Obach J, Aragones J, Ruano D: The infrapiriformis foramen syndrome resulting from intragluteal injection. J Neurol Sci 1983; 58: 135-142

137. Oda M, Kitagawa N, Sakurada T, Miyabe T, Totoki T, Morimoto M: Is the neurotoxic concentration predicted from solution property of local anesthetics agreement with that obtained from animal study. Anesthesiology 1998; 89: A1417

138. Ohtake K: Glutamate release and neuronal injury after intrathecal injection of local anesthetics. Neuroreport 2000; 11: 1105-1109 
139. Oropello JM, Leibowitz AB, Manasia A: Dilator-associated complication of central vein catheter insertion: possible mechanism of injury and suggestion for prevention. J Cardiothorac Vasc Anesth 1996; 10: 634-7

140. Ould-Ahmed M, Drouillard I, Fouirel D, Rouisally P, Almanza L, Segalen F: Convulsions induced by ropivacaine after midhumeral block. Ann Fr Anesth Reanim 2002; 21: 681-4

141. Paqueron X, Bouaziz H, Macalou D: The lateral approach to the sciatic nerve at the popliteal fossa: one or twoinjections? Anesth Analg 1999; 89: 1221-5

142. Passannante AN: Spinal anesthesia and permanent neurologic deficit after interscalene block. Anesth Analg 1996; 82: 873-4

143. Paul L. Marino: The icu book, third edition 2007; 133-135.

144. Pollock JE, Neal JM, Stephenson CA: Prospective study of the incidence of transient radicular irritation in patients undergoing spinal anesthesia. Anesthesiology 84:1361-1367, 1996.

145. Pinhu L, Whitehead T, Evans T, Griffiths M: ventilator- associated lung injury.The lancet.vol 361 2003;332-340.

146. Prielipp RC, Morell RC, Walker FO, Santos CC, Bennett J, Butterworth J: Ulnar nerve pressure: Influence of arm position and relationship to somatosensory evoked potentials. Anesthesiology 1999; 91: $345-54$

147. Raj P, De Andrés J, Grossi P, Banister R, Sala-Blanch X: Aids to localization of peripheral nerves, Textbook of regional anesthesia. Edited by Raj P. New York, NY, Churchill Livingstone, 2002, pp 251-84

148. Ready L, Plummer M, Haschke R, Austin E, Sumi S: Neurotoxicity of intrathecal local anesthetics in rabbits. Anesthesiology 1985; 63: 364-370

149. Rice A, McMahon S: Peripheral nerve injury caused by injection needles used in regional anaesthesia: Influence of bevel configuration, studied in a rat model. Br J Anaesth 1992; 9: 433-438.

150. Rigler M, Drasner K, Krejcie T: Cauda equina syndrome after continuous spinal anesthesia. Anesth Analg $1991 ; 72 ; 275-81$

151. Rob Toreki : Cannulas. The Glassware Gallery. Interactive Learning Paradigms Incorporated. (1 Dec 2004).

152. Rogawski M, Loscher W: The Neurobiology of antiepileptic drugs. National Review of Neuroscience 2004; 10: 685-692

153. Rosenquist RW, Brown DL: Neuraxial bleeding: fibrinolytics/thrombolytics. Reg Anesth Pain Med 1998; 23: $152-6$.

154. Rousseau J, Reznik M, LeJeune G, Franck G: Sciatic nerve entrapment by pentazocineinduced muscle fibrosis: a case report. Arch Neurol Psychiatry 1979; 36: 723-724

155. Rowbotham M: Mechanisms and Pharmacologic Management of Neuropathic Pain, Peripheral Neuropathy, Fourth Edition. Edited by Dyck P, Thomas P. Philadelphia, Elsevier, 2005, pp 2637-2652

156. Sakura S, Bollen A, Ciriales R, Drasner K: Local anesthetic neurotoxicity does not result from blockade of voltage gated sodium channels. Anesthesia and Analgesia 1995; 81: 338-46.

157. Sala-Blanch X, Pomes J, Matute P, Valls-Sole J, Carrera A, Tomas X: Intraneural injection during anterior approach for sciatic nerve block. Anesthesiology 2004; 101: 1027-1030

158. Saunders K, Louis D, Weingarden S, Watkibus G: Effect of tourniquet time on postoperative quadriceps function. Clin Orthop 1979; 143: 194-199

159. Schneider T, Storz K, Dienemann H, and Hoffmann H: Management of Iatrogenic Tracheobronchial Injuries: A Retrospective Analysis of 29 Cases. Ann. Thorac. Surg., June 1, 2007; 83(6): 1960 - 1964.

160. Schultz A , Ulbing S , Kaider A, Lehofer F : Postdural puncture headache and back pain after spinal anesthesia with 27-gauge Quincke and 26-gauge Ataucan needles . Reg Anesth 1996 ; $21: 461-4$

161. Schurman DA: Ankle-block anesthesia for foot surgery. 1976; 44: 348-352

162. Seddon H: Three types of nerve injury. Brain 1943; 66: 236-288 .

163. Selender D, Dhuner K, Lundborg G: Peripheral nerve injury due to injection needles used for regional anesthesia. An experimental study of the acute effects of needle point trauma. Acta Anaesthesiology Scandinavia 1977; 21: 182-188

164. Selander D, Sjostrand J: Longitudinal spread of intraneurally injected local anesthetics. An experimental study of the initial neural distribution following intraneural injections. Acta Anesth Scand 1978; 22: 622-34

165. Selander D, Edshage S, Wolf T: Paresthesiae or No Paresthesiae? Acta Anaesth Scand 1979; 23: 27-33

166. Selander D, Mansson G, Karlsson L, Svanvik J: Adrenergic vasoconstriction in peripheral nerves of the rabbit. Anesthesiology 1985; 62: 6-10

167. Selander D: Nerve toxicity of local anesthetics, Local Anesthesia and Regional Blockade. Edited by Lofstrom J, Sjostrand U. Amsterdam, Elsevier Science, 1988, pp 77

168. Selander D: Neurotoxicity of local anesthetics: animal data. Reg Anesth 1993; 18: 461-8 
169. Selander D: Peripheral nerve injury after regional anesthesia, Complications of Regional Anesthesia. Edited by Finucane B. Philadelphia, Churchill Livingstone, 1999, pp 105-15.

170. Shah S, Hadzic A, Vloka J, Cafferty M, Moucha C, Santos A: Neurologic complication after anterior sciatic nerve block. Anesth Analg 2005; 100: 1515-7

171. Sharrock N, Savarese J: Anesthesia for orthopedic surgery, Anesthesia, 5th ed Edition. Edited by Miller R, Cucchiara R, Miller E, Reves J, Roizen M, Savarese J. Philadelphia, Churchill Livingstone, 2000, pp 21182139.

172. Sinnott C, Cogswell L, Johnson A: On the mechanism by which epinephrine potentiates lidocaine's peripheral nerve block. Anesthesiology 2003; 98: 181-188

173. Sindrup S, Brosen K, Gram L: The mechanism of action of antidepressants in pain treatment: controlled cross-over studies in diabetic neuropathy. Clinical Neuropharmacology 1992; 15: 380

174. Silvestri E, Martinoli C, Derchi L, Bertolotto M, Chiaramondia M, Rosenberg I: Echotexture of peripheral nerves: Correlation between US and histologic findings to differentiate tendons. Radiology 1995; 197: 291-296

175. Stark R, Wauwatosa W: Neurologic injury from axillary block anesthesia. J Hand Surg 1996; 21A: 391-396

176. Stoelting RK: Postoperative ulnar nerve palsy- Is it a preventable complication? Anesth Analg 76:7-9, 1993.

177. Strasberg J, Atchabahian A, Strasberg S, Watanabe O, Hunter D, Mackinnon S: Peripheral nerve injection injury with antiemetic agents. Journal of Neurotrauma 1999; 16: 99-107

178. Sunderland S: A classification of peripheral nerve injuries producing loss of function. Brain 1951; 74: 491516

179. Sunderland S: Nerve and nerve injury. Edinburgh, Churchill Livingstone, 1978, pp 31-2

180. Sunderland S: The sciatic nerve and its tibial and common peroneal divisions: anatomical and physiological features, Nerves and nerve injuries, 2nd Edition. Edinburgh, Churchill Livingstone, 1978, pp 925-991

181. Taskapan H, Oymak O, Dogukan A, Utas C: Horner's syndrome secondary to internal jugular catheterization. Clin Nephrol. 2001 Jul;56(1):78-80.

182. Timsit JF, Farkas JC, Boyer JM: Central vein catheter-related thrombosis in intensive care patients: incidence, risks factors, and relationship with catheter-related sepsis. Chest, 114:207-13, $1998 \mathrm{Jul}$

183. Tonidandel WT, Mayfield JB: Successful interscalene block with a nerve stimulator may also result after a pectoralis major motor response. Reg Anesth Pain Med 2002; 27: 491-3

184. Tripathi M, Nath S, Gupta RK: Paraplegia after intracord injection during attempted epidural steriod injectin in an awake patient. Anesthe Analg 2005; 101: 1209-11

185. Tsao B, Wilbourn A: Infraclavicular brachial plexus injury following axillary regional block. Muscle \& Nerve 2004; 30: 44-48

186. Tsui B, Armstrong K: Can diredt soinal cord injury occur without paresthesia? A report of delayed spinal cord injury after epidural placement in an awake patient. Anesth Analg 2005; 101: 1212-1214

187. Urmey W, Stanton J: Inability to consistently elicit a motor response following sensory paresthesia during interscalene block administration. Anesthesiology 2002; 96: 552-4

188. Vandermeulen E, Van Aken V , Vermylen J : Anticoagulants and spinal-epidural anesthesia. Anesth Analg $1994 ; 79: 1165-77$

189. Vloka J, Hadzic A, Mulcare R, Lesser J, Koorn R, Thys D: Combined popliteal and posterior cutaneous nerve of the thigh blocks for short saphenous vein stripping in outpatients: an alternative to spinal anesthesia. J Clin Anesth 1997; 9: 618-22

190. Voelckel W, Klima G, Krismer C, Halsonger C, Stadlbauer H, Wenzel V, von Goedecke A: Signs of inflammation after sciatic nerve block in pigs. Anesthesia and Analgesia 2005; 101: 1844-1846

191. Walton J, Fol J, Friedman R, Dorman B: Complete brachial plexus palsy after total shoulder arhtroplasty done with interscalene block anesthesia. Reg Anesth Pain Med 2000; 25: 318-21

192. Warner MA, Warner ME, Martin JT: Ulnar neuropathy. Incidence, outcome, and risk factors in sedated or anesthetized patients. Anesthesiology 1994;81:1332.

193. Warner MA, Warner DO, Harper CM: Lower extremity neuropathies associated with lithotomy positions. Anesthesiology 2000;93:938.

194. Warner MA: Perioperative neuropathies in the lower extremities. In Faust RJ (ed): Anesthesiology Review, 3rd ed. New York, Churchill Livingstone, 2002.

195. Warner ME, Benenfeld SM, Warner MA: Perianesthetic dental injuries. Anesthesiology 1999;90:1302.

196. Warner ME, LaMaster LM, Thoeming AK: Compartment syndrome in surgical patients. Anesthesiology, 94:705-708, 2001. 
197. Watson C, Babul N: Efficacy of oxycodone in neuropathic pain: a randomized trial in postherpatic neuralgia. Neurology 1998; 59: 1837

198. Weaver M, Tandatnick C, Hahn M: Peripheral Nerve Blockade, Regional Anesthesia. Edited by Raj P. Philadelphia, PA, Churchill Livingstone, 2002, pp 857-70

199. 198- Webb R , Kantor G : Obstetrical epidural anaesthesia in a rural Canadian hospital . Can J Anaesth $1992 ; 39: 390-393$

200. 199-Webber S, Jain R: Scalene regional anesthesia for shoulder surgery in a community setting: An assessment of risk. J Bone Joint Surg 2002; 84: 775-779

201. 200-Wedel D, Krohn J, Hall J: Brachial plexus anesthesia in pediatric patients. Mayo Clin Proc 1991; 66: 583-8

202. 201-Weller R, Gerancher J, Crews J, Wade K: Extensive retroperitoneal hematoma without neurologic deficit in two patients who underwent lumbar plexus block and were later anticoagulated. Anesthesiology 2003; 98: 581-585

203. 202-White RK, Morris DM: Diagnosis and management of esophageal perforation. Am Surg 1992; 58: $112-9$.

204. 203-Wylie, Churchill-davidsons : A practice of anesthesia volume $21995 ; 721,739-740,973$.

205. Williams EL, Hart WM Jr, Tempelhoff R: Postoperative ischemic optic neuropathy. Anesth Analg 1995;80:1018.

206. Winnie A: Interscalene brachial plexus block. Anesth Analg 1970; 49: 455-66

207. Wysowski D , Talarico L , Bacsanyi J , Botstein P : Spinal and epidural hematoma and low-molecularweight heparin. (letter) . N Engl J Med $1998 ; 338: 1774$

208. Younes Z, Johnson D: The spectrum of spontaneous and iatrogenic esophageal injury. J Gastroenterol 1999; 29: 306-17. 